\title{
Amino acids and fracture healing
}

Citation for published version (APA):

Meesters, D. M. (2021). Amino acids and fracture healing: Insights on the influence of the argininecitrulline-nitric oxide metabolism during fracture healing and nonunion development. [Doctoral Thesis, Maastricht University]. Maastricht University. https://doi.org/10.26481/dis.20210708dm

Document status and date:

Published: 01/01/2021

DOI:

10.26481/dis.20210708dm

Document Version:

Publisher's PDF, also known as Version of record

\section{Please check the document version of this publication:}

- A submitted manuscript is the version of the article upon submission and before peer-review. There can be important differences between the submitted version and the official published version of record.

People interested in the research are advised to contact the author for the final version of the publication, or visit the DOI to the publisher's website.

- The final author version and the galley proof are versions of the publication after peer review.

- The final published version features the final layout of the paper including the volume, issue and page numbers.

Link to publication

\footnotetext{
General rights rights.

- You may freely distribute the URL identifying the publication in the public portal. please follow below link for the End User Agreement:

www.umlib.nl/taverne-license

Take down policy

If you believe that this document breaches copyright please contact us at:

repository@maastrichtuniversity.nl

providing details and we will investigate your claim.
}

Copyright and moral rights for the publications made accessible in the public portal are retained by the authors and/or other copyright owners and it is a condition of accessing publications that users recognise and abide by the legal requirements associated with these

- Users may download and print one copy of any publication from the public portal for the purpose of private study or research.

- You may not further distribute the material or use it for any profit-making activity or commercial gain

If the publication is distributed under the terms of Article $25 \mathrm{fa}$ of the Dutch Copyright Act, indicated by the "Taverne" license above, 


\section{AMINO ACIDS AND FRACTURE HEALING}

Insights on the influence of the arginine-citrulline-nitric oxide metabolism during fracture healing and nonunion development

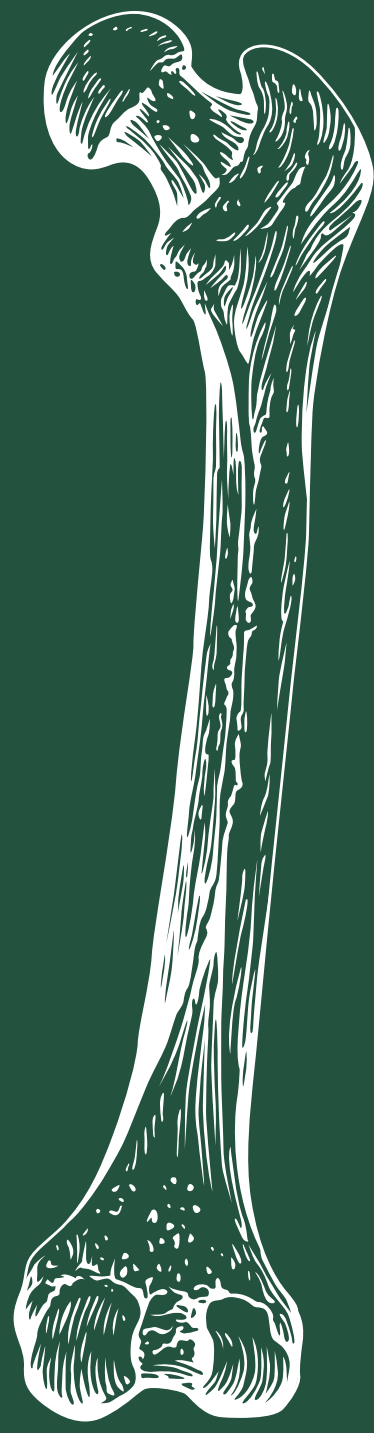

Dennis M. Meesters 



\section{AMINO ACIDS AND FRACTURE HEALING}

Insights on the influence of the arginine-citrullinenitric oxide metabolism during fracture bealing and nonunion development 
(C) Copyright D.M. Meesters, Maastricht 2021

All right reserved. No part of this thesis may be reproduced or transmitted in any form or by any means, electronic or mechanical, including photocopying, recording or any information storage or retrieval system, without written permission from the author.

ISBN: 978-94-6419-242-1

Cover design: Ilse Modder, www.ilsemodder.nl

Layout concept Dennis Meesters

Layout \& design: Ilse Modder, www.ilsemodder.nl

Illustrations: Dennis Meesters

Printed by: $\quad$ Gildeprint Enschede, www.gildeprint.nl

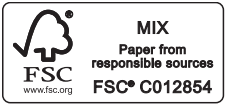

This thesis was supported by: Nederlandse Vereniging voor Traumachirurgie.

The studies presented in this thesis were performed within NUTRIM School of Nutrition and Translational Research in Metabolism 


\title{
AMINO ACIDS AND FRACTURE HEALING
}

\author{
Insights on the influence of the arginine-citrulline- \\ nitric oxide metabolism during fracture bealing \\ and nonunion development
}

\begin{abstract}
PROEFSCHRIFT
ter verkrijging van de graad van doctor aan de Universiteit Maastricht, op gezag van Rector Magnificus, Prof. Dr. Rianne M. Letschert, volgens het besluit van het College van Decanen, in het openbaar te verdedigen op 8 juli 2021 om 14.00 uur
\end{abstract} door

Dennis Maximilian Meesters

Geboren op 28 december 1987 te Heinsberg, Duitsland 


\section{Promotor:}

Prof. Dr. M. Poeze

\section{Copromotor:}

Dr. K.A.P. Wijnands

\section{Beoordelingscommissie:}

Prof. Dr. T.J.M. Welting (voorzitter)

Dr. T.J. Blokhuis

Prof. Dr. M. van Griensven

Prof. Dr. G. Schmidmaier (Universitätsklinikum Heidelberg, Duitsland)

Prof. Dr. M.J. Stoddart (AO Research Institute Davos, Zwitserland) 


\section{TABLE OF CONTENTS}

Chapter $1 \quad$ General introduction 9

Chapter 2 Malnutrition and fracture healing: are specific amino acids important in nonunion development?

Chapter $3 \quad$ Outline of thesis

Chapter 4 Development of a novel murine delayed secondary fracture healing in vivo model using periosteal cauterization

Chapter 5 Deficiency of inducible and endothelial nitric oxide 65 synthase results in diminished bone formation and delayed union and nonunion development

Chapter $6 \quad$ Arginine availability in reamed intramedullary aspirate as predictor of outcome in nonunion healing

Chapter $7 \quad$ Enhancement of fracture healing after citrulline supplementation in mice

Chapter $8 \quad$ General discussion

Chapter 9

Summary

Nederlandse samenvatting

Impact paragraph

List of abbreviations 



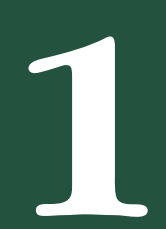

General introduction 


\section{Background in fracture epidemiology}

Injuries due to accidents and violence are one of the major public health burdens, with globally approximately one billion hospital visits and admissions each year ${ }^{1}$. Within The Netherlands, yearly approximately 2 million people visit an emergency department, of which one third (approximately 650,000 patients) have sustained an injury due to an accident, violence or other trauma. These injuries make up 8.3\% of the total health burden and account for approximately 3.6 billion euro in economic costs. Bone fractures are a major part of these injuries (Kerncijfers Letsels 2016, LIS). A fracture is defined as damage to the continuity of the bone, caused by impact, stress or a pathological background. Approximately 250,000 persons who attend the emergency department with an injury have fractured one or more bones during the trauma, resulting in a incidence of fractures of about $1.5 \%$ of the entire Dutch population, which is in accordance with studies within the Western population reporting averages between 11.1 and 11.6 per 1000 persons per year sustaining a fracture in the recent decennia ${ }^{2,3}$. The fracture incidence is expected to rise in the coming decades due to prolonged mobility of the aging population and increasing comorbidities. A gender and age specific incidence is noted. Patients with a fracture below 45 years of age are predominantly male and represent injuries sustained in outdoor activities and motor vehicle accidents, where in the older age group, mainly female patients are found pointing towards an osteoporotic influence and frailty fractures. Comparable differences are found in distribution of fracture location. In patients between 18 and 45 years of age, most commonly found fracture locations are carpus, tibia and ankle/foot fractures. In the elderly patients, mainly radius/ulna and proximal femur or hip fractures are found $^{2}$, which are associated with low energy traumas and fall-related incidents.

\section{Fracture healing}

Osteogenesis (or bone formation) follows two distinct pathways in which new bone is formed by osteoblasts: intramembranous ossification or enchondral ossification. During intramembranous ossification healthy new bone is formed in the medullary cavity of the bone and is mostly found after surgical intervention with plate-screw osteosynthesis to reduce the fracture. In enchondral bone formation, cartilage acts as a precursor for the new bone formation with major influences of the periosteal layer ${ }^{4,5}$. The periosteum is a potent source for osteoblastic progenitor cells and important in blood supply due to its vascularity.

The fracture healing cascade starts directly after trauma and consists of a complex, postnatal, developmental process of four, partially overlapping phases in which there is an elaborate interplay between cells, molecules, growth factors and an extracellular matrix with both anabolic and catabolic responses, ultimately resulting in formation of new bone similar to the pre-fracture situation ${ }^{6}$. The 
different stages of fracture healing follow a definable, temporal sequence:

I. Inflammation;

II. Soft callus formation;

III. Hard callus formation/primary bone formation;

IV. Bone remodelling/secondary bone formation.

Next to damage of to the continuity of the bone, a disruption of the periosteal and endosteal blood supply and other soft tissues around the fracture location will occur due to the injury. The first inflammatory response after sustaining the injury generally follows the principles of standard wound healing. Bleeding at the fracture site will develop into a fracture hematoma which subsequently will attract platelets, macrophages, granulocytes and lymphocytes ${ }^{7}$. Molecular influences during fracture healing are partially comparable with regular wound healing, with upregulation of the angiogenic factor vascular endothelial growth factor (VEGF) and inflammatory markers as interleukins 1 and 6 (IL-1 and IL-6) and inducible nitric oxide synthase. More specifically for fracture healing, bone morphogenetic proteins (BMPs) coordinate and stimulate the cellular response during the first phase of the healing process.

In the second stage of fracture healing, a fibrocartilaginous soft callus will be formed at the fracture site, partially triggered by the mechanical instability leading towards an inflammatory response. Chondrocytes will form cartilage and a generalized fibrous tissue will be produced by fibroblasts which together will replace the granulation tissue with fibrous cartilage connecting the two fracture parts and increasing the stability of the fracture. The proliferation of fibroblasts and chondrocytes in this stage of healing is mainly coordinated by growth factors from the transforming growth factor (TGF- $\beta$ ) family and BMPs, which assist in formation of extracellular matrix proteins (mainly collagen II and X). Due to the presence of pro-angiogenic factors (VEGF, IL-8), capillary in-growth of the soft callus will occur necessary for further healing.

During the third stage, a high osteoblast activity is observed resulting in the formation of a mineralized bone matrix in an irregular woven pattern. Different BMPs are critical key players mediating the de novo primary bone formation, mainly in the differentiation of osteoprogenitors (mainly originating from the periosteum, but also from the circulation and surrounding soft tissues) into osteoblasts. Additionally, mesenchymal cells in the bone marrow will significantly contribute to the bone formation.

After formation of the hard callus, the original shape and structure of the cortical or trabecular bone needs to be restored. Osteoclasts will resorb the irregular woven bone under influence of RANKL (receptor activator of NF- $\kappa \mathrm{B}$ ligand) after which osteoblasts will lay down the lamellar bone resulting in the secondary bone formation. 


\section{Nonunion development}

During the post traumatic healing process, soft tissue and wound healing difficulties might occur which might coincide with infectious complications. The aforementioned factors may additionally contribute to bone related complications such as delayed union and nonunion development.

In 1988, the United States Food and Drug Administration (FDA) defined ${ }^{8}$ nonunion as "established when at least 9 months have elapsed after the initial trauma and without any visible signs of progressive healing for 3 months." Currently, nonunion is defined as a fracture that, in the opinion of the treating physician, has no possibility of healing without any further intervention?. Radiologically, nonunions are characterized by absence of a bridging callus and persisting fracture lines in the cortex with possible pseudoarthrosis formation. The old terminology pseudoarthrosis is presently used for an established nonunion resulting in structural resemblances of a joint. An example of a nonunion s presented in figure 1.1 .
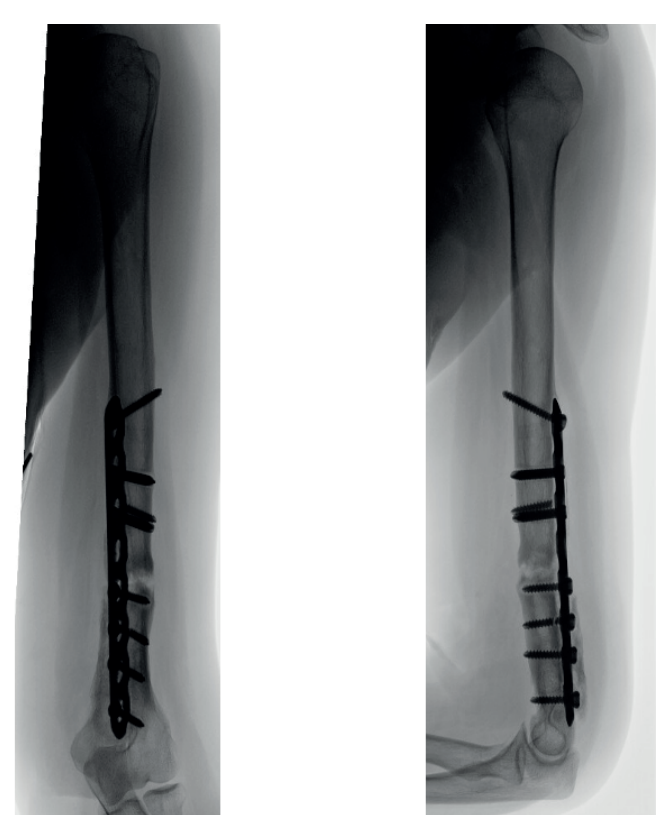

Figure 1.1. Radiographic images of a representative atrophic humerus nonunion in a 63 year old female after plate-screw osteosynthesis.

The incidence of nonunion averages around 5\%10,11 for all types of fractures combined. With fracture incidences describe above, this will lead to a total number between 5000 to 6000 patients with a nonunion each year within the Netherlands. Depending on a wide range of risk factors that negatively influence fracture 
healing resulting in subsequent delayed union or nonunion of the fracture this risk might increase to $46 \%{ }^{12}$. Risk factors contributing to the development of nonunions can be divided into patient dependent and patient independent factors as for instance age and $\operatorname{sex}^{13,14}$.

Age and gender related nonunions mainly follow the same incidence as the fracture incidence with young males that often either have multiple fractures resulting in a cachectic status after long hospitalization or have severe open fractures after major trauma's (motor vehicle accidents) with infectious complications during the healing process. The second peak of nonunion incidence is in older females, mainly as a combination of different comorbidities, diabetes, a poor vascularisation and osteoporosis. Both patient groups may experience nutritional deficiencies contributing to the anabolic disturbances in the patient ${ }^{15,16}$. In children, nonunion development is a rarely seen phenomenon with incidences below $0.2 \%$ in all fractures combined ${ }^{17}$. Other patient dependent risk factors contributing to a diminished bone healing include drug (nicotine, alcohol) and medication, i.e. nonsteroidal anti-inflammatory drugs (NSAIDs) usage. Certain genetic, metabolic or endocrine disorders will also negatively influence the fracture healing process resulting in delayed or nonunions ${ }^{18}$.

Risk factors influencing nonunion development which are patient independent are the degree of soft tissue injury which is related to a higher chance of fracture related infections (possibly resulting in osteomyelitis) and mainly caused by Gram positive Staphylococcus aureus and Staphylococcus epidermidis or the Gram negative microorganisms mainly belonging to the Bacilus, Pseudomonas and Enterobacteriae species ${ }^{19}$. The fracture location also influences the nonunion risk, with tibia fractures (concomitant to open fractures) which are prone to develop nonunions, as well as proximal scaphoid fractures due to vascular disturbance. In long bone fractures of humerus, tibia, and femur, the incidence of nonunion is around 10-15\%. Other patient independent factors increasing the risk for nonunion development are the type of fracture(pattern) and degree of bone loss and subsequent quality of surgical treatment

Nonunion development and its subsequent treatment, usually consisting of improving the stability of the fracture parts and improving bone healing itself by using, for example, bone(marrow) grafting, is accompanied by a major decrease in quality of life with increased periods of revalidation, since nonunion, especially of the long bones, often results in a significant degree of disability if not treated or if treated inadequately. Treatment of nonunions often consists of one or multiple surgical (re)-interventions by iliac crest bone grafting, reamer-irrigator-aspirator treatment or autologous bone marrow grafting, are necessary, before an adequate healing is obtained. Especially in recalcitrant cases, additional growth factors and 
stimuli are needed, possibly by usage of BMP7. Additionally, adequate antibiotic treatment (both type and duration) is of utmost importance in eradicating persisting infections. To achieve an adequate healing after pseudoarthrosis, consensus is that the treatment should adhere to the pentagon approach first described ${ }^{20}$ by Giannoudis and Schmidmaier et al consisting of the five main pillars of adequate treatment:

I. Presence of an adequate scaffold;

II. Growth factors present or able to travel to the fracture site;

III. Sufficient circulatory function;

IV. Mechanical stability of the fracture;

V. A viable bone structure at the fracture location.

The development of nonunion is accompanied by a major decrease in quality of life with increased periods of revalidation, since nonunion, especially of the long bones, often results in a significant degree of disability if not treated ${ }^{21}$. Average costs of nonunion treatment range between $€ 8,000$ and $€$ 90,000, with additional high socio-economic costs due to work disability ${ }^{22,23}$. 


\section{REFERENCES}

1. Haagsma JA, Graetz N, Bolliger I, et al. The global burden of injury: incidence, mortality, disability-adjusted life years and time trends from the Global Burden of Disease study 2013. Inj Prev 2016; 22(1): 3-18.

2. Mills LA, Aitken SA, Simpson A. The risk of nonunion per fracture: current myths and revised figures from a population of over 4 million adults. Acta orthopaedica 2017: 1-6.

3. van Staa TP, Dennison EM, Leufkens HG, Cooper C. Epidemiology of fractures in England and Wales. Bone 2001; 29(6): 517-22.

4. Einhorn TA, Gerstenfeld LC. Fracture healing: mechanisms and interventions. Nat Rev Rheumatol 2015; 11(1): 45-54.

5. Schindeler A, McDonald MM, Bokko P, Little DG. Bone remodeling during fracture repair: The cellular picture. Semin Cell Dev Biol 2008; 19(5): 459-66.

6. Gerstenfeld LC, Cullinane DM, Barnes GL, Graves DT, Einhorn TA. Fracture healing as a postnatal developmental process: molecular, spatial, and temporal aspects of its regulation. J Cell Biochem 2003; 88(5): 873-84.

7. Bastian OW, Mrozek MH, Raaben M, Leenen LPH, Koenderman L, Blokhuis TJ. Serum from the Human Fracture Hematoma Contains a Potent Inducer of Neutrophil Chemotaxis. Inflammation 2018; 41(3): 1084-92.

8. Frolke JP, Patka P. Definition and classification of fracture nonunions. Injury 2007; 38 Suppl 2 S19-22.

9. Calori GM, Mazza EL, Mazzola S, et al. Nonunions. Clin Cases Miner Bone Metab 2017; 14(2): 186-8.

10. Court-Brown CM, Caesar B. Epidemiology of adult fractures: A review. Injury 2006; 37(8): 6917.

11. Zura R, Braid-Forbes MJ, Jeray $\mathrm{K}$, et al. Bone fracture nonunion rate decreases with increasing age: A prospective inception cohort study. Bone 2017; 95: 26-32.

12. Bastian OW, Kuijer A, Koenderman L, et al. Impaired bone healing in multitrauma patients is associated with altered leukocyte kinetics after major trauma. J Inflamm Res 2016; 9: 69-78.

13. Bishop JA, Palanca AA, Bellino MJ, Lowenberg DW. Assessment of compromised fracture healing. J Am Acad Orthop Surg 2012; 20(5): 273-82.

14. Calori GM, Albisetti W, Agus A, Iori S, Tagliabue L. Risk factors contributing to fracture nonunions. Injury 2007; 38 Suppl 2: S11-8.

15. Long CL, Geiger JW, Richards EW, Akin JM, Blakemore WS. Plasma amino acid concentrations in geriatric control and hip-fracture patients. Am J Clin Nutr 1992; 55(6): 1135-41.

16. Wijnands KA, Brink PR, Weijers PH, Dejong CH, Poeze M. Impaired fracture healing associated with amino acid disturbances. Am J Clin Nutr 2012; 95(5): 1270-7.

17. Mills LA, Simpson AH. The risk of nonunion per fracture in children. J Child Orthop 2013; 7(4): $317-22$.

18. Brinker MR, O'Connor DP, Monla YT, Earthman TP. Metabolic and endocrine abnormalities in 
patients with nonunions. J Orthop Trauma 2007; 21(8): 557-70.

19. Neubauer T, Bayer GS, Wagner M. Open fractures and infection. Acta Chir Orthop Traumatol Cech 2006; 73(5): 301-12.

20. Giannoudis PV, Einhorn TA, Schmidmaier G, Marsh D. The diamond concept--open questions. Injury 2008; 39 Suppl 2: S5-8.

21. Schottel PC, O'Connor DP, Brinker MR. Time Trade-Off as a Measure of Health-Related Quality of Life: Long Bone Nonunions Have a Devastating Impact. J Bone Joint Surg Am 2015; 97(17): 1406-10.

22. Kanakaris NK, Giannoudis PV. The health economics of the treatment of long-bone nonunions. Injury 2007; 38 Suppl 2: S77-84.

23. Mills LA, Simpson AH. The relative incidence of fracture nonunion in the Scottish population (5.17 million): a 5-year epidemiological study. BMJ Open 2013; 3(2). 



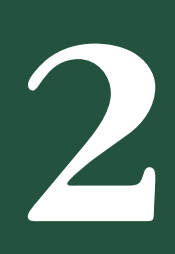

\section{Malnutrition and fracture healing: are specific amino acids important in nonunion development?}

D.M. Meesters
K.A.P. Wijnands
P.R.G. Brink
M. Poeze

Nutrients

2018 


\section{ABSTRACT}

With the increasing incidence of fractures now, and in the future, the absolute number of bone-healing complications such as nonunion development will also increase. Next to fracture-dependent factors such as large bone loss volumes and inadequate stabilization, the nutritional state of these patients is a major influential factor for the fracture repair process. In this review, we will focus on the influence of protein/amino acid malnutrition and its influence on fracture healing. Mainly, the arginine-citrulline-nitric oxide metabolism is of importance since it can affect fracture healing via several precursors of collagen formation, and through nitric oxide synthases it has influences on the bio-molecular inflammatory responses and the local capillary growth and circulation. 


\section{INTRODUCTION}

The absolute number of fractures will increase in the future due to rapid aging of the population and the associated incidence of osteoporosis ${ }^{1}$. Current estimations for a person in the general population on sustaining a fracture are 1 in 100 persons per year ${ }^{2}$, with risks of suffering an osteoporotic fracture ranging between $13 \%$ and $50 \% \%^{3,4}$. The observed change in lifestyle in the older population will lead to people being more active until a higher age and increased sports-related fractures 5 . With increasing incidences in (different types of) fractures, the absolute number of complications during the fracture-healing process, such as nonunion development, will also show an increased prevalence ${ }^{6}$. Next to a drastically diminished quality of life for the patients, high socio-economic costs contribute to the problem of nonunion development ${ }^{7}$. Nutrition has a major influence on fracture healing, with observed fracture-healing impairment in the malnourished and undernourished population. In this review, we will focus on the influence of malnutrition on bio-molecular responses and subsequent complications during the fracture-healing process and nonunion development.

\section{Normal fracture healing}

To understand nonunion development, first normal fracture healing will be discussed. Fracture healing is a complex process of partially overlapping sequential phases, starting with an inflammatory response and hematoma formation caused by the tissue reactions on vascular and soft tissue damage ${ }^{8,9}$. The inflammatory phase is followed by the formation of a soft fibro-cartilaginous matrix consisting primarily out of fibroblasts and chondrocytes, providing primary mechanical stability at the fracture site ${ }^{8}$. The cartilaginous matrix acts as a template for the hard callus in the third stage of the healing process. During the osteogenic phase of primary bone formation, irregular woven bone is formed and vascularization towards the callus increases. Finally, after the primary bone formation, this irregular woven callus is replaced into lamellar bone, thus forming the original cortical and trabecular form of the bone $e^{8-10}$.

\section{Nonunion development}

In the healing process of five to ten percent of all fractures, difficulties occur resulting in or nonunion development of the fracture ${ }^{11-13}$. Although there is no definitive consensus ${ }^{14}$, nonunions are clinically characterized by motion between the fracture parts and a persisting fracture line present on radiographic imaging. This finally results in the development of a synovial pseudo-arthrosis after 6 to 9 months after the initial trauma ${ }^{15,16}$. Traditionally, nonunions are classified either as hypertrophic or atrophic ${ }^{16}$. Hypertrophic nonunions usually are well vascularized 
and exhibit partial callus formation. However, due to the inadequate stabilization of the fracture parts, movement impedes the formation of a solid callus ${ }^{16}$. On the contrary, atrophic nonunions show low levels of callus formation and are usually considered to be of an avascular or metabolic origin hampering callus synthesis. Risk factors for nonunion development are generally divided into fracture dependent or patient dependent ${ }^{17}$. Fracture-dependent factors include a lack of cortical apposition, the presence and degree of communition, displacement of the fracture ${ }^{18}$, blood supply to the fracture region ${ }^{19,20}$, presence of periosteal damage $^{21-23}$ and soft tissue damage associated with infection ${ }^{24}$. Logically, critical sized segmental defects also have a higher nonunion risk due to large segmental bone loss ${ }^{25}$. Patient dependent risk factors include age, gender, genetic disorders, metabolic diseases, smoking, non-steroidal anti-inflammatory drug (NSAID) use and the patient's nutritional status ${ }^{17,22,26}$ among others. As for age and sex, the distribution of nonunions has distinct peaks in males in the age category between 25 and 29 years. As for females, elderly aged around 75 years, where show a sharp incline starting after their 65 th year $^{6}$. This incidence reflects the epidemiology of fractures in men and women and their age distribution ${ }^{27,28}$. Metabolic diseases resulting in vitamin D deficiency, thyroid disorders and parathyroid hormone disorders are more often found in patients who development a nonunion. Medical treatment alone of these comorbidities can result in union of the fracture parts ${ }^{29}$. The nutritional status, especially malnutrition, will be discussed in more detail in the next paragraphs.

\section{PROTEINS AND MALNUTRITION IN FRACTURE HEALING}

\section{Influence of collagens and bone morphogenetic proteins on fracture healing}

Proteins which are most abundant in bone and which have major influences during the fracture-healing process are the different types of collagen and the bone morphogenetic proteins (BMPs).

Collagen is the most abundant protein present in bones ${ }^{30}$. Until now, 28 types of collagen have been discovered, of which type I (Col I) represents $90 \%$ of the total collagen in the human body and which is the main component of the organic part of the bones. A normal type 1 collagen protein consists of so-called alpha-1 type I collagen chains and alpha-2 type 1 collagen chains which combined form a molecule of type I procollagen. Extracellularly, these molecules are processed and arranged into thin fibrils with the ability to cross-link with each other resulting in mature collagen fibers. These mature collagen fibers, three-dimensionally exhibit a triple helical-like structure, which prevents collagen from being broken down by enzymes, contributing to adhesiveness of cells and formation of the extracellular 
matrix $^{31}$. Formation of abnormal and irregular collagen fibers can be the result of a vitamin $\mathrm{C}$ deficiency, since ascorbic acid is a cofactor for collagen synthesis. Presence of irregular fibers will result in a delayed healing of the fracture or possible formation of a decreased strength in the newly formed bone resulting in a higher chance of subsequent fractures ${ }^{30,32}$. In scaffolds which are used for tissue regeneration, collagen is often used since the in vivo stability but also the porelike structure contributes to the adhesion of fibroblasts and osteoblasts ${ }^{33}$.

Other types of collagen which are related to bone healing and bone formation are collagen X (Col X) and collagen XI (Col XI). Both Col X and Col XI are mainly found in (hypertrophic and mineralizing) chondrocytes and so play a role in formation of the soft cartilaginous fibroblastic matrix formed during endochondral ossification.

The second important player are the BMPs. During primary bone formation, BMPs, which are glycoproteins, are involved in mediating the process of osteoblasts synthesizing the mineralized callus ${ }^{34}$. BMPs first described by Marshall Urist in the $1960 s^{35}$ belong to the transforming growth factor (TGF) superfamily ${ }^{36}$. BMPs, are known not only to be active in growth and differentiation but also show high degrees of osteogenic potential in in vitro, as well as in animal and human in vivo research ${ }^{37-40}$. BMP signaling follows a time-dependent sequential cascade of chondrogenesis, osteogenesis, angiogenesis and the synthesis of extracellular matrix $^{41,42}$, allowing them to be used for influencing bone formation throughout the complete course of the fracture-healing process. Although a variety of approximately twenty BMPs have been identified and classified ${ }^{36}$, until now, only recombinant human (rh) BMP2 and rhBMP7 (also known as osteogenic protein-1; OP-1) are used clinically in orthopedic and trauma surgery ${ }^{43,44}$.

Numerous (genetic) studies have found a wide array of signaling pathways leading to different proteins which are involved in the fracture-healing process. One of the most intensively studied is the $\beta$-catenin-dependent Wnt signaling, which has been reviewed extensively before ${ }^{45,46}$. However, since this canonical Wnt signaling pathway mainly is involved in maintaining bone mass, it is mainly investigated within osteoporotic fractures as it might counter the bone volume inhibitory effects of overexpressed molecules as Dickkopf (Dkk) and Sclerostin $(\mathrm{Scl})^{47,48}$, this pathway is not further reviewed within this paper.

\section{Nutritional status and fracture healing}

Older adults ( $>65$ years) are at a higher risk of malnutrition ${ }^{49}$. Malnourishment is the physical condition in which a person's food intake is either too low or high for one or more nutritional factors, or a misbalance between the nutritional factors is present. Associated with malnourishment is a lower body mass index (BMI) which also is correlated with an increased fall risk ${ }^{50}$. This increased fall risk may 
result in a higher risk for sustaining a fracture, hence it may contribute to the total number of nonunions developing in these trauma patients.

A poor nutritional state increases the risk for osteoporotic fractures ${ }^{51}$ and also of nonunion development as osteoporosis leads to a reduction of osteoblasts and callus production ${ }^{22,52}$. Next to osteoporotic fractures, mal/undernourished patients tend to have more fall incidents when compared to non-malnourished patients $^{53}$. The frailty of especially the elderly population undergoing surgery is associated with higher rates of mortality ${ }^{54}$ and a longer hospital stay ${ }^{55}$ and multiple readmissions ${ }^{56,57}$. In addition, the prolonged inactivity of patients after hospital admission and revalidation after a traumatic fracture can result in a substantial loss of skeletal muscle mass of up to $5 \%$ of total muscle mass within the first two weeks after trauma ${ }^{58}$. This sarcopenic state of the patients contributes to the anabolic process of bone formation during the healing period, resulting in a higher chance of nonunion development ${ }^{59,60}$.

A vastly explored method for improving malnourishment is supplementation with different amino acids, which is investigated in animal testing ${ }^{61-64}$ as well as in (hospitalized) patients ${ }^{65-68}$, for a range of different conditions such as cancer, cardiac disease, sepsis, and liver metabolism, but also for its possible beneficial effects on post-surgical infection development ${ }^{69}$ and in orthopedic diseases ${ }^{70}$.

In geriatric trauma patients, the majority of essential and non-essential amino acids is known to be significantly decreased when compared to healthy geriatric control patients ${ }^{71}$. Already in 1976, lower ornithine concentrations were observed in patients with fractures after major trauma ${ }^{72}$ when compared to control patients. These results are in line with the fact that ornithine is, through polyamine production, a precursor for collagen synthesis ${ }^{73}$. Underlining these findings is the fact that a deficiency of ornithine is contributing to the enhanced nonunion risk in multi-trauma, patients ${ }^{17,22}$, which often also exhibit a malnourished state during the prolonged hospitalization and immobilization. Protein-depleted patients with a fracture of the hip showed higher prevalence of complications, a longer admission period in the hospital and a lower one year survival probability ${ }^{74}$ when compared to non-depleted patients.

Next to essential amino acids ${ }^{75,76}$, non-essential amino acids such as glutamine, arginine and their precursors possess beneficial anabolic properties which are essential during fracture healing ${ }^{77}$.

Hughes et al. observed ${ }^{78}$ an enhanced fracture and soft tissue healing, in rats were a closed femoral midshaft fracture was induced with subsequent intramedullary nailing and afterwards received anabolic dietary supplementation, consisting of proteins and the conditionally essential amino acids glutamine, arginine and taurine. Groups with high concentrations of proteins and the conditionally essential amino acids glutamine and arginine (among others) showed increased muscle 
mass and bone mineral density in the fracture callus after a healing period of six weeks when compared with animals that were fed a diet with low concentrations of proteins. In a comparable study ${ }^{79}$, malnourished rats (protein-depleted) which underwent a closed femoral fracture, showed a callus primarily composed of fibrous tissue with decreased periosteal and endosteal callus size and decreased callus strength when compared with control animals which underwent a closed femoral fracture.

The focus in most of these studies ${ }^{75-79}$ is the semi-essential amino acid arginine. During normal physiological conditions, arginine is produced from conversion of citrulline by the two cytosolic enzymes arginosuccinate synthetase (ASS) and arginosuccinate lyase (ASL). The importance of arginine is due to it being the only precursor within the human which physiologically can be converted into nitric oxide (NO). Citrulline exhibits a low dietary intake (13\% of total arginine); however, $60-80 \%$ is contribute by the conversion of glutamine into citrulline in the enterocytes of the small intestine. A third way in which citrulline can be produced is degradation of ornithine via ornithine transcarbamylase. In figure 2.1, a schematic overview of the arginine-citrulline-NO-metabolism is presented.

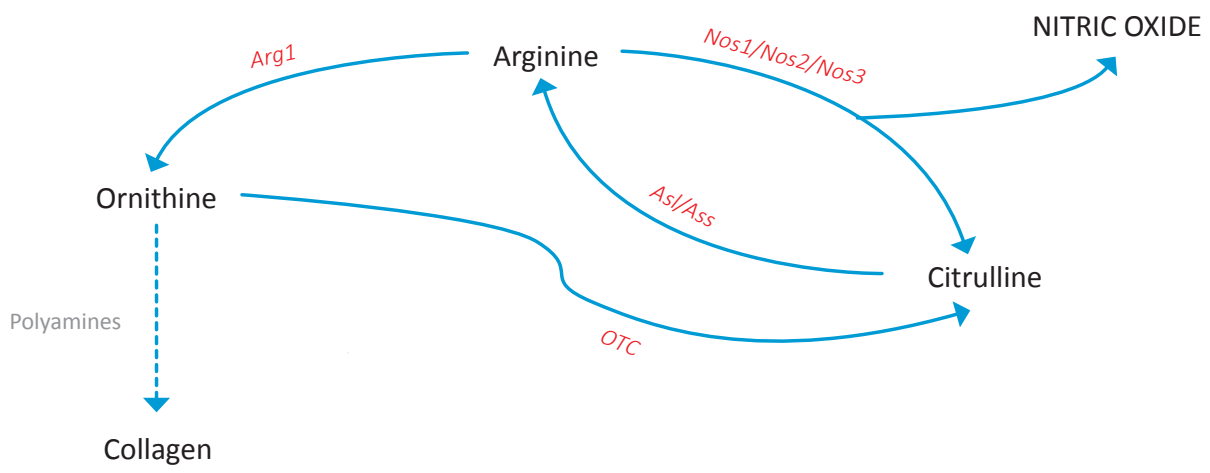

Figure 2.1. Schematic representation of the arginine-citrulline-nitric oxide metabolism. Arginine can be converted into citrulline by one of the isoforms of nitric oxide synthase (NOS1, NOS2 and NOS3). During the conversion, nitric oxide is produced. Citrulline can be converted back into arginine by the enzymes arginosuccinate synthetase (ASS) and arginosuccinate lyase (ASL). Conversion of arginine into ornithine is mainly via arginase 1 (Arg1). Ornithine acts as a precursor for collagen synthesis through conversion via several polyamine molecules. Along a second route, it can be converted into citrulline by the enzyme ornithine transcarbamylase (OTC).

\section{Influences of nitric oxide and nitric oxide synthases on fracture healing}

Several in vitro studies provide a solid scientific base for the possibilities of stimulating the arginine-nitric oxide (NO) metabolism with the interest of enhancing the fracture-healing process. 
Stimulation of human osteoblasts derived from osteopenic patients with arginine has been shown to have a positive effect on proliferation, activation and differentiation and matrix synthesis ${ }^{80}$, thus suggesting possibilities in prevention of osteoporotic fractures.

Previously, Chevalley et al. reported $^{81}$ on the influence of arginine on murine osteoblast-like cells. The supplementation of arginine increased the concentrations of IGF-1 (insulin-like growth factor-1) and the de novo collagen synthesis. As IGF-1 is a potent regulator of osteoblastic bone formation, supplementation of arginine might be a promising option in malnourished patients with osteoporotic fractures $^{82}$. In combination with L-lysine supplementation of arginine, beneficial effects on proliferation were shown in osteoblast cultures from osteopenic rats ${ }^{83}$, resulting in increased type 1 collagen formation.

In 2000, Diwan et al. reported $^{84}$ that nitric oxide had a role in fracture healing as it was expressed in callus tissue during fracture healing. Also, NO was known to play a critical role in other physiological processes such as wound-healing ${ }^{85}$ and tendon healing ${ }^{86}$. Next to its role in fracture repair, NO is known to be involved in a wide range of musculoskeletal conditions such as inflammatory arthritis ${ }^{87,88}$, osteoporosis $^{89}$, aseptic loosening of implanted prosthesis ${ }^{90}$ and tendon healing ${ }^{86,91}$. After inducing a closed right femoral midshaft fracture in rats by three-point bending $^{92}$, the importance and presence of NO production in fracture healing was shown by the activity of calcium-dependent and -independent nitric oxide synthase (NOS1 and NOS3) activity that was detected in homogenized fracture callus by using a conversion assay of $\left[{ }^{3} \mathrm{H}\right]$-arginine to $\left[{ }^{3} \mathrm{H}\right]$-L-citrulline. In addition, immunohistochemical tests localized NOS2 presence in these rats at the junction of fibrous tissue and the cartilage front. On mRNA level, expression of Nos 2 was present after 4, 7 and 15 days of fracture healing, whereas Nos1 and Nos3 were only expressed after 7 and 15 days.

Human fracture callus samples collected in patients undergoing open reduction and internal fixation of a fracture ${ }^{84}$ Nos 2 and Nos3 mRNA was present in 3- to 5-day old fractures. Nos1 was only present in a 90-day old fracture.

Rats fed the nonselective NOS inhibitor L-Nitroso-arginine methyl ester (L-NAME) via the drinking water ad libitum prior to surgery inducing an midshaft femoral fracture and during the follow-up period, showed an approximate 20\% decrease in cross-sectional callus area and average mineral density when compared to rats fed the inactive enantiomer D-NAME ${ }^{84}$. During biomechanical testing, these NOS-inhibited rats showed a decrease in peak failure load, stiffness and energy required to break the healing femur. In addition, supplementation of an NO donor (NONOate derivative of carboxybutyl chitin) resulted in a 30\% increase in crosssectional area was shown when compared to L-NAME treated animals. However, rats which received L-NAME did not show a distinct change in trabecular bone 
formation rate ${ }^{93}$.

These results are in line with studies investigating wound-healing responses, were addition of an NO donor increases skin wound-healing ${ }^{94}$ whereas Nos2 gene deficient animals which showed decreased wound closure rates ${ }^{95}$. In fracture healing, deletion of the Nos 2 gene in mice ${ }^{96}$ showed a significant decrease in maximum energy absorption during biomechanical testing when compared to normal wild type mice. Nos $2^{-/-}$mice receiving NOS2cDNA directly at the fracture site by implantation of a gelatine sponge, showed normal energy absorption and an increased callus cross-sectional area.

In addition to this study, Zhu et al. reported on type specific and time-dependent expression of different NOS isoforms in the fracture-healing process ${ }^{97}$. In an open rat fracture model in which controlled femoral midshaft fracture was made and afterwards fixed with a $1.6 \mathrm{~mm}$ Kirschner wire, all NOS isoforms were expressed during the first 21 days of fracture healing. However, were NOS2 had its peak after 4-7 days, consistent with the inflammatory phase in the fracture-healing process ${ }^{98}$. NOS3 mRNA and protein were mainly expressed between 7-14 days, where osteoblastic differentiation and activity is at is maximum ${ }^{99}$. The neuronal NOS1 was found after 21 days during the remodeling phase of the fracture, indicating a lower importance in nonunion development since disturbances during the early and middle stages of fracture repair generally lead to nonunions.

Next to the distinctive temporal expression of the different NOS isoforms, an isoform dependent spatial localization is found in healing rat fractures ${ }^{100}$. The initially upregulated NOS2 is found mainly along the edge of the periosteal callus, close to the cortical bone and in areas of endochondral ossification. Endothelial NOS3 was primarily present in cells lining blood vessels and cells in the chondral region. Lastly, NOS1 showed a signal between the fibrous tissue and cartilage within the fibrochondral region of the healing fracture ${ }^{97,100}$.

NOS2-derived NO production is important in bone formation by mediating the transduction of a mechanical stimulus into biological responses in bone e3,101,102 $^{\text {. }}$ When treating rats with the selective NOS inhibitor aminoguanidine, bone formation rate, mineral apposition rate and the percentage of mineralizing surface is significantly lower in the proximal tibial epiphysis when compared with control animals ${ }^{93}$. In addition, NOS expression is shown to correlate with new bone formation during distraction osteogenesis in rats ${ }^{103}$.

In a tail-suspension model simulating hindlimb unloading in mice, the role of NOS2 in skeletal adaptation to acute increases was investigated ${ }^{104}$. Gene deficient Nos 2 mice showed a decreased bone volume and bone formation rate after 7 days of tail suspension. During subsequent 14-day reloading, increases in bone formation and volume were abolished in NOS2 mice in comparison with control animals. Treatment with the NO donor nitroglycerine corrected the defective 
responses in NOS2 deficient mice. As presented in our recent study ${ }^{105}$, in a mouse model of delayed fracture healing caused by periosteal cauterization, an absence of NOS2 or NOS3 resulted in a diminished bone formation with significantly lower bone volumes measured and a shift in these mice from delayed union towards nonunion. Comparable results were found in a fracture-healing study conducted by Kdolsky et al., were arginine was administered to guinea-pigs subjected to a $7 \mathrm{~mm}$ diaphyseal and periosteal femoral defect stabilized intramedullary with a Kirschner wire. Radiographic analysis of these animals showed an increased number of healed fractures in the treatment group when compared to control animals ${ }^{62}$.

Osteocalcin, a protein which is produced and secreted by osteoblasts, is increased in serum of rats fed L-NAME with/without addition of L-arginine via the drinking water for a period of 18 days. Serum levels reflect systemic bone formation; however, bone formation indices in tibial epiphysis in these rats showed no correlation with osteocalcin concentrations ${ }^{93}$.

\section{Possible applications for D-enantiomeric amino acids}

In recent years, a possible role for D-amino acids had been investigated in bone research and fracture repair ${ }^{106,107}$. With the development of different fracture-healing animal models ${ }^{108}$, mainly in mice and rats, the possibilities for research into infectious complications during bone healing have increased ${ }^{109-111}$. In the clinical situation there is still a high risk for developing implant-related infectious complications ${ }^{112}$. Staphylococcus aureus is the micro-organism which is most abundant in chronic osteomyelitis ${ }^{113}$ and with a high potential of forming biofilms ${ }^{114}$ and associated incidences of nonunion development ${ }^{115}$. Recent studies by Sanchez et al. showed that a local delivery of a combination of D-amino acids from biofilm-dispersive scaffolds showed a reduced $S$. aureus contamination in vivo and in vitro ${ }^{107}$. On the contrary, although in vitro experiments showed that D-amino acids also inhibit bone marrow stromal cell proliferation and differentiation of osteoblasts and osteoclasts, new bone formation in an ovine model is not hampered ${ }^{106}$. More research into the role of D-amino acids needs to be conducted to elucidate the so far contradicting results found in these studies.

\section{CONCLUDING REMARKS AND FUTURE POSSIBILITIES}

In this review, a large amount of preclinical evidence is presented to substantiate the hypothesis that in clinical development of nonunion, specific amino acid deficiencies play an important role. Mainly, the arginine-citrulline-nitric oxide metabolism has a major influence on the process of fracture repair, more 
specifically appropriate concentrations of amino acids and temporal expression of nitric oxide synthase enzymes are of utmost importance for an adequate bone-healing process. Future clinical research should focus on detecting specific amino acid deficiencies in patients directly after sustaining a fracture, and on the other hand on randomized controlled trials focusing on the results of amino acid supplementation in patients with observed deficiencies. 


\section{REFERENCES}

1. Kannus P, Niemi S, Palvanen M, et al. Continuously rising problem of osteoporotic knee fractures in elderly women: nationwide statistics in Finland in 1970-1999 and predictions until the year 2030. Bone 2001; 29(5): 419-23.

2. van Staa TP, Dennison EM, Leufkens HG, Cooper C. Epidemiology of fractures in England and Wales. Bone 2001; 29(6): 517-22.

3. Alves CJ, Neto E, Sousa DM, et al. Fracture pain-Traveling unknown pathways. Bone 2016; 85: 107-14.

4. Johnell O, Kanis J. Epidemiology of osteoporotic fractures. Osteoporosis international : a journal established as result of cooperation between the European Foundation for Osteoporosis and the National Osteoporosis Foundation of the USA 2005; 16 Suppl 2: S3-7.

5. Knuth AG, Hallal PC. Temporal trends in physical activity: a systematic review. Journal of physical activity \& bealth 2009; 6(5): 548-59.

6. Mills LA, Simpson AH. The relative incidence of fracture non-union in the Scottish population (5.17 million): a 5-year epidemiological study. BMJ Open 2013; 3(2).

7. Antonova E, Le TK, Burge R, Mershon J. Tibia shaft fractures: costly burden of nonunions. BMC musculoskeletal disorders 2013; 14: 42.

8. Gerstenfeld LC, Cullinane DM, Barnes GL, Graves DT, Einhorn TA. Fracture healing as a postnatal developmental process: molecular, spatial, and temporal aspects of its regulation. J Cell Biochem 2003; 88(5): 873-84.

9. Schindeler A, McDonald MM, Bokko P, Little DG. Bone remodeling during fracture repair: The cellular picture. Semin Cell Dev Biol 2008; 19(5): 459-66.

10. Claes L, Recknagel S, Ignatius A. Fracture healing under healthy and inflammatory conditions. Nat Rev Rheumatol 2012; 8(3): 133-43.

11. Court-Brown CM, McQueen MM. Nonunions of the proximal humerus: their prevalence and functional outcome. The Journal of trauma 2008; 64(6): 1517-21.

12. Einhorn TA. Enhancement of fracture-healing. J Bone Joint Surg Am 1995; 77(6): 940-56.

13. Wijnands KA, Brink PR, Weijers PH, Dejong CH, Poeze M. Impaired fracture healing associated with amino acid disturbances. Am J Clin Nutr 2012; 95(5): 1270-7.

14. Bhandari M, Guyatt GH, Swiontkowski MF, Tornetta P, 3rd, Sprague S, Schemitsch EH. A lack of consensus in the assessment of fracture healing among orthopaedic surgeons. Journal of orthopaedic trauma 2002; 16(8): 562-6.

15. Frolke JP, Patka P. Definition and classification of fracture non-unions. Injury 2007; 38 Suppl 2: S19-22.

16. Megas P. Classification of non-union. Injury 2005; 36 Suppl 4: S30-7.

17. Bishop JA, Palanca AA, Bellino MJ, Lowenberg DW. Assessment of compromised fracture healing. J Am Acad Orthop Surg 2012; 20(5): 273-82.

18. Robinson CM, Court-Brown CM, McQueen MM, Wakefield AE. Estimating the risk of nonunion following nonoperative treatment of a clavicular fracture. J Bone Joint Surg Am 2004; 86-A(7): 
1359-65.

19. Kozin SH. Incidence, mechanism, and natural history of scaphoid fractures. Hand Clin 2001; 17(4): 515-24.

20. Rosenberg GA, Sferra JJ. Treatment strategies for acute fractures and nonunions of the proximal fifth metatarsal. The Journal of the American Academy of Orthopaedic Surgeons 2000; 8(5): 332-8.

21. Allen MR, Hock JM, Burr DB. Periosteum: biology, regulation, and response to osteoporosis therapies. Bone 2004; 35(5): 1003-12.

22. Calori GM, Albisetti W, Agus A, Iori S, Tagliabue L. Risk factors contributing to fracture nonunions. Injury 2007; 38 Suppl 2: S11-8.

23. Roberts SJ, van Gastel N, Carmeliet G, Luyten FP. Uncovering the periosteum for skeletal regeneration: The stem cell that lies beneath. Bone 2015; 70C: 10-8.

24. Gustilo RB, Gruninger RP, Davis T. Classification of type III (severe) open fractures relative to treatment and results. Orthopedics 1987; 10(12): 1781-8.

25. Schmidmaier G, Capanna R, Wildemann B, Beque T, Lowenberg D. Bone morphogenetic proteins in critical-size bone defects: what are the options? Injury 2009; 40 Suppl 3: S39-43.

26. Hernandez RK, Do TP, Critchlow CW, Dent RE, Jick SS. Patient-related risk factors for fracturehealing complications in the United Kingdom General Practice Research Database. Acta orthopaedica 2012; 83(6): 653-60.

27. Buhr AJ, Cooke AM. Fracture patterns. Lancet 1959; 1(7072): 531-6.

28. Court-Brown CM, Caesar B. Epidemiology of adult fractures: A review. Injury 2006; 37(8): 691-7.

29. Brinker MR, O’Connor DP, Monla YT, Earthman TP. Metabolic and endocrine abnormalities in patients with nonunions. J Orthop Trauma 2007; 21(8): 557-70.

30. MacKay D, Miller AL. Nutritional support for wound healing. Altern Med Rev 2003; 8(4): 359-77.

31. Cunniffe GM, Dickson GR, Partap S, Stanton KT, O'Brien FJ. Development and characterisation of a collagen nano-hydroxyapatite composite scaffold for bone tissue engineering. JMater Sci Mater Med 2010; 21(8): 2293-8.

32. Gross RL. The effect of ascorbate on wound healing. Int Ophthalmol Clin 2000; 40(4): 51-7.

33. Oliveira SM, Ringshia RA, Legeros RZ, et al. An improved collagen scaffold for skeletal regeneration. J Biomed Mater Res A 2010; 94(2): 371-9.

34. Nakase T, Yoshikawa H. Potential roles of bone morphogenetic proteins (BMPs) in skeletal repair and regeneration. J Bone Miner Metab 2006; 24(6): 425-33.

35. Urist MR. Bone: formation by autoinduction. Science 1965; 150(3698): 893-9.

36. Axelrad TW, Einhorn TA. Bone morphogenetic proteins in orthopaedic surgery. Cytokine Growth Factor Rev 2009; 20(5-6): 481-8.

37. Barnes GL, Kostenuik PJ, Gerstenfeld LC, Einhorn TA. Growth factor regulation of fracture repair. J Bone Miner Res 1999; 14(11): 1805-15.

38. Cheng H, Jiang W, Phillips FM, et al. Osteogenic activity of the fourteen types of human bone morphogenetic proteins (BMPs). The Journal of bone and joint surgery American volume 2003; 85-A(8): 1544-52.

39. Cho TJ, Gerstenfeld LC, Einhorn TA. Differential temporal expression of members of the 
transforming growth factor beta superfamily during murine fracture healing. Journal of bone and mineral research : the official journal of the American Society for Bone and Mineral Research 2002; 17(3): 513-20

40. Kloen P, Di Paola M, Borens O, et al. BMP signaling components are expressed in human fracture callus. Bone 2003; 33(3): 362-71.

41. Bustos-Valenzuela JC, Halcsik E, Bassi EJ, Demasi MA, Granjeiro JM, Sogayar MC. Expression, purification, bioactivity, and partial characterization of a recombinant human bone morphogenetic protein-7 produced in human 293T cells. Molecular biotechnology 2010; 46(2): 118-26.

42. Carreira AC, Lojudice FH, Halcsik E, Navarro RD, Sogayar MC, Granjeiro JM. Bone morphogenetic proteins: facts, challenges, and future perspectives. Journal of dental research 2014; 93(4): 33545 .

43. Calori GM, Mazza E, Colombo M, Ripamonti C, Tagliabue L. Treatment of long bone non-unions with polytherapy: indications and clinical results. Injury 2011; 42(6): 587-90.

44. Giannoudis PV, Gudipati S, Harwood P, Kanakaris NK. Long bone non-unions treated with the diamond concept: a case series of 64 patients. Injury 2015; 46 Suppl 8: S48-54.

45. Secreto FJ, Hoeppner LH, Westendorf JJ. Wnt signaling during fracture repair. Curr Osteoporos Rep 2009; 7(2): 64-9.

46. Westendorf JJ, Kahler RA, Schroeder TM. Wnt signaling in osteoblasts and bone diseases. Gene 2004; 341: 19-39.

47. Ali A, Hoeflich KP, Woodgett JR. Glycogen synthase kinase-3: properties, functions, and regulation. Chem Rev 2001; 101(8): 2527-40

48. Bao Q, Chen S, Qin H, et al. An appropriate Wnt/beta-catenin expression level during the remodeling phase is required for improved bone fracture healing in mice. Sci Rep 2017; 7(1): 2695.

49. Deutz NE, Matheson EM, Matarese LE, et al. Readmission and mortality in malnourished, older, hospitalized adults treated with a specialized oral nutritional supplement: A randomized clinical trial. Clinical nutrition 2016; 35(1): 18-26.

50. Koski K, Luukinen H, Laippala P, Kivela SL. Risk factors for major injurious falls among the homedwelling elderly by functional abilities. A prospective population-based study. Gerontology 1998; 44(4): 232-8.

51. Cederholm T, Hedstrom M. Nutritional treatment of bone fracture. Current opinion in clinical nutrition and metabolic care 2005; 8(4): 377-81.

52. Lill CA, Hesseln J, Schlegel U, Eckhardt C, Goldhahn J, Schneider E. Biomechanical evaluation of healing in a non-critical defect in a large animal model of osteoporosis. Journal of orthopaedic research : official publication of the Orthopaedic Research Society 2003; 21(5): 836-42.

53. Neyens J, Halfens R, Spreeuwenberg M, et al. Malnutrition is associated with an increased risk of falls and impaired activity in elderly patients in Dutch residential long-term care (LTC): a crosssectional study. Arch Gerontol Geriatr 2013; 56(1): 265-9.

54. Lim SL, Ong KC, Chan YH, Loke WC, Ferguson M, Daniels L. Malnutrition and its impact on cost of hospitalization, length of stay, readmission and 3-year mortality. Clinical nutrition 2012; 
31(3): $345-50$.

55. Oakland K, Nadler R, Cresswell L, Jackson D, Coughlin PA. Systematic review and meta-analysis of the association between frailty and outcome in surgical patients. Annals of the Royal College of Surgeons of England 2016; $98(2): 80-5$.

56. Allard JP, Keller H, Jeejeebhoy KN, et al. Malnutrition at Hospital Admission-Contributors and Effect on Length of Stay: A Prospective Cohort Study From the Canadian Malnutrition Task Force. JPEN Journal of parenteral and enteral nutrition 2015.

57. Sullivan DH, Sun S, Walls RC. Protein-energy undernutrition among elderly hospitalized patients: a prospective study. JAMA 1999; 281(21): 2013-9.

58. Wall BT, Dirks ML, Snijders T, Senden JM, Dolmans J, van Loon LJ. Substantial skeletal muscle loss occurs during only 5 days of disuse. Acta Physiol (Oxf) 2014; 210(3): 600-11.

59. Cawthon PM, Blackwell TL, Cauley J, et al. Evaluation of the Usefulness of Consensus Definitions of Sarcopenia in Older Men: Results from the Observational Osteoporotic Fractures in Men Cohort Study. J Am Geriatr Soc 2015; 63(11): 2247-59.

60. Zura R, Braid-Forbes MJ, Jeray K, et al. Bone fracture nonunion rate decreases with increasing age: A prospective inception cohort study. Bone 2017; 95: 26-32.

61. Angele MK, Fitzal F, Smail N, et al. L-arginine attenuates trauma-hemorrhage-induced liver injury. Crit Care Med 2000; 28(9): 3242-8.

62. Kdolsky RK, Mohr W, Savidis-Dacho H, et al. The influence of oral L-arginine on fracture healing: an animal study. Wien Klin Wochenschr 2005; 117(19-20): 693-701.

63. Poeze M, Bruins MJ, Kessels F, Luiking YC, Lamers WH, Deutz NE. Effects of L-arginine pretreatment on nitric oxide metabolism and hepatosplanchnic perfusion during porcine endotoxemia. $A m J$ Clin Nutr 2011; 93(6): 1237-47.

64. Wijnands KA, Vink H, Briede JJ, et al. Citrulline a More Suitable Substrate than Arginine to Restore NO Production and the Microcirculation during Endotoxemia. PLoS One 2012; 7(5): e37439.

65. Jones MS, Rivera M, Puccinelli CL, Wang MY, Williams SJ, Barber AE. Targeted amino acid supplementation in diabetic foot wounds: pilot data and a review of the literature. Surg Infect (Larchmt) 2014; 15(6): 708-12

66. Luiking YC, Poeze M, Deutz NE. Arginine infusion in patients with septic shock increases nitric oxide production without haemodynamic instability. Clinical science 2015; 128(1): 57-67.

67. Luiking YC, Poeze M, Ramsay G, Deutz NE. Reduced citrulline production in sepsis is related to diminished de novo arginine and nitric oxide production. Am J Clin Nutr 2009; 89(1): 142-52.

68. van Wijck K, Wijnands KA, Meesters DM, et al. L-citrulline improves splanchnic perfusion and reduces gut injury during exercise. Medicine and science in sports and exercise 2014; 46(11): 2039-46.

69. Daly JM, Reynolds J, Thom A, et al. Immune and metabolic effects of arginine in the surgical patient. Annals of surgery 1988; 208(4): 512-23.

70. Evans CH, Stefanovic-Racic M, Lancaster J. Nitric oxide and its role in orthopaedic disease. Clinical orthopaedics and related research 1995; (312): 275-94.

71. Long CL, Geiger JW, Richards EW, Akin JM, Blakemore WS. Plasma amino acid concentrations in 
geriatric control and hip-fracture patients. Am J Clin Nutr 1992; 55(6): 1135-41.

72. Woolf LI, Grovers AC, Moore JP, Duff JH, Finley RJ, Loomer RL. Arterial plasma amino acids in patients with serious postoperative infection and in patients with major fractures. Surgery 1976; 79(3): 283-92

73. Vittur F, Lunazzi G, Moro L, et al. A possible role for polyamines in cartilage in the mechanism of calcification. Biochim Biophys Acta 1986; 881(1): 38-45.

74. Patterson BM, Cornell CN, Carbone B, Levine B, Chapman D. Protein depletion and metabolic stress in elderly patients who have a fracture of the hip. J Bone Joint Surg Am 1992; 74(2): 251-60.

75. Fini M, Aldini NN, Cane V, et al. Effects of essential amino acids and lactose on bony fractures and defects in rabbits: a preliminary histomorphometric study. Arch Orthop Trauma Surg 1999; 119(1-2): 39-45.

76. Volpi E, Kobayashi H, Sheffield-Moore M, Mittendorfer B, Wolfe RR. Essential amino acids are primarily responsible for the amino acid stimulation of muscle protein anabolism in healthy elderly adults. Am J Clin Nutr 2003; 78(2): 250-8.

77. De Bandt JP, Cynober LA. Amino acids with anabolic properties. Curr Opin Clin Nutr Metab Care 1998; 1(3): 263-72.

78. Hughes MS, Kazmier P, Burd TA, et al. Enhanced fracture and soft-tissue healing by means of anabolic dietary supplementation. J Bone Joint Surg Am 2006; 88(11): 2386-94.

79. Day SM, DeHeer DH. Reversal of the detrimental effects of chronic protein malnutrition on long bone fracture healing. J Orthop Trauma 2001; 15(1): 47-53.

80. Torricelli P, Fini M, Giavaresi G, Giardino R. Human osteopenic bone-derived osteoblasts: essential amino acids treatment effects. Artif Cells Blood Substit Immobil Biotechnol 2003; 31(1): $35-46$.

81. Chevalley T, Rizzoli R, Manen D, Caverzasio J, Bonjour JP. Arginine increases insulin-like growth factor-I production and collagen synthesis in osteoblast-like cells. Bone 1998; 23(2): 103-9.

82. Trippel SB. Potential role of insulinlike growth factors in fracture healing. Clinical orthopaedics and related research 1998; (355 Suppl): S301-13

83. Fini M, Torricelli P, Giavaresi G, Carpi A, Nicolini A, Giardino R. Effect of L-lysine and L-arginine on primary osteoblast cultures from normal and osteopenic rats. Biomed Pharmacother 2001; 55(4): 213-20.

84. Diwan AD, Wang MX, Jang D, Zhu W, Murrell GA. Nitric oxide modulates fracture healing. J Bone Miner Res 2000; 15(2): 342-51.

85. Schaffer MR, Tantry U, Gross SS, Wasserburg HL, Barbul A. Nitric oxide regulates wound healing. J Surg Res 1996; 63(1): 237-40.

86. Murrell GA, Szabo C, Hannafin JA, et al. Modulation of tendon healing by nitric oxide. Inflamm Res 1997; 46(1): 19-27.

87. Sakurai H, Kohsaka H, Liu MF, et al. Nitric oxide production and inducible nitric oxide synthase expression in inflammatory arthritides. J Clin Invest 1995; 96(5): 2357-63.

88. Stefanovic-Racic M, Meyers K, Meschter C, Coffey JW, Hoffman RA, Evans CH. N-monomethyl arginine, an inhibitor of nitric oxide synthase, suppresses the development of adjuvant arthritis 
in rats. Arthritis Rheum 1994; 37(7): 1062-9.

89. Wimalawansa SJ, De Marco G, Gangula P, Yallampalli C. Nitric oxide donor alleviates ovariectomyinduced bone loss. Bone 1996; 18(4): 301-4.

90. Hukkanen M, Corbett SA, Batten J, et al. Aseptic loosening of total hip replacement. Macrophage expression of inducible nitric oxide synthase and cyclo-oxygenase-2, together with peroxynitrite formation, as a possible mechanism for early prosthesis failure. J Bone Joint Surg Br 1997; 79(3): 467-74.

91. Xia W, Szomor Z, Wang Y, Murrell GA. Nitric oxide enhances collagen synthesis in cultured human tendon cells. Journal of orthopaedic research : official publication of the Orthopaedic Research Society 2006; 24(2): 159-72.

92. Ekeland A, Engesaeter LB, Langeland N. Mechanical properties of fractured and intact rat femora evaluated by bending, torsional and tensile tests. Acta Orthop Scand 1981; 52(6): 605-13.

93. Turner CH, Owan I, Jacob DS, McClintock R, Peacock M. Effects of nitric oxide synthase inhibitors on bone formation in rats. Bone 1997; 21(6): 487-90.

94. Shabani M, Pulfer SK, Bulgrin JP, Smith DJ. Enhancement of wound repair with a topically applied nitric oxide-releasing polymer. Wound Repair Regen 1996; 4(3): 353-62.

95. Yamasaki K, Edington HD, McClosky C, et al. Reversal of impaired wound repair in iNOS-deficient mice by topical adenoviral-mediated iNOS gene transfer. The Journal of clinical investigation 1998; 101(5): 967-71.

96. Baldik Y, Diwan AD, Appleyard RC, Fang ZM, Wang Y, Murrell GA. Deletion of iNOS gene impairs mouse fracture healing. Bone 2005; 37(1): 32-6.

97. Zhu W, Diwan AD, Lin JH, Murrell GA. Nitric oxide synthase isoforms during fracture healing. $J$ Bone Miner Res 2001; 16(3): 535-40.

98. Einhorn TA, Majeska RJ, Rush EB, Levine PM, Horowitz MC. The expression of cytokine activity by fracture callus. J Bone Miner Res 1995; 10(8): 1272-81.

99. Hikiji H, Shin WS, Oida S, Takato T, Koizumi T, Toyo-oka T. Direct action of nitric oxide on osteoblastic differentiation. FEBS Lett 1997; 410(2-3): 238-42.

100. Zhu W, Murrell GA, Lin J, Gardiner EM, Diwan AD. Localization of nitric oxide synthases during fracture healing. J Bone Miner Res 2002; 17(8): 1470-7.

101. Pilla AA. Nonthermal electromagnetic fields: from first messenger to therapeutic applications. Electromagn Biol Med 2013; 32(2): 123-36.

102. Turner CH, Takano Y, Owan I, Murrell GA. Nitric oxide inhibitor L-NAME suppresses mechanically induced bone formation in rats. Am J Physiol 1996; 270(4 Pt 1): E634-9.

103. de Albuquerque RF, Jr., Aparecida Del Bel E, Brentegani LG, Moura de Oliveira MT, Mardegan Issa JP. Trigeminal nitric oxide synthase expression correlates with new bone formation during distraction osteogenesis. Calcified tissue international 2008; 82(4): 309-15.

104. Watanuki M, Sakai A, Sakata T, et al. Role of inducible nitric oxide synthase in skeletal adaptation to acute increases in mechanical loading. J Bone Miner Res 2002; 17(6): 1015-25.

105. Meesters DM, Neubert S, Wijnands KA, et al. Deficiency of inducible and endothelial nitric oxide synthase results in diminished bone formation and delayed union and nonunion development. 
Bone 2016; 83: 111-8.

106. Harmata AJ, Ma Y, Sanchez CJ, et al. D-amino acid inhibits biofilm but not new bone formation in an ovine model. Clinical orthopaedics and related research 2015; 473(12): 3951-61.

107. Sanchez CJ, Jr., Prieto EM, Krueger CA, et al. Effects of local delivery of D-amino acids from biofilm-dispersive scaffolds on infection in contaminated rat segmental defects. Biomaterials 2013; 34(30): 7533-43.

108. Holstein JH, Garcia P, Histing T, et al. Advances in the establishment of defined mouse models for the study of fracture healing and bone regeneration. J Orthop Trauma 2009; 23(5 Suppl): S31-8.

109. Inzana JA, Schwarz EM, Kates SL, Awad HA. A novel murine model of established Staphylococcal bone infection in the presence of a fracture fixation plate to study therapies utilizing antibioticladen spacers after revision surgery. Bone 2015; 72: 128-36.

110. Li D, Gromov K, Soballe K, et al. Quantitative mouse model of implant-associated osteomyelitis and the kinetics of microbial growth, osteolysis, and humoral immunity. Journal of orthopaedic research: official publication of the Orthopaedic Research Society 2008; 26(1): 96-105.

111. Reizner W, Hunter JG, O'Malley NT, Southgate RD, Schwarz EM, Kates SL. A systematic review of animal models for Staphylococcus aureus osteomyelitis. European cells \& materials 2014; 27: 196-212.

112. Darouiche RO. Treatment of infections associated with surgical implants. The New England journal of medicine 2004; 350(14): 1422-9.

113. Brady RA, Leid JG, Calhoun JH, Costerton JW, Shirtliff ME. Osteomyelitis and the role of biofilms in chronic infection. FEMS immunology and medical microbiology 2008; 52(1): 13-22.

114. Costerton JW, Montanaro L, Arciola CR. Biofilm in implant infections: its production and regulation. Int J Artif Organs 2005; 28(11): 1062-8.

115. Palmer M, Costerton W, Sewecke J, Altman D. Molecular techniques to detect biofilm bacteria in long bone nonunion: a case report. Clinical orthopaedics and related research 2011; 469(11): 3037-42. 



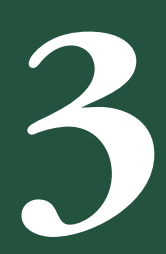

Outline of thesis 
The main hypothesis of this thesis is that the arginine-citrulline-nitric oxide metabolism has a key influence on the fracture healing process and disturbances in the arginine substrate metabolism play an essential role in development of fracture nonunion. By improving or stimulating this substrate metabolism, fracture healing can be enhanced and nonunion formation diminished.

The research objectives which were formulated to investigate this hypothesis were:

I. To develop a reliable animal model which can be used to investigate the effect of metabolic disturbances on fracture healing, delayed union and nonunion development;

II. To investigate the results on fracture healing of a deficient argininecitrulline-nitric oxide metabolism;

III. To improve knowledge on risk factors contributing to a successful or failed treatment for nonunions and assess the link between treatment success and arginine availability;

IV. To study the fracture healing process after stimulation of the argininecitrulline-nitric oxide metabolism.

To properly indicate the different results obtained studying the abovementioned research objectives, this thesis is divided into several chapters. Chapter 1 starts with a general introduction on fracture healing and nonunion development. This if followed by chapter 2 with a literature review on influences of malnutrition, specifically amino acid deficiencies on fracture healing and nonunion formation. In chapter 3, the general outline of this thesis is presented.

After the introductory chapters, the development of a reliable delayed union and nonunion mouse model using periosteal cauterization is presented in chapter 4. The influence of nitric oxide synthase deficiencies disturbing the argininecitrulline-nitric oxide metabolism and influencing fracture repair using the described model is studied in chapter $\mathbf{5}$.

Investigations into the link between successful or failed treatment for nonunions and the argininie availability in reamed intramedullary bone marrow aspirate is shown in chapter 6.

Chapter 7 of this thesis shows the positive results obtained of stimulating the substrate metabolism in a fracture healing model in mice.

Finally, a general discussion on and summary of all results obtained in the studies on nonunion development and influences of arginine, citrulline and nitric oxide 
presented in this thesis is shown in chapter $\mathbf{8}$ with recommendations for further research in the future. Chapter 9 consists of an English and Dutch summary of the work presented in this thesis along with a paragraph describing the scientific and socio-economic impact of the results obtained in this thesis. In chapter 10, information about the author is presented. 



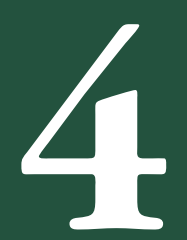

\title{
Development of a novel murine delayed secondary fracture healing in vivo model using periosteal cauterization
}

\author{
I. Gröngröft* \\ S. Wissing* \\ D.M. Meesters \\ M. Poeze \\ R. Matthys-Mark \\ K. Ito \\ S. Zeiter
}

Archives of Orthopaedic and Trauma Surgery

2019

"Ina Gröngröft and Sandra Wissing shared first authorship 


\section{ABSTRACT}

\section{Background}

Delayed union and nonunion development remains a major clinical problematic complication during fracture healing, with partially unclear pathophysiology. Incidences range from $5 \%$ to $40 \%$ in high risk patients, such as patients with periosteal damage. The periosteum is essential in adequate fracture healing, especially during soft callus formation. In this study, we hypothesize that inducing periosteal damage in a murine bone healing model will result in a novel delayed union model.

\section{Methods}

A mid-shaft femoral non-critically sized osteotomy was created in skeletally mature $\mathrm{C} 57 \mathrm{BL} / 6$ mice and stabilized with a bridging plate. In half of the mice, a thin band of periosteum adjacent to the osteotomy was cauterized. Over 42 days of healing, radiographic, biomechanical, micro-computed tomography and histological analysis was performed to assess the degree of fracture healing.

\section{Results}

Analysis showed complete secondary fracture healing in the control group without periosteal injury. Whereas the periosteal injury group demonstrated less than half as much maximum callus volume $(\mathrm{p}<0.05)$ and bridging, recovery of stiffness and temporal expression of callus growth and remodelling was delayed by 7 to 15 days.

\section{Conclusion}

This paper introduces a novel mouse model of delayed union without a critically sized defect and with standardized biomechanical conditions, which enables further investigation into the molecular biological, biomechanical and biochemical processes involved in (delayed) fracture healing and nonunion development. This model provides a continuum between normal fracture healing and the development of nonunions. 


\section{INTRODUCTION}

Delayed or complete failure of fracture healing remains a problematic complication during fracture healing, with general incidences ranging between 5\% and 10\%1,2 and up to date, the pathophysiologic mechanisms for delayed fracture healing are not completely elucidated ${ }^{3}$.

During the last decade(s), in vivo research in rodents has resulted in a wide range of different animal models for fracture healing and compromised healing resulting in delayed union and nonunion development ${ }^{4,5}$. These models have to be standardized and need to mimic the human clinical situation as close as possible. Previous studies have investigated closed induction ${ }^{6-8}$ of the fracture and open surgical procedures, ${ }^{5,9}$, with differences in osteotomy size (critical sized segmental defects vs non-critically sized), and different fixation techniques as bridging plates ${ }^{10-12}$, intramedullary nails ${ }^{7,13,14}$ and external fixators ${ }^{15,16}$.

Several factors have been shown to influence bony healing such as the biomechanical environment (interfragmentary instability), inadequate blood supply $^{17}$ as well as the defect size ${ }^{18,19}$. Availability of knockout mice and senescence altered mice allows a broad spectrum of molecular biological based investigations $^{20}$ into developmental biological issues such as bone and cartilage formation ${ }^{9,12,17}$ in combination with these different healing models.

Another key player in adequate fracture healing is the periosteum and integrity of the periosteum must be retained to achieve a successful fracture healing ${ }^{21}$. The periosteum consists of a thin, well vascularized and innervated layer along the cortex of the bone and is primarily composed of osteogenic and fibroblastic cells ${ }^{22}$. Especially during the soft callus formation, the periosteum has a major influence on fracture repair as the periosteal progenitor cells will differentiate into osteoblasts and, mainly, chondrocytes ${ }^{23,24}$. Consequently, in the present study, we hypothesize that periosteal cauterization would induce a significant and substantial delay in the bone healing process in mice. The aim of the current project is to describe and characterize the delayed healing process so that this developed novel model can be used for future biomechanical and molecular research to investigate the delayed bone healing process or its treatment.

\section{MATERIALS AND METHODS}

\section{Animals and study design}

A total of 87, 20-25 week old, skeletally mature, female, C57BL/6 mice (RCC Ltd, Füllingsdorf, Switzerland) were used in this study. Mice were housed socially in group cages with water and a standard maintenance diet (Provimi, Provimi 
Kliba AG, Kaiseraugst, Switzerland) ad libitum and with a 12-hour day-night cycle. Before the surgical procedures, mice were randomly assigned to the control group or the periosteal cauterization group and each group of mice was equally subdivided into five sub-groups for different follow-up times (7, 14, 21, 28 and 42 days, see table 4.1 for the numbers of mice per group, analysis type and time of follow-up).

The ethical committee of the Canton of Grison, Switzerland approved the experimental set-up and all (surgical) procedures conducted in this study.

TABLE 4.1 Randomization of mice per study group and conducted analysis

\begin{tabular}{llllll}
\hline \multicolumn{7}{c}{ Days of follow-up } & & \\
\hline Group & 7 days & 14 days & 21 days & 28 days & 42 days \\
\hline Control & 8 & 10 & 10 & 9 & 8 \\
\hline Periosteal cauterization & 8 & 9 & 8 & 8 & 9 \\
\hline analysis & $\mu \mathrm{CT}$ & $\mu \mathrm{CT}$ & 4 -point bending & 4 -point bending & 4 -point bending \\
& $\mathrm{X}-$ ray & $\mathrm{X}-$ ray & $\mu \mathrm{CT}$ & $\mu \mathrm{CT}$ & $\mu \mathrm{CT}$ \\
& & histology & X-ray & X-ray & X-ray \\
& & histology & histology & histology \\
\hline
\end{tabular}

\section{Anaesthesia, analgesia and surgical procedure}

General anaesthesia, analgesia and the surgical approach and postoperative pain treatment were carried out as previously described ${ }^{12,25}$. Briefly, the mice were operated under general anaesthesia using isoflurane after obtaining pre-emptive analgesia consisting of buprenorphine (Temgesic), which was continued for 24 hours every 8 hours postoperatively. Additionaly, mice received paracetamol per os for five days. In mice that were assigned to the periosteal cauterization group, a $0.8 \mathrm{~mm}$ thick titanium foil was pulled tight around the mid-shaft of the femur, held with forceps and an electrome was used to cauterize the periosteum circumferentially for 0.5 seconds with use of a protective Teflon cover around the other tissues. (see figure 4.1). In all animals, a 4-hole internal fixating plate (Titanium, 7.0 × $1.5 \times 0.7$ mm, MouseFix ${ }^{\text {TM }}$, RISystem AG, Davos, Switzerland) ${ }^{11}$ was placed on the lateral aspect of the femur and, after predilling with a $0.33 \mathrm{~mm}$ drill bit, secured with four $2.0 \mathrm{~mm}$ angular stable screws (MouseFix ${ }^{\mathrm{TM}}$, RISystem AG, Davos, Switzerland). Following fixation, a $0.45 \mathrm{~mm}$ mid-diaphyseal femoral gap osteotomy was performed by using a Gigli wire saw and irrigation with 0.9\% $\mathrm{NaCl}$. In the group with cautery, the osteotomy was placed in the middle of the periosteal injury resulting in a $0.25 \mathrm{~mm}$ wide strip of injured periosteum on the proximal and distal side of the gap. To induce secondary healing, the screws were loosened half a turn to some degree of instability into the fixation ${ }^{25}$. Free weight 
bearing was allowed immediately after recovery from anaesthesia.

Animals were euthanized using $\mathrm{CO}_{2}$ following the different time periods of fracture healing as shown in table 4.1 and both the right femur which underwent the osteotomy as the untouched left femur from each mouse were excised.

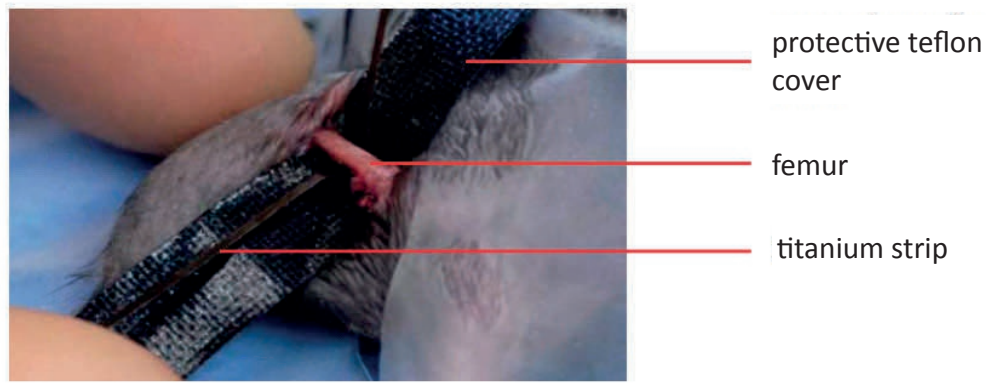

Figure 4.1. Cauterization of periosteum. During the surgical procedure, a titanium strip is placed circumferentially around the femur and subsequently attached to the cauterization device. A protective Teflon cover is placed under the femur and over surrounding soft tissues.

\section{Mechanical testing}

In mice euthanized after 21, 28 and 42 days of healing, the plate was gently removed and both femora were immediately tested in non-destructive 4-pointbending (ElectroForce 3220, Bose ESG, Eden Prairie, MN, USA). Femora were bent with the former plate position on the compression side at $2.1 \mathrm{deg} / \mathrm{min}$ to 4.5 Nmm. The linear portion of the curve was used to calculate the bending stiffness. Each femur was tested 3 times. The healing femur stiffness were averaged and normalized by the contralateral intact femur stiffness. Bones from earlier time points (1, 7 and 14 days) were too fragile to test due to insufficient bone healing.

\section{Micro-computed tomography analysis}

All bones were analysed by Micro-computed tomographic $(\mu \mathrm{CT})$ imaging $(\mu \mathrm{CT}$ 40, Scanco Medical, Bassersdorf, Switzerland): after excision and gentle removal of the plates (time points 7 and 14 days) or mechanical testing (time points 21, 28 and 42 days) all osteotomized femora were fixed in 100\% methanol. $\mu$ CT was performed as described in previous studies ${ }^{12,25}$ to evaluate the fracture gap of all bones. Three-dimensional reconstructions with a special resolution/voxel size of $12 \mu \mathrm{m}$ were made and based on a histogram of attenuation distribution, tissue was segmented into two types: woven bone (low mineralization, 14.5 to 36.0\% of maximal gray value) and lamellar bone (high mineralization, > 36\%). 
For precise quantitative analysis, different regions of interest (ROIs) were defined (see figure 4.2 for a schematic overview). The largest, total region of interest (TOT) included the entire scanned volume between the most proximal and distal placed screws. The periosteal region (PER) comprised any new bone tissue starting at the outer cortical boundary of the femora and extending radially outward, while the endosteal region (END) contained all newly formed bone within the medullary cavity, i.e. within the inner cortical boundary of both fragments. The actual fracture gap (GAP) was defined as the space between both fragments and its extension radially outward. The GAP region included only newly formed tissue, any bone fragments and original mid-diaphyseal cortex were excluded.
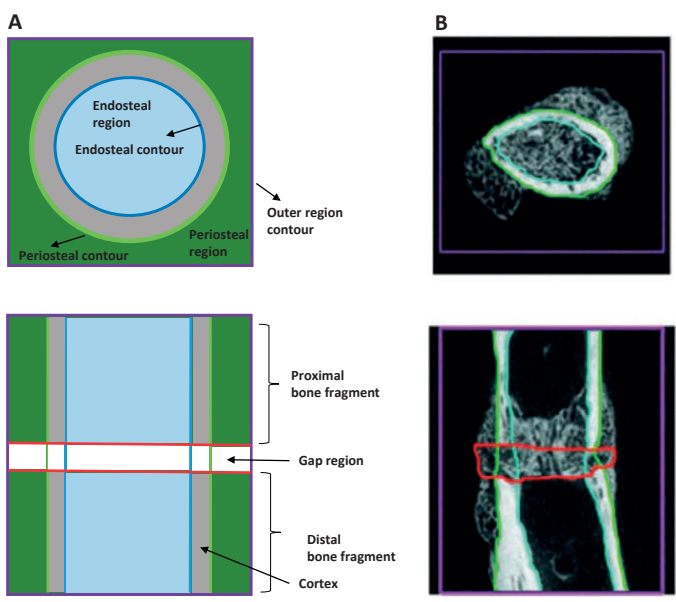

Figure 4.2. Definition of regions of interest during micro-CT analysis. Schematic representation of the four regions of interest: total region (TOT), periosteal region (PER), endosteal region (END) and the gap (GAP) between the fracture parts (panel A). The border of the complete investigated region (TOT) is the complete surrounding of the tissue between the most proximal and distal placed screws (purple borders). Green and light blue borders represent the periosteal and endosteal contours and regions of interest. The GAP region is marked by red lines at the osteotomy site. In panel $\mathrm{B}$, before mentioned borders are drawn in a representative micro-CT image. In both panel A and B, the upper image shows a transverse cross-sectional representation of the femur and the lower image a longitudinal representation.

\section{Radiographic score}

Blinded postoperative and post-mortem radiographs as well as cross-sectional CT images per sample of all time points were graded based on callus formation, rebridging of the cortices and callus remodelling using the radiographic scoring scale of Garrett et $a \mathbb{R}^{6}$ (see table 4.2). Radiographs provided the overview of fragment alignment and callus formation while 16 two-dimensional CT-cross- 
sections, equally spaced in the central part of the GAP region were assessed blindly by three medically trained investigators. Results are presented as median with maximum score.

TABLE 4.2 Radiographic-scoring scale according to Garrett et al ${ }^{6}$. Based on rebridgement of the cortices and acceleration of healing.

\begin{tabular}{ll}
\hline Score & Definition \\
\hline 0 & No bridging, no callus formation \\
\hline 1 & No bridging, initiation of a small amount callus \\
\hline 3 & No bridging, obvious callus formation near fracture \\
\hline 5 & $\begin{array}{l}\text { Ro bridging, marked callus formation near and around fracture site } \\
\text { fracture site }\end{array}$ \\
\hline 6 & $\begin{array}{l}\text { Rebridging of at least one of the cortices, marked and complete callus formation around } \\
\text { fracture site }\end{array}$ \\
\hline 7 & Rebridging of both cortices, and/or some resolution of the callus \\
\hline
\end{tabular}

\section{Histology}

For histological analysis, femora of both groups after 14, 21, 28 and 42 days of fracture healing were decalcified (12.5\% EDTA with $1.25 \% \mathrm{NaOH}$ ), embedded in paraffin and cut into $6 \mu \mathrm{m}$ thick sections. Immunohistochemistry was performed for collagen II and collagen X to compare the time course for chondrocyte maturation and differentiation as described previously ${ }^{25}$. Sections were counterstained with haematoxylin and eosin to provide a clear overview of the images. Evaluation was performed qualitatively (Axioplan. Carl Zeiss AG, Feldbach Switzerland) using transmitted light at 50x magnification.

\section{Statistical analysis}

Normal distribution of all subgroups was tested using Shapiro-Wilks test. An analysis of variance was performed with periosteal injury and healing time as factors in a full factorial general linear model using post hoc Tukey correction. Differences at specific time points were tested with One-way ANOVA for "time" and independent T-test for "treatment" using post hoc Bonferroni correction (significance threshold $\mathrm{p}<0.01$ ). Inter-observer agreement was tested using Fleiss' Kappa and afterwards, differences in radiographic scoring between groups were analysed by nonparametric Kruskal-Wallis test and Mann Whitney U test. P-values below 0.05 were considered statistical significant unless stated otherwise. Analysis was performed using GraphPad Prism 6 (GraphPad, San Diego, California, USA). Data in this paper are represented as means and standard error of the mean (SEM). 


\section{RESULTS}

\section{Mechanical testing}

Bending stiffness assessed during 4-point mechanical bending tests of healing bones at 21, 28 and 42 days post-surgery was significantly lower for the periosteal injury group when compared to control animals (all $\mathrm{p}<0.05$, figure 4.3). In both groups stiffness was significantly higher at 42 days of healing when compared to both previous time points (both $\mathrm{p}<0.001$ ).

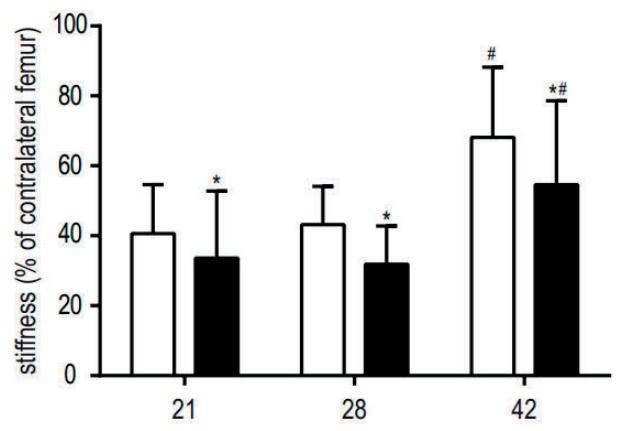

Figure 4.3. Biomechanical testing. Results of 4 point bending stiffness after 21, 28 and 42 days of healing, results are presented as \% stiffness compared with the contralateral (unfractured) femur. Control animals are shown in white bars, the periosteal cauterization group in black.

Significance: *: p $<0.05$ when compared to control. \#: p < 0.001 when compared to day 21 or 28.

\section{Micro-computed tomography analysis}

Reconstructed 3-dimensional $\mu \mathrm{CT}$ images (figure 4.4) demonstrated that in the control group, fracture healing took place with immediate initial callus formation and subsequent resorption (day 28, fig. 4.4E) and remodelling of the callus (day 42, fig. 4.4G). In contrast, in the periosteal injury group no periosteal reactions were noticeable at the beginning (fig. 4.4B, D and F) and callus formation was both delayed and reduced. By day 42 (fig. $4.4 \mathrm{H}$ ), the fracture healing process had started and an immature callus was observed in the periosteal cauterization group. 


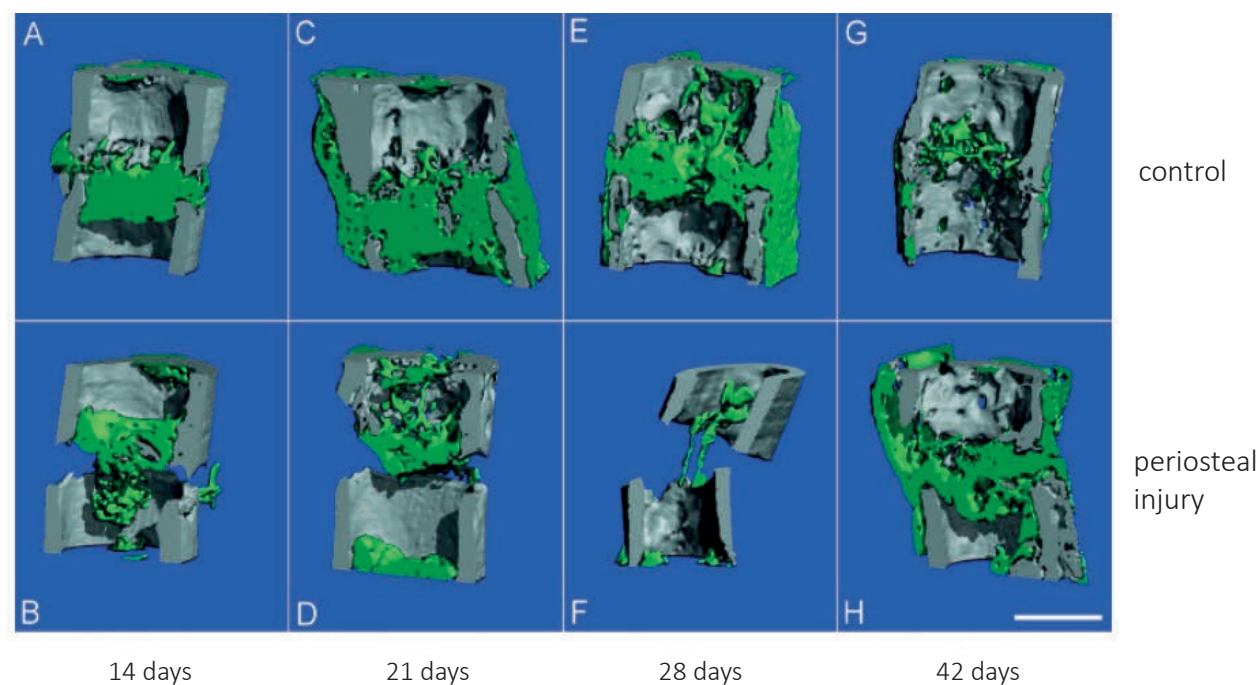

Figure 4.4. Reconstructed 3-dimensional qualitative micro-CT images. Representative micro-CT images of femurs after 14 (panel A and B), 21 (C and D), 28 (E and F) and 42 ( G and H) days of healing in the control group and periosteal injury group respectively. Highly mineralized tissue is shown in gray, a lower degree of mineralization in green. Scale bar in panel H represents $1 \mathrm{~mm}$ and can be translated to the other panels.

Quantitative $\mu \mathrm{CT}$ evaluation of the TOT region of interest showed that reduced volumes of woven bone are formed during the healing process in the periosteal injury group before day 42 ( $\mathrm{p}<0.05$; Figure 4.5A). In both groups, woven bone volumes changed over time. Mice in the control group showed a substantially steeper increase in woven bone volumes, especially between 14 and 21 days in comparison with animals that underwent periosteal cauterization. After 21 days, bone volumes in control animals reached a maximum, whereas woven bone volumes in the periosteal injury group increased at a lesser, steady rate until day 28 ( $\mathrm{p}<0.05$ when compared with measured femora after 7 and 14 days of fracture healing). Thereafter, the volume of woven bone decreased in the control group until the end of the experiment after 42 days when compared with samples collected after 21 and 28 days of fracture healing (both $p<0.0001$ ). In the periosteal injury group, the woven bone volumes remained elevated at 42 days of healing.

Woven bone volumes in the PER (Fig. 4.5B) and END (Fig. 4.5C) regions showed again a peak at 21 days of fracture healing (both $p<0.0001$ when compared to 7 days), which afterwards subsequently decreased until the end of the experimental period at 42 days (both $\mathrm{p}<0.0001$ when compared with 21 days of healing). In the periosteal injury group, an increase was observed in woven bone volumes in the PER region between 7 and 21 days of healing, which afterwards stayed almost 
constant at a plateau level until the end of the experimental period (all $\mathrm{p}<0.05$ when compared to samples collected after 7 days of healing). A similar pattern of bone volumes was present in the END region during fracture healing in mice with periosteal injury. The peak in periosteal woven bone volume (Fig. 4.5B) in control animals was a 2-fold higher when compared with the periosteal injury group ( $\mathrm{p}<0.001)$ and to a lesser extend also in the endosteal region of interest $(\mathrm{p}<0.05)$.

Periosteal injury suppressed callus growth in the GAP region with significantly less woven bone volumes between 7 and 28 days of fracture healing $(\mathrm{p}<0.01$ at every time point) and a maximum volume which is a 2- to 3-fold lower when compared with normal fracture healing in control mice (Fig. 4.5D).

Total lamellar bone volumes did not differ significantly between 7 and 42 days in control animals as well as mice with periosteal injury (Fig. 4.5E). Volumes of lamellar bone in the PER region of interest (Fig. 4.5F) increased significantly in both groups until the end of the experiment (both $\mathrm{p}<0.0001$ ), however, at the end of the experimental period, femora in the control group showed a 2 -fold higher bone volumes compared with samples after periosteal injury ( $<<0.05)$. Lamellar bone volumes in the endosteal region (Fig. 4.5G) showed an increase in until postoperative day 28 in control mice $(\mathrm{p}<0.05)$ and subsequent diminished volumes at 42 days ( $\mathrm{p}<0.001$ ). In the periosteal injury group, the peak of lamellar bone volume was at the end of the experimental period at an increase of $~ 50 \%$ when compared with measurements taken after 7 days $(\mathrm{p}<0.01)$. Finally, in the GAP region between the proximal and distal part of the femur, lamellar bone volumes increased by 50 -fold in the control group ( $\mathrm{p}<0.001$ when compared to day 7 ) and about 25-fold in mice with compromised healing ( $p<0.01, \mathrm{p}<0.05$ between both groups of animals).

\section{Radiographic score}

Kappa values for each of the three observer pairs were $0.49,0.38$ and 0.34 respectively. The overall inter-observer agreement was fair (0.40). In the control femora, the osteotomies healed progressively with lower variation among animals over time (Fig. 4.6). In the group with periosteal injury, a higher variability in healing progress was registered, especially after 28 days of healing. However, after 42 days, the radiographs demonstrated a step forward in healing with consistently higher scoring in these animals. Radiographic grading indicated consistent earlier and more advanced healing in control animals when compared to mice with periosteal injury starting at post-operative day $14(\mathrm{p}<0.05)$ and until the end of the experimental period at day 42 where cortical bridging was not always attained in the periosteal injury group. The delay in score magnitude ranged between approximately 1-2 weeks. 
A

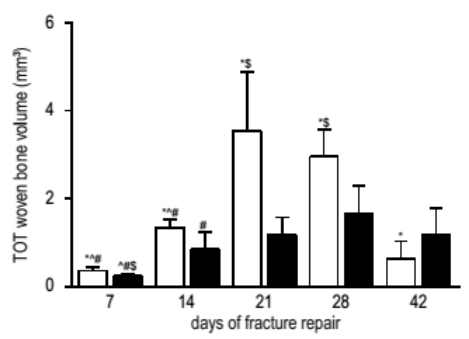

C

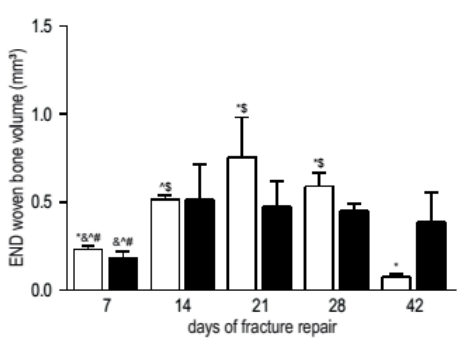

E

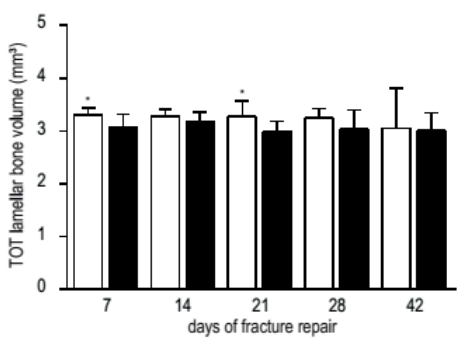

G

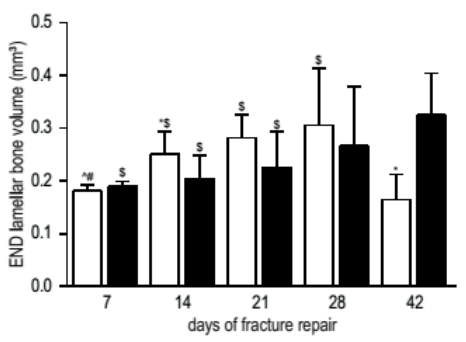

B

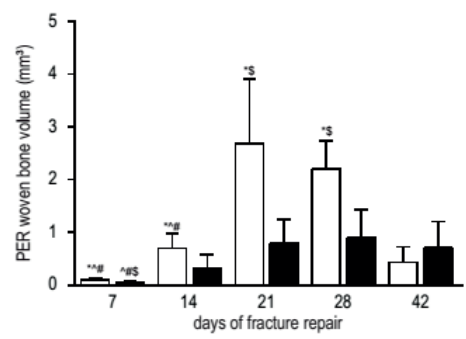

D

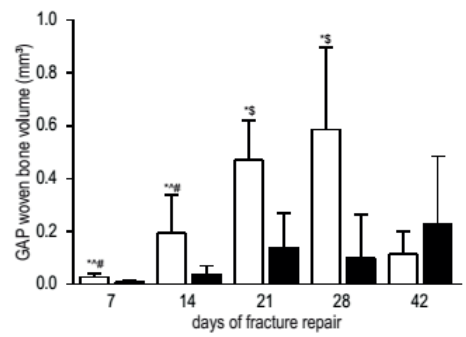

F

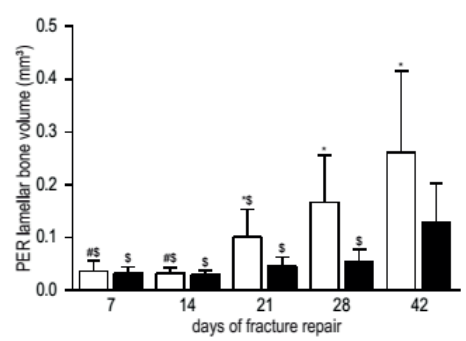

H

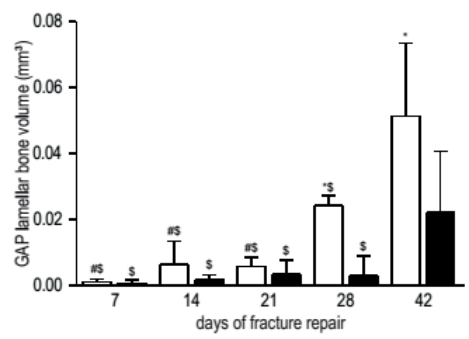

$\square$ control

periosteal injury

Figure 4.5. Woven and lamellar bone volumes in all 4 regions of interest. White bars represent control animals without periosteal cauterization, black bars mice with periosteal injury. TOT: total region, PER: periosteal region, END: endosteal region and GAP: osteotomy gap between proximal and distal part of the femur. Panel A, B, C and D show volumes of woven bone in the four different regions of interest, and panel E, F, G and H volumes of lamellar bone. *: p < 0.05 when compared to periosteal injured mice at same time point. \&: p < 0.05 when compared with 14 days of healing. $\wedge$ : p < 0.05 when compared with 21 days of healing. \#: p $<0.05$ when compared with 28 days of healing. \$: p $<0.05$ when compared with 42 days of healing. 


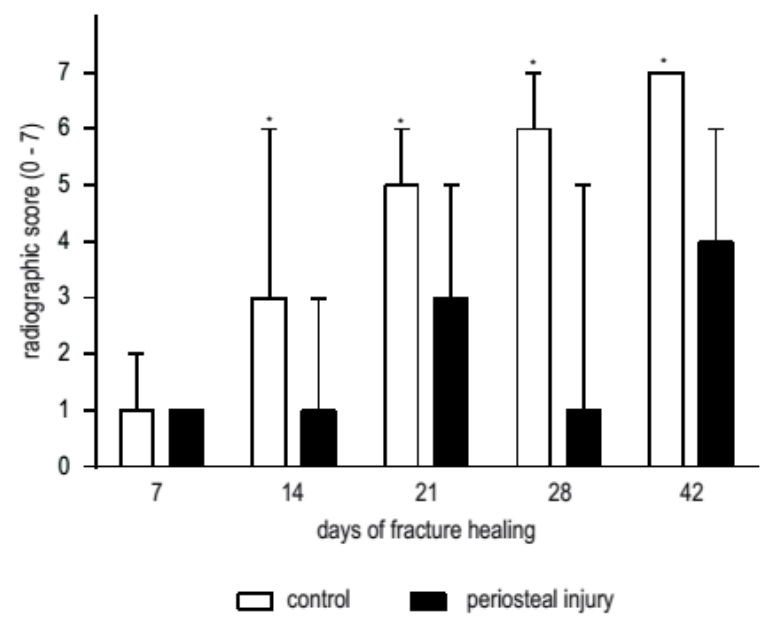

Figure 4.6. X-ray evaluation of fracture healing. Results of radiographic evaluation of healing at day 7, 14, 21, 28 and 42 after the femur osteotomy. Evaluation was performed by three independent researchers according to the scoring-scale by Garrett et al, with results presented as median with maximum score. White bars represent control mice, black bars show mice with periosteal cauterization. $*: \mathrm{p}<0.05$.

\section{Histology}

Histological results corroborated the quantitative outcome from the $\mu \mathrm{CT}$ analysis. Based on the expression of collagen II (Col II) and collagen X ( Col X), on the formation and resorption of cartilaginous tissue in the gap and on the bridging between the fragments, the healing course was clearly prolonged in the group with periosteal injury. In femora of the control group, a more robust expression of Col II and Col X (respectively in figures 4.7 and 4.8) was observed between postoperative days 14 and 28. After 14 days, the callus in the fracture gap consisted of woven bone in combination with cartilage which was mainly located in the centre of the gap region (Fig. 4.7A and 4.8A). At day 21 a more massive periosteal reaction was visible, whose cartilaginous portion was increasingly replaced by woven bone as evidenced by intense Col X staining (Fig. 4.7C and 4.8C). At day 28 (Fig. 4.7E and 4.8E) both cortices were bridged with woven bone and remodelling had already started, noticeable by the advanced stage of callus resorption around the periosteum and in the endosteal cavity. In the group with periosteal injury, woven bone formation and the amount of cartilage was delayed, as demonstrated by the expression of $\mathrm{Col}$ II and Col X. After 14 days (Fig. 4.7B and 4.8B) only connective tissue and no callus was visible in the fracture gap. At day 21 (Fig 4.7D and 4.8D) Col II and Col $\mathrm{X}$ were detected representing the amount of cartilage located within the cortical boundaries of the two fragments. On day 28 after the surgical procedure (Fig. $4.7 \mathrm{~F}$ and $4.8 \mathrm{~F}$ ), an extensive cartilaginous 
callus was formed and included some mild amounts of woven bone; reflecting a delayed reaction to a persistent instability of the fracture fixation and mechanically inadequate stabilization with fibrous tissue and cartilage.

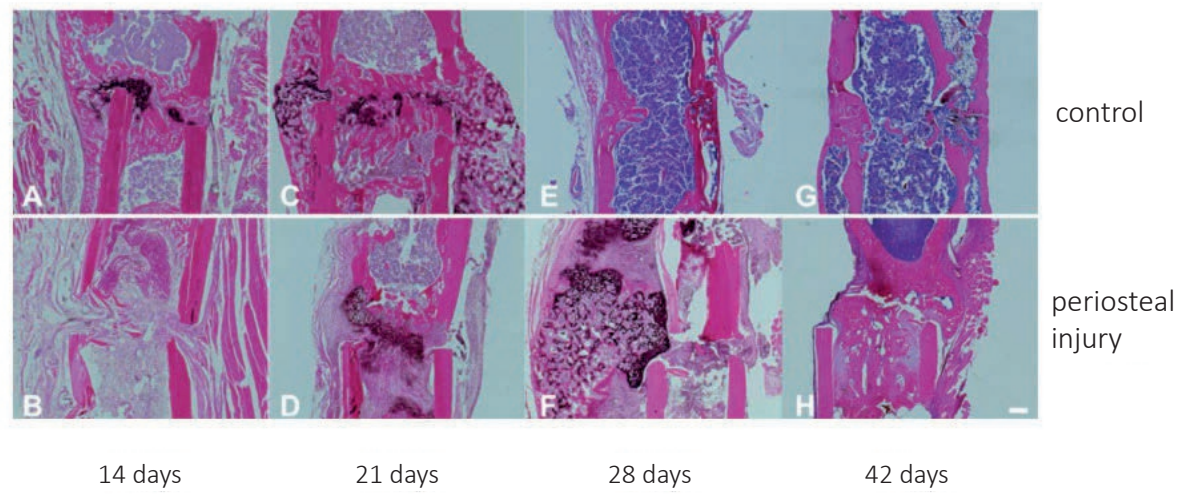

Figure 4.7. Collagen II immunohistochemistry. Panels A, C, E, and G represent mid-sagittal femur histology slides stained for collagen II after 14, 21, 28 and 42 days respectively, with H\&E counter staining. In panels $\mathrm{B}, \mathrm{D}, \mathrm{F}$ and $\mathrm{H}$ femurs of mice with periosteal injury are represented at the same time points. Images are made at a 50x magnification, the scale bar represents a size of $200 \mu \mathrm{m}$.

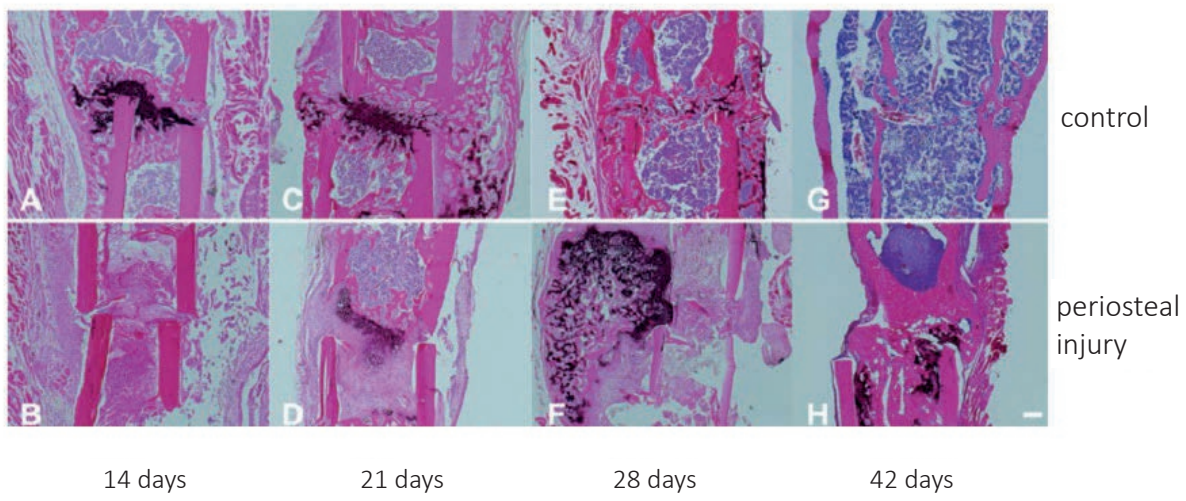

Figure 4.8. Collagen $\mathrm{X}$ immunohistochemistry. Panels A, C, E, and G represent mid-sagittal femur histology slides stained for collagen $\mathrm{X}$ after 14, 21, 28 and 42 days respectively, with H\&E counter staining. In panels $\mathrm{B}, \mathrm{D}, \mathrm{F}$ and $\mathrm{H}$ femurs of mice with periosteal injury are represented at the same time points. Images are made at a 50x magnification, the scale bar represents a size of $200 \mu \mathrm{m}$. 


\section{DISCUSSION}

The aim of the current project was to develop an in vivo murine model for delayed union development, as an intermediary between normal fracture healing and the development of nonunions, for future possibilities in biochemical and molecular research to investigate enhanced and deficient bone healing processes. Results demonstrated that the fracture gap obtained after a standardized osteotomy reduced with semi-rigid internal plate-screw osteosynthesis and combined with periosteal injury prolonged the healing period for 7 to 14 days, with callus formation volumes after 42 days of fracture healing which were comparable with callus after 21-28 days in the control group. In contrast, in the control group without periosteal cauterization resorption of the fracture callus via remodelling processes was well advanced with restoration of the femur diameter and reconstruction of the medullary canal. Therefore, this model of delayed fracture healing provides an ideal intermediate between normal fracture healing and nonunion development, whereas larger sized osteotomies would result in critical segmental defects resulting in nonunion development without the ability to assess the enhanced healing capabilities of future bone healing strategies.

Quantitative results from the $\mu \mathrm{CT}$ analysis showed that as a consequence of the periosteal injury, the typical healing response was inhibited with the amount of woven bone in and mostly around the osteotomy was significantly reduced. Bridging of the proximal and distal fragments with mineralized lamellar bone was delayed accordingly. Radiographic analysis showed similar patterns in fracture repair with a 1 to 2 week delay in the periosteal injury group. Immunohistochemical evaluation on formation, maturation and hypertrophy of chondrocytes using Col II and Col X markers also demonstrated a shift in the fracture repair response as shown in figure 7 and 8 . In the periosteal injury group, the normal healing cascade was delayed and prolonged with fibrous connective tissue and cartilage still present in the gap region, chondrocytes which just started to hypertrophy, limited presence of woven bone and no complete bridging of the cortices evident. As a result, postponed healing delayed functionality as bending stiffness increased over time for both the control group as the mice with periosteal injury. Stiffness at the end of the experimental period was significantly higher in control animals when compared with periosteal injured mice due to a larger callus supporting the osteotomy and a higher degree of bone mineralization. As other isoforms, i.e., collagen I are mainly found in mature bone, these were not investigated in the present study. Collagen III, which is found in scar tissue and connective tissue, next to the blood vessel walls, has been reported to regulate osteoblastogenesis ${ }^{27,28}$. However, the most pronounced delayed union and nonunions in our model are observed after day 28 whereas collagen III is mainly found between the fifth and 
twentieth postoperative day and additionally is does not significanlty affect the callus volume in the early stages of fracture repair ${ }^{27}$, therefor making collagen III a less reliable marker in our current investigation.

The electro cauterization procedure performed in this study destroyed the integrity of the periosteum on the proximal and distal side of the osteotomy gap. Disruption of the periosteum leads to a markedly impaired blood supply $22,29-31$ and subsequent to a reduced release and proliferation of various cell types and to a reduced capacity to form bone and cartilage ${ }^{17,32}$. The critical role for the periosteum explains the obtained results in this study that in the periosteal injured group of mice during the first two weeks of fracture healing neither chondrocytes nor osteoblast-specific cells were migrating to the osteotomy gap and only fibrous tissue did develop.

Extensive reviews have been published on in vivo models of fracture healing and delayed union and nonunion development in rodents ${ }^{33-35}$. A wide range of different models have been created to study biomechanical and biomolecular processes during fracture repair and compromised fracture healing.

Standardized closed fracture models have been developed which induce fractures by three of four-point bending ${ }^{8}$ or using a blunt guillotine combined with a dropping weight ${ }^{6}$. In these models, the fracture will represent a more realistic situation as is seen clinically with a better containment of the fracture hematoma. As compared to our newly developed model, a disadvantage is, as this is not a model of compromised fracture healing, that a relatively low number of delayed unions/nonunions which will occur decreasing the usability for studying the biomolecular and biomechanical processes during delayed fracture repair. Also, since there is relatively thin soft tissue coverage of the tibia, its influence on fracture healing and possible interplays between different tissues is difficult to assess in this model ${ }^{34}$.

A range of different intramedullary fixation methods are presented in literature used in closed fracture models ${ }^{7}$ and in open ${ }^{5,9}$ surgical procedures. Minimally invasive methods used are accompanied by a lack of rotational and axial stability and as a result have a high risk of dislocation ${ }^{7}$, making them not useful in standardized delayed union research. More adequate models using intramedullary pins are accompanied by locking nails ${ }^{8,13}$ or compression screws ${ }^{14}$ making it possible to use segmental defects for studying compromised fracture healing. However, all intramedullary fixation techniques severely damage the medullary canal, making it impossible to study the different endosteal processes during healing of the bone ${ }^{34}$.

Until now, delayed union studies in mice and rats have been conducted using external fixators ${ }^{15,16}$, intramedullary pins $^{5,9,36}$ or no fixation at all ${ }^{17}$. The use of unilateral or circular external fixation devices ensures minimal disturbances of 
the fracture/osteotomy location during healing but also in subsequent analysis. However, the relatively high weight of the fixators and possible excessive micromovement when using unilateral fixators will increase the unpredictability of the obtained results 34,35 .

Plate-screw osteosynthesis with locking plates and screws ${ }^{11}$ as used in the current study is designed for minimal periosteal contact and can as such be used to investigate influence of periosteal modification on fracture healing and keeping the advantages of an intact medullary canal when compared with the intramedullary fixation methods. Reproducible results have been obtained in the current study and previously ${ }^{10,12}$.

Although mice are not an exact model for human fracture healing, since rodents lack a Haversian system but use comparable resorption cavities for bone remodeling ${ }^{4,37}$, a major advantage of murine models is the reduced time (and costs) necessary for experiments since the healing process under normal circumstances takes around 3 weeks until there is no detectable motion between the fracture parts $^{33,38}$. In the current investigation, we had better controlled biomedical conditions as compared to other fixation techniques ${ }^{39-41}$, and advantages over models which use tibial fracture healing as the straight longitudinal axis of the femur makes standardized fracture stabilization and accuracy of biomechanical testing easier and more reproducible. Recently, titanium covered PEEK (polyether ether ketone) are developed and used which mimic the titanium surface of human osteosynthesis materials ${ }^{10}$. From an ethical point of view, every animal can then be monitored multiple times and during longer periods and without the need of euthanasia prior to collecting data, which is in compliance with the principles of reduction, replacement and refinement in lab animal experiments.

Mice also have a broad range of possibilities for usage of gene-targeted (knock-out/ knock-in) animals, which enables molecular mechanistic studies on bone repair ${ }^{42}$ and different existing models are present e.g. in research aimed at osteoporosis based fracture healing in senescence accelerated mice ${ }^{43}$. The periosteal injury model discussed in this current study has been used in the recently published study on the influence of nitric oxide (NO) deficiency on delayed bone healing and nonunion development ${ }^{12}$. In short, in this study, knockout mice deficient for nitric oxide synthase (a key enzyme necessary for NO production) showed nonunion development when compared with normal wild type control animals, after a femur osteotomy combined with periosteal cauterization, as used in the current study. At the end of the experimental period after 42 days of fracture healing, the deficient animals showed no presence of callus formation and bone volumes which were between 2- and 5-fold lower when compared with mice in the control situation. 
When interpreting the obtained results, some limitations need to be considered. In the periosteal injury group, some longer time points for the follow up period would be needed to assess if the healing process continues and subsequently results in remodelling of the callus as is shown in the control group. With these extra time points, the final delay in healing could be assessed. Next to this, we only investigate one factor leading to the delay in fracture healing and control other confounding factors such as the biomechanical environment. In this model for delayed fracture healing this is a strength resulting in reproducible data, however since bone healing in general is a multifactorial process, further research is needed into other influential factors. A final minor point of attention is the fair interobserver agreement which was reached in the radiographic analysis, however, this limited value underscores the micro-computed tomography results which show comparable and significant quantified results of bone and callus formation.

In conclusion, a moderate fracture gap produced by osteotomy and fixated by flexible plate-screw osteosynthesis in combination with additional periosteal injury induced by electro cauterization leads to a delayed union development in a murine in vivo model. The periosteal injury induced a delay of healing time of 1-2 weeks compared to control samples, visible as callus formation and gap bridging and the presence of collagen expression within the gap region. The observed delay is considered to be clinically relevant since normalized by averaged healing time in mice ( 4 weeks) ${ }^{41}$ and humans (16 to 20 weeks), it can be extrapolated that a delay of about 1-2 weeks in mice would correspond to delayed healing in humans by around 4-6 weeks. In the future, this mouse model with periosteal injury can be used to evaluate basic research questions regarding involvement of certain pathways or genes or to develop diagnostic tools and treatment options, in a model that provides a continuum between normal fracture healing and the development of nonunions. 


\section{REFERENCES}

1. Einhorn TA. Enhancement of fracture-healing. J Bone Joint Surg Am 1995; 77(6): 940-56.

2. Mills LA, Simpson AH. The relative incidence of fracture non-union in the Scottish population (5.17 million): a 5-year epidemiological study. BMJ Open 2013; 3(2).

3. Hayda RA, Brighton CT, Esterhai JL, Jr. Pathophysiology of delayed healing. Clinical orthopaedics and related research 1998; (355 Suppl): S31-40.

4. Holstein JH, Garcia P, Histing T, et al. Advances in the establishment of defined mouse models for the study of fracture healing and bone regeneration. J Orthop Trauma 2009; 23(5 Suppl): S31-8.

5. Garcia P, Holstein JH, Maier S, et al. Development of a reliable non-union model in mice. J Surg Res 2008; 147(1): 84-91.

6. Bonnarens F, Einhorn TA. Production of a standard closed fracture in laboratory animal bone. Journal of orthopaedic research : official publication of the Orthopaedic Research Society 1984; 2(1): 97-101.

7. Histing T, Holstein JH, Garcia P, et al. Ex vivo analysis of rotational stiffness of different osteosynthesis techniques in mouse femur fracture. Journal of orthopaedic research : official publication of the Orthopaedic Research Society 2009; 27(9): 1152-6.

8. Holstein JH, Menger MD, Culemann U, Meier C, Pohlemann T. Development of a locking femur nail for mice. Journal of biomechanics 2007; 40(1): 215-9.

9. Garcia P, Holstein JH, Histing T, et al. A new technique for internal fixation of femoral fractures in mice: impact of stability on fracture healing. Journal of biomechanics 2008; 41(8): 1689-96.

10. Inzana JA, Schwarz EM, Kates SL, Awad HA. A novel murine model of established Staphylococcal bone infection in the presence of a fracture fixation plate to study therapies utilizing antibioticladen spacers after revision surgery. Bone 2015; 72: 128-36.

11. Matthys R, Perren SM. Internal fixator for use in the mouse. Injury 2009; 40 Suppl 4: S103-9.

12. Meesters DM, Neubert S, Wijnands KA, et al. Deficiency of inducible and endothelial nitric oxide synthase results in diminished bone formation and delayed union and nonunion development. Bone 2016; 83: 111-8.

13. Garcia P, Herwerth S, Matthys R, et al. The LockingMouseNail--a new implant for standardized stable osteosynthesis in mice. J Surg Res 2011; 169(2): 220-6.

14. Holstein JH, Matthys R, Histing T, et al. Development of a stable closed femoral fracture model in mice. The Journal of surgical research 2009; 153(1): 71-5.

15. Dickson GR, Geddis C, Fazzalari N, Marsh D, Parkinson I. Microcomputed tomography imaging in a rat model of delayed union/non-union fracture. J Orthop Res 2008; 26(5): 729-36.

16. Rontgen V, Blakytny R, Matthys R, et al. Fracture healing in mice under controlled rigid and flexible conditions using an adjustable external fixator. Journal of orthopaedic research : official publication of the Orthopaedic Research Society 2010; 28(11): 1456-62.

17. Lu C, Miclau T, Hu D, Marcucio RS. Ischemia leads to delayed union during fracture healing: a mouse model. Journal of orthopaedic research : official publication of the Orthopaedic Research Society 2007; 25(1): 51-61. 
18. Schmidmaier G, Capanna R, Wildemann B, Beque T, Lowenberg D. Bone morphogenetic proteins in critical-size bone defects: what are the options? Injury 2009; 40 Suppl 3: S39-43.

19. Claes LE, Heigele CA, Neidlinger-Wilke C, et al. Effects of mechanical factors on the fracture healing process. Clin Orthop Relat Res 1998; (355 Suppl): S132-47.

20. Einhorn TA. The cell and molecular biology of fracture healing. Clinical orthopaedics and related research 1998; (355 Suppl): S7-21.

21. Roberts SJ, van Gastel N, Carmeliet G, Luyten FP. Uncovering the periosteum for skeletal regeneration: The stem cell that lies beneath. Bone 2015; 70C: 10-8.

22. Allen MR, Hock JM, Burr DB. Periosteum: biology, regulation, and response to osteoporosis therapies. Bone 2004; 35(5): 1003-12.

23. Colnot C. Skeletal cell fate decisions within periosteum and bone marrow during bone regeneration. Journal of bone and mineral research : the official journal of the American Society for Bone and Mineral Research 2009; 24(2): 274-82.

24. Schindeler A, McDonald MM, Bokko P, Little DG. Bone remodeling during fracture repair: The cellular picture. Semin Cell Dev Biol 2008; 19(5): 459-66.

25. Grongroft I, Heil P, Matthys R, et al. Fixation compliance in a mouse osteotomy model induces two different processes of bone healing but does not lead to delayed union. J Biomech 2009; 42(13): 2089-96.

26. Garrett IR, Gutierrez GE, Rossini G, et al. Locally delivered lovastatin nanoparticles enhance fracture healing in rats. Journal of orthopaedic research : official publication of the Orthopaedic Research Society 2007; 25(10): 1351-7.

27. Miedel EL, Brisson BK, Hamilton T, et al. Type III collagen modulates fracture callus bone formation and early remodeling. J Orthop Res 2015; 33(5): 675-84.

28. Volk SW, Shah SR, Cohen AJ, et al. Type III collagen regulates osteoblastogenesis and the quantity of trabecular bone. Calcif Tissue Int 2014; 94(6): 621-31.

29. Dickson KF, Katzman S, Paiement G. The importance of the blood supply in the healing of tibial fractures. Contemporary orthopaedics 1995; 30(6): 489-93.

30. Oetgen ME, Merrell GA, Troiano NW, Horowitz MC, Kacena MA. Development of a femoral nonunion model in the mouse. Injury 2008; 39(10): 1119-26.

31. Raggatt LJ, Wullschleger ME, Alexander KA, et al. Fracture Healing via Periosteal Callus Formation Requires Macrophages for Both Initiation and Progression of Early Endochondral Ossification. The American journal of patbology 2014; 184(12): 3192-204.

32. Claes L, Eckert-Hubner K, Augat P. The effect of mechanical stability on local vascularization and tissue differentiation in callus healing. Journal of orthopaedic research: official publication of the Orthopaedic Research Society 2002; 20(5): 1099-105.

33. Garcia P, Histing T, Holstein JH, et al. Rodent animal models of delayed bone healing and nonunion formation: a comprehensive review. European cells \& materials 2013; 26: 1-12; discussion -4 .

34. Histing T, Garcia P, Holstein JH, et al. Small animal bone healing models: standards, tips, and pitfalls results of a consensus meeting. Bone 2011; 49(4): 591-9. 
35. Mills LA, Simpson AH. In vivo models of bone repair. J Bone Joint Surg Br 2012; 94(7): 865-74.

36. Aro H, Eerola E, Aho AJ. Development of nonunions in the rat fibula after removal of periosteal neural mechanoreceptors. Clinical orthopaedics and related research 1985; (199): 292-9.

37. Nunamaker DM. Experimental models of fracture repair. Clinical orthopaedics and related research 1998; (355 Suppl): S56-65.

38. Kilborn SH, Trudel G, Uhthoff H. Review of growth plate closure compared with age at sexual maturity and lifespan in laboratory animals. Contemp Top Lab Anim Sci 2002; 41(5): 21-6.

39. Le AX, Miclau T, Hu D, Helms JA. Molecular aspects of healing in stabilized and non-stabilized fractures. Journal of orthopaedic research : official publication of the Orthopaedic Research Society $2001 ; \mathbf{1 9}(1)$ : 78-84.

40. Thompson Z, Miclau T, Hu D, Helms JA. A model for intramembranous ossification during fracture healing. Journal of orthopaedic research : official publication of the Orthopaedic Research Society 2002; 20(5): 1091-8.

41. Hiltunen A, Vuorio E, Aro HT. A standardized experimental fracture in the mouse tibia. Journal of orthopaedic research : official publication of the Orthopaedic Research Society 1993; 11(2): 305-12.

42. Jacenko O, Olsen BR. Transgenic mouse models in studies of skeletal disorders. J Rheumatol Suppl 1995; 43: 39-41.

43. Egermann M, Heil P, Tami A, et al. Influence of defective bone marrow osteogenesis on fracture repair in an experimental model of senile osteoporosis. Journal of orthopaedic research : official publication of the Orthopaedic Research Society 2010; 28(6): 798-804. 



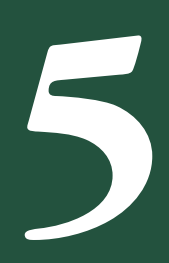

\title{
Deficiency of inducible and endothelial nitric oxide synthase results in diminished bone formation and delayed union and nonunion development
}

\author{
D.M. Meesters \\ S. Neubert \\ K.A.P. Wijnands \\ F.L. Heyer \\ S. Zeiter \\ K. Ito \\ P.R.G. Brink \\ M. Poeze
}

Bone 


\section{ABSTRACT}

\section{Background}

Between 5\% and 10\% of all fractures fail to heal adequately resulting in nonunion of the fracture fragments. This can significantly decrease a patient's quality of life and create associated psychosocial and socio-economic problems. Nitric oxide (NO) and nitric oxide synthases (NOS) have been found to be involved in fracture healing, but until now it is not known if disturbances in these mechanisms play a role in nonunion and delayed union development. In this study, we explored the role of endothelial and inducible NOS deficiency in a delayed union model in mice.

\section{Methods}

A $0.45 \mathrm{~mm}$ femur osteotomy with periosteal cauterization followed by platescrew osteosynthesis was performed in the left leg of 20-24 week old wild type, $\mathrm{Nos}^{2 /-}$ and $\mathrm{Nos}^{-/-}$mice. Contralateral unfractured legs were used as a control. Callus volume was measured using micro-computed tomography ( $\mu \mathrm{CT}$ ) after 28 and 42 days of fracture healing. Immuno histochemical myeloperoxidase (MPO) staining was performed on paraffin embedded sections to assess neutrophil influx in callus tissue and surrounding proximal and distal marrow cavities of the femur. After 7 and 28 days of fracture healing, femurs were collected for amino acid and RNA analysis to study arginine-NO metabolism.

\section{Results}

With $\mu \mathrm{CT}$, delayed union was observed in wild type animals, whereas in both $\mathrm{Nos}^{2 /-}$ and $\mathrm{Nos}^{-/-}$mice nonunion development was evident. Both knock-out strains also showed a significantly increased influx of MPO when compared with wild type mice. Concentrations of amino acids and expression of enzymes related to the arginine-NO metabolism were aberrant in NOS deficient mice when compared to contralateral control femurs and wild type samples.

\section{Conclusion}

In the present study we show for the first time that the absence of nitric oxide synthases results in a disturbed arginine-NO metabolism and inadequate fracture healing with the transition of delayed union into a nonunion in mice after a femur osteotomy. Based on these data we suggest that the arginine-NO metabolism may play a role in the prevention of delayed unions and nonunions. 


\section{INTRODUCTION}

Normal fracture healing is a process of partially overlapping phases of inflammation, callus formation and bone remodeling in which there is an interplay between various cells, growth factors and extracellular matrix ${ }^{1}$. However, five to ten percent of all patients experience difficulties during the healing process ${ }^{2}$ resulting in delayed union or nonunion of the fracture, indicated by persisting fracture lines and presence of a hypertrophic or atrophic callus ${ }^{3}$. Malnutrition, drug therapy, inadequate stabilization of the fracture and/or inadequate blood supply (i.e. periosteal injury) contribute to nonunion development ${ }^{4,5}$.

Adequate production of $\mathrm{NO}$ (nitric oxide), a free radical produced during the conversion of arginine into citrulline by nitric oxide synthases (NOSs) stimulates bone cells to regulate bone remodeling and influences vascular reactivity ${ }^{6-8}$. Furthermore, $\mathrm{NO}$ is suggested to stimulate polyamine production through the formation of ornithine, as precursors of collagen synthesis, ${ }^{9,10}$. An intricate interplay exists between the substrate availability of arginine and citrulline and the NOS enzyme complex (Figure 5.1). Disturbances in arginine and citrulline have already been associated with an impaired fracture healing resulting in nonunion in humans ${ }^{11}$. However, the pathogenesis has not been elicited yet.

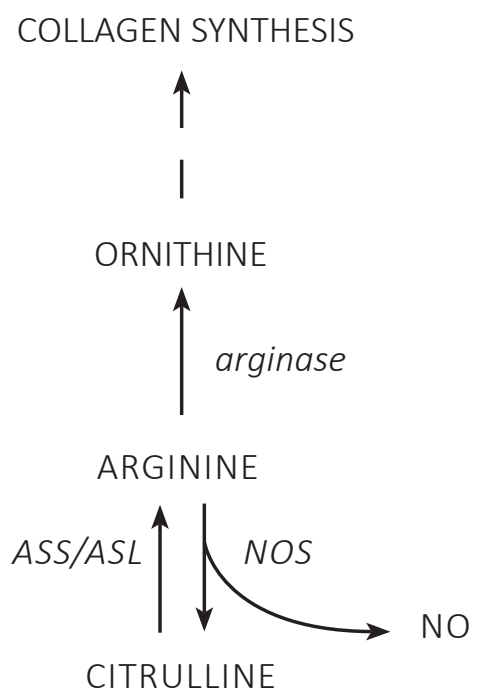

Figure 5.1. Schematic representation of arginine metabolism. Arginine can be converted into ornithine by the enzyme arginase. Ornithine is a precursor of collagen. Arginine can also be converted into citrulline by one of the nitric oxide synthases (NOS); neuronal NOS1, inducible NOS2 or endothelial NOS3. During this process the free radical nitric oxide (NO) is produced. Citrulline can be converted back into arginine by the enzymes ASS (argininosuccinate synthase) and ASL (argininosuccinate lyase). 
In vivo studies in rats showed the presence and localization of NOS isoforms in callus samples after creation of a femoral fracture. mRNA and protein activity of inducible NOS (iNOS or NOS2) was present during the first phase of fracture repair and was mainly localized within the intramembranous region along the edge of the periosteal callus. The constitutive and calcium dependent endothelial (eNOS or NOS3) and neuronal NOS (nNOS or NOS1) were found in later stages of fracture healing and mainly in cells lining blood vessels and in the fibrochondral region between fibrous tissue and cartilage respectively ${ }^{12-14}$.

We hypothesized that low amounts of NO and disturbances in arginine substrate metabolism due to an absence of either the Nos 2 or Nos 3 gene will inhibit callus formation and increase the risk of nonunion. Therefore, we studied the formation of callus and the arginine metabolism after a femur osteotomy with periosteal cauterization in a mouse model of delayed union.

\section{MATERIALS AND METHODS}

\section{Animals and surgical procedure}

In this study, skeletally mature, 20 to 24 week old specific pathogen free (SPF), female C57Bl6/J (RCC Switzerland) and Nos $2^{--}$and $\mathrm{Nos}^{-/-}$, both backcrossed more than 10 generation into the $\mathrm{C} 57 \mathrm{Bl} / \mathrm{J}$ background, with constructs previously described by Laubach et $a l^{15}$ and Shesely et $a l^{16}$ respectively (kindly provided by Dr. Theo Hakvoort, University of Amsterdam, The Netherlands), mice were used. All mice were housed in groups of five in individually ventilated cages (IVC) with a 12-hour day-night cycle. Mice were fed standard diet (3436, Prowimi, Switzerland) and water ad libitum. All animals were allowed to acclimatize for 2 weeks prior to surgical intervention. After these 2 weeks, mice were randomly assigned to the micro-computed tomography ( $\mathrm{N}=9$ /group), the amino acid and RNA analysis ( $N=6 /$ group) or the histology ( $N=9$ /group) groups for analysis. See table 5.1 for a complete overview of animals per mouse strain, group of analysis and days of follow-up.

Anaesthesia was induced by placing the mice in an induction box flooded with isoflurane (Isoflurane, Baxter AG, Switzerland). For intraoperative analgesia, $0.1 \mathrm{mg} / \mathrm{kg}$ s.c. buprenorphine (Temgesic, Essex Chemie AG, Switzerland) was administered. During surgery, animals were kept under 1.5-2 \% isoflurane inhalation anaesthesia and on a heating pad to prevent hypothermia. After aseptic preparation of the surgical field, animals were placed in prone position and a lateral skin incision starting at the base of the tail towards the left knee was made. By blunt dissection between the quadriceps and biceps femoris muscles, the femur was exposed and a $1 \mathrm{~mm}$ segment of periosteum was cauterized 
circumferentially during 0.5 seconds. The soft tissue was protected by a Teflon foil during cauterization. Thereafter, an internal plate ${ }^{17}(7 \times 1.5$ x 0.7 mm, MouseFix, RISystems Davos, Switzerland) was placed on the femur and after predrilling with a $0.33 \mathrm{~mm}$ drill bit the plate was fixed with four angular stable MouseFix screws (2.0 $\mathrm{mm}$ in length). Following fixation, a $0.45 \mathrm{~mm}$ mid-diaphyseal femoral gap osteotomy was performed with a Gigli wire saw in the center of the cauterized segment. Each screw was untightened by half a turn to induce secondary fracture healing ${ }^{18}$. Fascia and skin were closed in routine fashion (5-0 Vicryl Rapide, Ethicon and Proline, Ethicon, Belgium). At the end of surgery, plate placement and fixation was confirmed radiographically. In the following 48 hours, mice received $0.1 \mathrm{mg} / \mathrm{kg}$ s.c. buprenorphine every $10-14$ hours and for the first 5 days postoperatively $8 \mathrm{mg}$ paracetamol per os/mouse/day was given through the drinking water (Dafalgan, Upsamedica, Switzerland).

Mice were sacrificed using $\mathrm{CO}_{2}$ following different periods of fracture healing ( 7 , 28 and 42 days after osteotomy). The veterinary welfare and ethics committee of the Canton of Graubünden (Switzerland) approved the experimental set-up and procedures of this study (permit number GR 23/2006).

\section{Amino acid measurements}

To determine arginine, citrulline and ornithine concentrations, blood was collected post mortem in heparinized tubes on ice for amino acid measurements and centrifuged immediately ( $4{ }^{\circ} \mathrm{C}, 15 \mathrm{~min}$ at $8,500 \mathrm{~g}$ ) to obtain plasma. For amino acid analysis, plasma was deproteinized using acetonitrile (ratio plasma : acetonitrile $1: 2$ ), vortexed and stored until further analysis at $-80{ }^{\circ} \mathrm{C}$. Tissue samples for amino acid measurements were snap frozen in liquid nitrogen directly after harvesting. Before analysis, frozen homogenized tissue samples were added to $0.1 \mathrm{~g}$ of glass beads ( $1.0 \mathrm{~mm}$ diameter) in $250 \mu \mathrm{l}$ of $5 \%$ sulfosalicylic acid for deproteinization, beaten for 30 seconds at maximum speed with mini-beadbeater (BioSpec Products, Bartlesville, Oklahoma, USA) and stored at $-80 \stackrel{\circ}{ } \mathrm{C}$ until further analysis. The contralateral right femurs of the mice were used as unfractured control bones. Plasma and tissue amino acid concentrations were measured by HPLC (high-performance liquid chromatography) as previously described ${ }^{19}$.

The arginine availability in plasma and callus tissue was calculated as [arginine] / ([ornithine] + [lysine]). This is based on the uptake of arginine, ornithine and lysine in cells via the $\mathrm{y}^{+}$transport system ${ }^{20,21}$.

\section{Immuno histochemistry}

Following euthanasia, internal fixators were removed from the femurs and samples were fixed in $4 \%$ buffered paraformaldehyde solution and decalcified using EDTA. Samples were embedded in paraffin and $4 \mu \mathrm{m}$ sections were prepared. For immuno 
histochemical analysis, sections were deparafinised in xylene and rehydrated from graded ethanol to water. Endogenous peroxidase activity was blocked using $0.3 \%$ hydrogen peroxide in methanol $(15 \mathrm{~min})$. Sections were incubated for 1 hour with a MPO (myeloperoxidase) primary antibody (polyclonal antibody, A0398, DakoCytomation, Glostrup, Denmark) at room temperature. Specific antibodybinding was detected with a horseradish peroxidase (HRP) labeled goat- $\alpha$-rabbit IgG antibody (Jackson Immunoresearch, Westgrove, PA, USA). Visualization of the staining was performed with 3-amino-9-ethylcarbazole (AEC) and followed by nuclear counter staining with haematoxylin (Sigma, St. Louis, MO, USA). Scoring of MPO was done on 3 separate locations on the section (proximal part, distal part and callus) by two independent, blinded, researchers and on a three point scale (0: no MPO detectable, 1: intermediate signal and 2: strong signal).

\section{Histology}

$4 \mu \mathrm{m}$ sections were used after fixation and decalcification as described above. Sections were deparafinised in xylene and rehydrated from graded ethanol (100\%96\%-70\%) to distilled water. For morphogenetic analysis, sections were stained in hematoxylin and eosin (H\&E).

\section{RNA isolation and QPCR}

After collecting samples, tissues were snap frozen in liquid nitrogen and stored at $-80{ }^{\circ} \mathrm{C}$ until further analysis. Before RNA isolation, samples were crushed with pestle and mortar on liquid nitrogen. To isolate total RNA, crushed samples were incubated in Trizol and were beaten with glass beads thrice for $10 \mathrm{~s}$ with a mini-beadbeater (Biospec Products, Bartlesville, OK, USA). Afterwards, RNA was precipitated using isopropanol and centrifugation (30 min, 11,000 rpm, $4^{\circ} \mathrm{C}$ ). After precipitation, pellets were washed with $80 \%$ ethanol and air dried before dissolving in DEPC (diethylpyrocarbonate) treated water. Genomic DNA was removed using DNase I treatment (Promega, Madison, WI, USA). RNA was precipitated using $100 \%$ ethanol with $3 \mathrm{M} \mathrm{NaAc}$ for 30 minutes at $-80{ }^{\circ} \mathrm{C}$ before centrifugation (30 min, $11,000 \mathrm{rpm}, 4^{\circ} \mathrm{C}$ ). After washing with $80 \%$ ethanol, pellets were dissolved in $20 \mu \mathrm{LEPC}$ treated water. Standard cDNA synthesis was performed by using the iScript cDNA synthesis kit (Biorad Products, Hercules, CA, USA).

For quantitative PCR, iQ SYBR Green Supermix (Biorad Products, Hercules, CA, USA) and gene-specific Nos2, Nos3, Arg1, ActB and Ppia forward and reversed primers (see table 1) were added to the cDNA. The cDNA was amplified using the MyiQ system (Biorad Products, Hercules, CA, USA) via a 3-step program: 40 cycles of denaturation $\left(95^{\circ} \mathrm{C}, 10 \mathrm{~s}\right)$, annealing $\left(60^{\circ} \mathrm{C}, 20 \mathrm{~s}\right)$ and elongation $\left(70^{\circ} \mathrm{C}\right.$, 20s). Further analysis was performed using the MyiQ software. 
TABLE 5.1 Primer sequences for quantitative PCR

\begin{tabular}{lll}
\hline Gene & Name & Primer sequence $\left(\mathbf{5}^{\prime} \rightarrow \mathbf{3}\right.$ ') \\
\hline Arg1 & Arg1-F & GGAGAGCCTTCCTGCACTTT \\
\cline { 2 - 3 } Arg1-R & GTGCCTTGGTCTACATTGAACATAC \\
\hline \multirow{2}{*}{ Nos3 } & iNOS-F & TTGCAAGCTGATGGTCAAGATC \\
\cline { 2 - 3 } & iNOS-R & CAACCCGAGCTCCTGGAA \\
\hline ActB & eNOS-F & TTAATGTGGCCGTGTTGCA \\
\hline Ppia & B-actin-F & CTCTTGATGGAAGACAGGAGTTAGG \\
\cline { 2 - 3 } & B-actin-R & CACAGGATGCAGAAGGAGATTACTG \\
\hline CycloA-F & TTCCTCCTTTCACAGAATTATTCCA \\
\hline
\end{tabular}

Abbreviations: F: forward primer; R: reversed primer

\section{Micro-computed tomography analysis}

Femurs were scanned by micro-computed tomography ( $\mu \mathrm{CT} 40$, Scanco Medical, Switzerland) at $70 \mathrm{kV}_{\mathrm{p}}$ and $114 \mu \mathrm{A}$ with $200 \mathrm{~ms}$ integration time. The femur was positioned, so that its longitudinal axis was oriented perpendicular to the X-ray radiation. This position was maintained during the analysis by inserting a pin in the most proximal and distal screw hole, which fixed the bone. The volume between the two screw holes was measured with 400 two-dimensional transversal cross-sections in a 1024 x 1024 pixel matrix, with a spatial resolution of $12 \mu \mathrm{m}$. After selection of the regions of interest (ROI), a Gaussian filter (sigma 0.8, support 1) was used for a partial suppression of the noise. Based on histogram of attenuation distribution, tissue was segmented into woven bone (low degree of mineralization; 14.5-36\% of maximal image gray value) and lamellar bone (high degree of mineralization $>36 \%$ ). Based on the described gray values, the degree of mineralization could be quantified ${ }^{18}$.

Four regions of interest were described. The first was the total region (TOT) and was defined as the complete region between the most proximal and distal placed screws. The second, periosteal region (PER) contained the complete volume of newly formed bone tissue within the original outer cortical border of the femur. The endosteal (END), third, region contained the volume of newly formed bone within the inner cortical border (i.e. within the bone marrow cavity). The final region of interest was the actual fracture gap (GAP) itself. It was defined as the space between the proximal and distal fracture part with exclusion of original cortical structure and bone fragments. The regions of interest are represented schematically in figure 6A.

\section{Statistical analysis}

Statistical analysis was performed using GraphPad Prism 5 (GraphPad, San Diego, California, USA). Normality was checked using the Shapiro-Wilks test. The data 
are represented as means and standard error of the mean (SEM). Significance was tested using ANOVA with post hoc Bonferroni correction. P-values below 0.05 were considered statistical significant.

\section{RESULTS}

\section{Increased plasma amino acid concentrations in Nos3 deficient mice}

No differences were present in systemic concentrations of arginine, citrulline and ornithine between wild type mice and both NOS knock-out groups in plasma samples obtained after 7 days of fracture healing (Figure 5.2A, 5.2B and 5.2C).

After 28 days of fracture healing, plasma concentrations of all amino acids were significantly enhanced in $\mathrm{Nos}^{-/-}$mice compared with wild type animals (arginine: $\mathrm{p}<0.001$; Figure 5.2A, citrulline: $\mathrm{p}<0.0001$; Figure 5.2B, ornithine: $\mathrm{p}<0.05$; Figure 5.2C). Arginine ( $\mathrm{p}<0.05$; Figure 5.2A) and citrulline $(\mathrm{p}<0.0001$; Figure 5.2B) concentrations of $\mathrm{Nos}^{-/-}$mice also showed significant higher concentrations when compared with Nos $2^{--}$animals. Measured citrulline levels showed a distinct decrease $(\mathrm{p}<0.01)$ in $N o s 2^{--}$mice between samples measured at 7 and 28 days of follow-up, whereas in NOS3 deficient animals these concentrations were enhanced $(\mathrm{p}<0.05)$.

The arginine availability in plasma, defined by the ratio between the arginine concentration and the combined ornithine and lysine concentration decreased over time between samples obtained after 7 and 28 days of fracture healing in NOS2 and NOS3 deficient animals (Figure 5.2D).

\section{Decreased amino acid concentrations in NOS2 and NOS3 deficient callus tissue}

Amino acid concentrations in femur tissue were measured in fractured bones and contralateral unfractured control bones. Arginine concentrations measured in callus tissue showed no differences between the different mouse strains at both time points (Figure 5.3A).

After 28 days of fracture healing, callus tissue from wild type mice showed significant higher citrulline concentrations when compared to callus tissue from 7 days of fracture healing ( $\mathrm{p}<0.05$; Figure 5.3B). Also, NOS2 deficient mice had significantly lower citrulline concentrations compared to wild type animals at 28 days $(\mathrm{p}<0.01)$. Callus citrulline concentrations in Nos3 knockout mice tended to be lower compared to wild type mice.

After 28 days of fracture repair, callus tissue of wild type and NOS3-deficient mice showed $\sim 1.5-2$ fold lower ornithine concentrations when compared to callus tissue collected after 7 days ( $p<0.001$ and $p<0.05$ respectively; Figure 5.3C). 
Fractured femurs in wild type mice after 28 days showed significant lower ornithine concentrations when compared with Nos2 $2^{- \text {mice }}(\mathrm{p}<0.05)$. Fractured femurs of NOS2 as well as NOS3 deficient mice showed significantly higher ornithine concentrations when compared with their contralateral non-fractured femurs after 7 days of fracture healing ( $\mathrm{p}<0.05$ and $\mathrm{p}<0.01$ respectively, figure 5.3C). The decrease of ornithine concentrations over time as visible in fractured femurs was not present in unfractured controls.

A

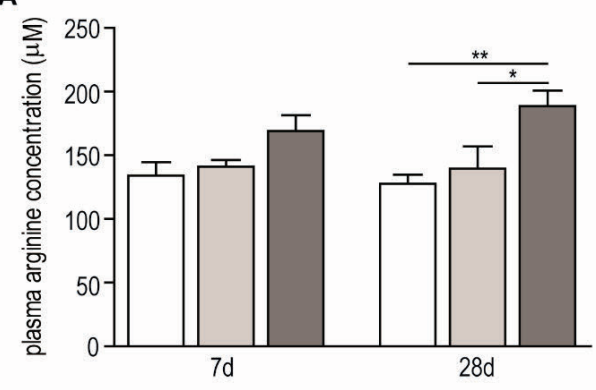

C

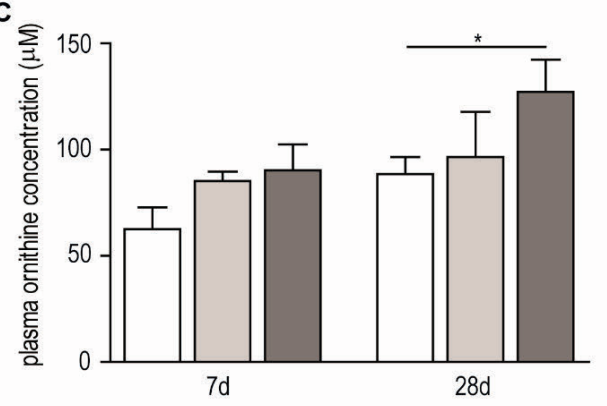

B

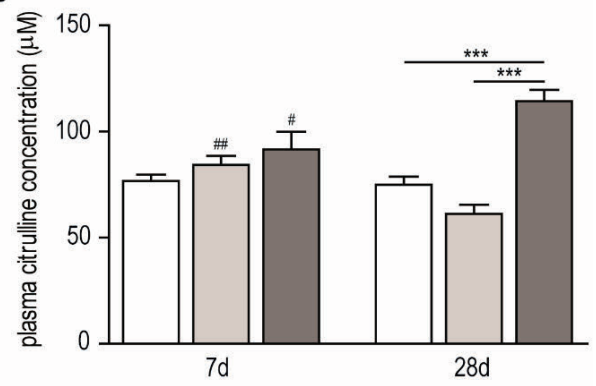

5

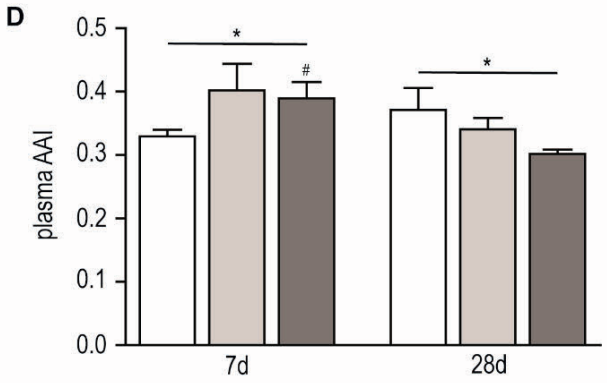

$\square$ WT $\square$ Nos2 $^{*-} \square$ Nos3 $^{*}$

Fig. 5.2. Plasma concentrations $(\mu \mathrm{M})$ of arginine (panel A), citrulline (B) and ornithine (C) in mice after 7 and 28 days of fracture healing in wild type (white), Nos2 (light grey) and $\mathrm{Nos}^{2 /}$ (dark grey) mice. Plasma arginine availability index (AAI, panel D) was calculated as the ratio between the arginine concentration and combined ornithine and lysine concentrations. Significance levels between mouse strains: *: $\mathrm{p}<0.05 ;{ }^{* *}: \mathrm{p}<0.001{ }^{* * *}: \mathrm{p}<0.0001$. Significance levels between time points: \#: $\mathrm{p}$ $<0.05 ; \#$ : $\mathrm{p}<0.01$.

Callus tissue of wild type animals after 28 days of fracture healing showed significantly higher arginine availability (Figure 5.3D) when compared with both Nos2 $^{--}(\mathrm{p}<0.001)$ and Nos3 $^{-/}(\mathrm{p}<0.05)$ mice. The tissue arginine availability index increased between 7 and 28 days of follow-up in both wild type mice ( $\mathrm{p}$ $<0.0001)$ and NOS3-deficient mice $(\mathrm{p}<0.05)$, but not in Nos 2 knockout mice. 


\section{Elevated myeloperoxidase influx in callus tissue of NOS2 and NOS3 deficient mice}

Femur samples of both NOS2 and NOS3 deficient animals collected 28 days after the osteotomy procedure showed significantly elevated neutrophil influx ( $\mathrm{p}<$ 0.05 and $\mathrm{p}<0.01$ respectively; Figure $5.4 \mathrm{~A}$ and $\mathrm{B}$ ) as measured by MPO levels in the callus region and the proximal and distal marrow cavities when compared to wild type mice. NOS3 deficient mice regained a normal level of MPO influx after 42 days of fracture healing, whereas Nos 2 knock-out animals still showed a high degree of MPO influx ( $\mathrm{p}<0.01$ when compared with wild type animals).

Figure 5.4C shows H\&E staining results of fractured femurs after 42 days of followup. Wild type animals presented with callus formation between the proximal and distal fracture parts, whereas in both $\mathrm{Nos}_{2}{ }^{--}$and $\mathrm{Nos}^{-/-}$mice, no evident callus formation was visible.
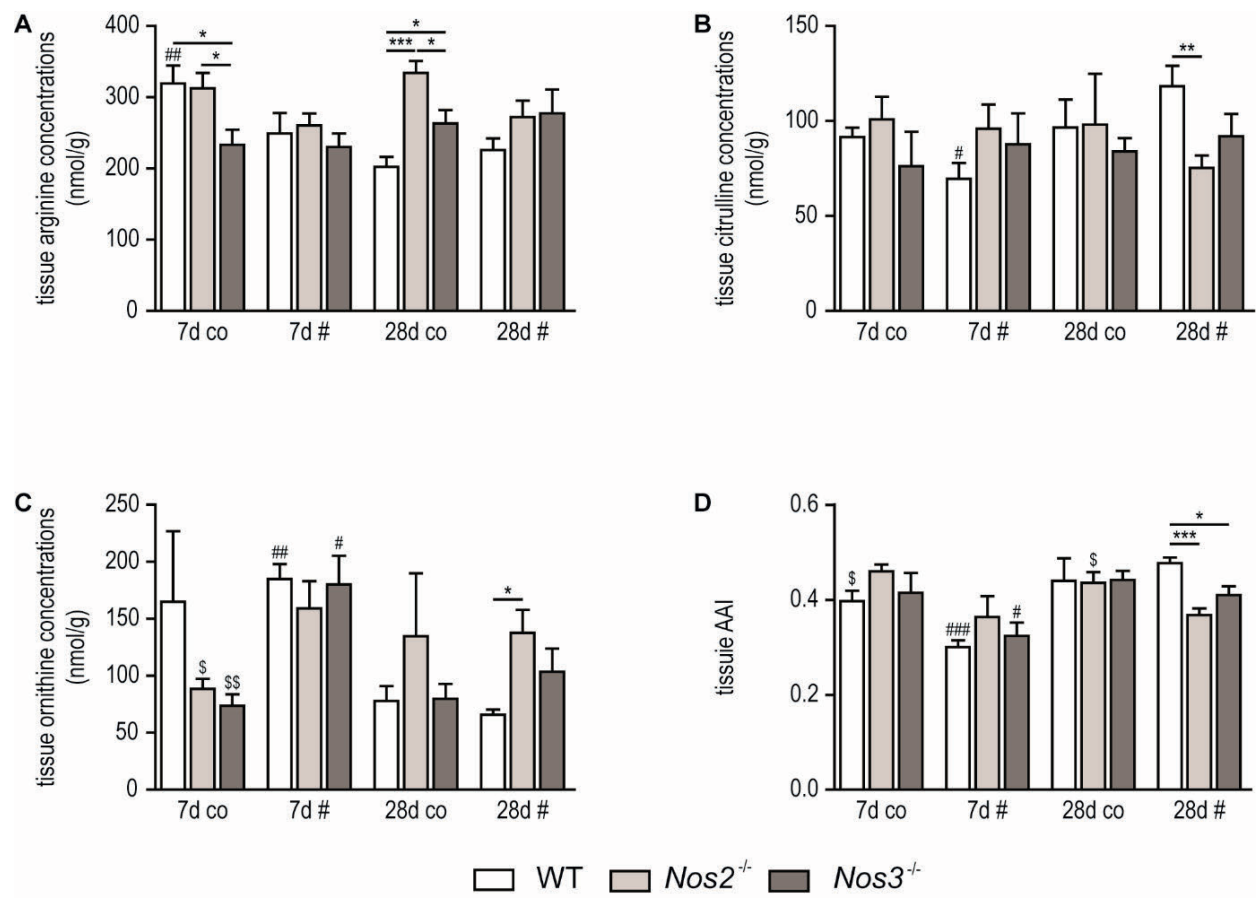

Fig. 5.3. Arginine (panel A), citrulline (B) and ornithine (C) concentrations in femoral callus tissue in $\mathrm{nmol} / \mathrm{g}$, measured after 7 and 28 days of fracture healing in osteotomized (\#) and contralateral

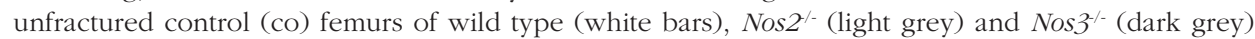
mice. Arginine availability index (panel D, AAI) was calculated as the ratio between the arginine concentration and combined ornithine and lysine concentrations. Significance levels between mouse strains: *: $\mathrm{p}<0.05 ;{ }^{* *}: \mathrm{p}<0.01$; $^{* * *}: \mathrm{p}<0.001$. Significance levels between time points: \#: $\mathrm{p}<0.05$; \#\#: $\mathrm{p}<0.01 ; \# \#: \mathrm{p}<0.0001$. Significance between control and fractured femurs: $\$: \mathrm{p}<0.05$ and $\$ \$$ : $\mathrm{p}<0.01$. 
A

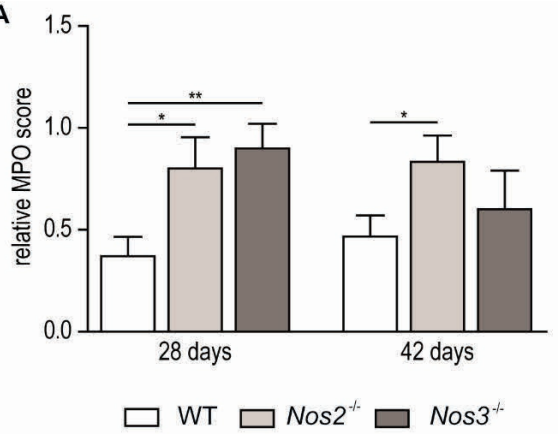

B

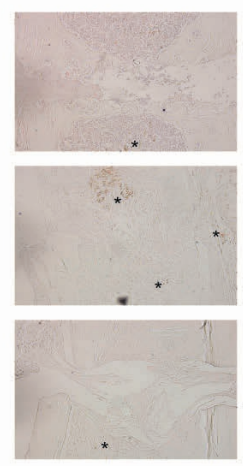

28 days
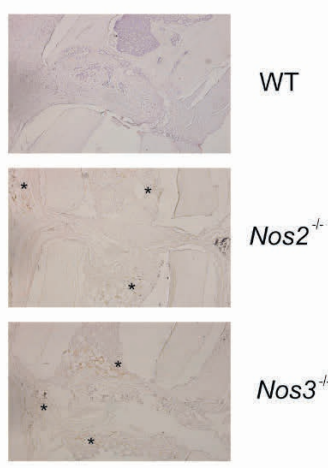

42 days

C

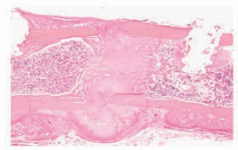

WT

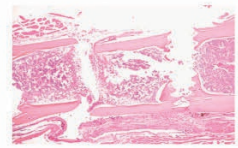

Nos $2^{-1-}$

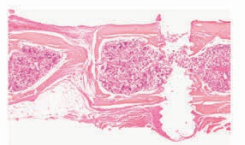

Nos $3^{-1}$

Fig. 5.4. Relative myeloperoxidase (MPO) score as scored by 2 independent blinded researchers on 3 anatomical locations (proximal and distal marrow cavity and callus) in murine femurs at 28 and 42

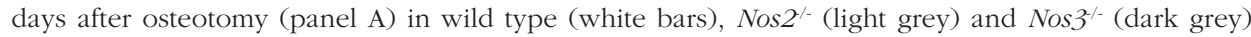
mice. *: $\mathrm{p}<0.05 ;^{* *}: \mathrm{p}<0.01$. Panel B shows representative qualitative MPO stainings of each group. Asterixes in panel B indicate MPO staining. Panel C shows H\&E staining of fractured femurs after 42 days of fracture healing of wild type and both NOS deficient groups of animals.

\section{Upregulation of arginase-1 RNA in callus tissue}

Contralateral unfractured control femurs showed no Arginase-1 expression after both 7 and 28 days of follow-up (Figure 5.5A), while all fractured femurs presented detectable levels of Arginase-1. In addition, in all mice, Arginase-1 showed a 2 -fold lower concentration at 28 days compared to 7 days. Furthermore, at 28 days NOS3-deficient mice had a significantly lower Arginase-1 expression compared to NOS2 depleted mice $(\mathrm{p}<0.05)$.

\section{Nos 2 and Nos3 expression}

Nos 2 mRNA expression was lower in the fractured femur at 7 days compared to the normal femur, while at 28 days the reverse was present. While Nos 2 expression was absent in NOS2-deficient mice, Nos2 expression in NOS3-deficient mice was higher at 28 days (Figure 5.5B, p $<0.05$ ).

Nos3 expression in wild type animals remained similar in fractured femur tissue at 7 and 28 days compared to non-fractured femurs. Nos 3 expression at 28 days tended to be increased in Nos 2 knockout mice compared to wild type and Nos 3 knockout mice (Figure 5.5C). 
A

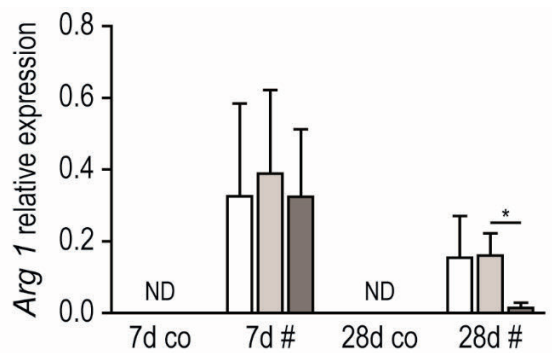

C

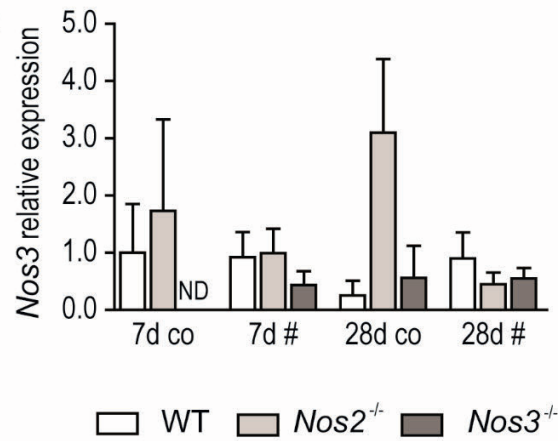

B

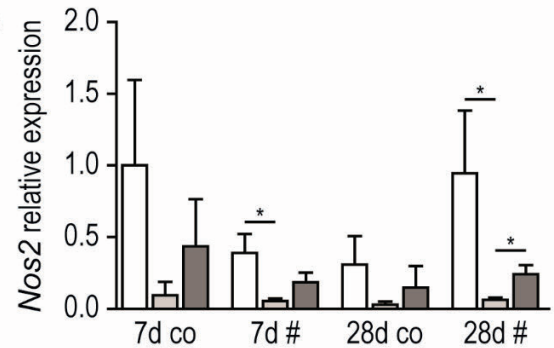

Fig. 5.5. Relative RNA expression of Arginase-1 (Arg-1, panel A), inducible nitric oxide synthase (Nos2, B) and endothelial nitric oxide synthase (Nos3, C) measured in femoral callus tissue after 7 and 28 days of fracture healing in osteotomized (\#) and contralateral unfractured control (co) femurs of

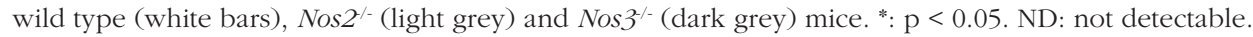

\section{Evident nonunion development in $\mathrm{Nos}^{-/-}$and $\mathrm{Nos}^{-/}$on micro-computed tomography}

In figure 5.6B-G, qualitative images of representative micro-CT measurements are shown of femurs after 28 and 42 days of fracture repair in wild type mice, and Nos 2 and Nos3 knockout mice. Grey structures indicate mineralized bone and green structures indicate newly formed callus tissue. Both NOS deficient groups of animals showed almost no signs of bone formation at both time points.

Micro-CT measurements of total callus volume showed higher volumes in wild type mice when compared to both NOS deficient strains of animals (Figure $5.6 \mathrm{H}$ ) after 28 and 42 days of fracture healing ( $<$ 0.05). Periosteal callus volume (Figure 5.6I) was significantly lower in Nos 2 knockout mice when compared with wild types $(\mathrm{p}<0.05)$ after 28 days of fracture healing. Endosteal callus volumes were significantly lower in Nos3 knockout mice after 28 days of fracture healing (Figure $6 \mathrm{~J}, \mathrm{p}<0.05)$. After 42 days of fracture healing, NOS2 and NOS3 deficient animals both showed lower periosteal and endosteal callus volumes in comparison with wild type mice $(\mathrm{p}<0.05)$.

In the GAP region between the proximal and distal part of the femur, $3-4$ fold lower callus volumes were observed in both NOS deficient strains when compared 
to wild type mice after 28 and 42 days of fracture healing. However, due to the large variation in the quantified results, differences did not reach a level of significance (figure $5.6 \mathrm{~K}$ ).
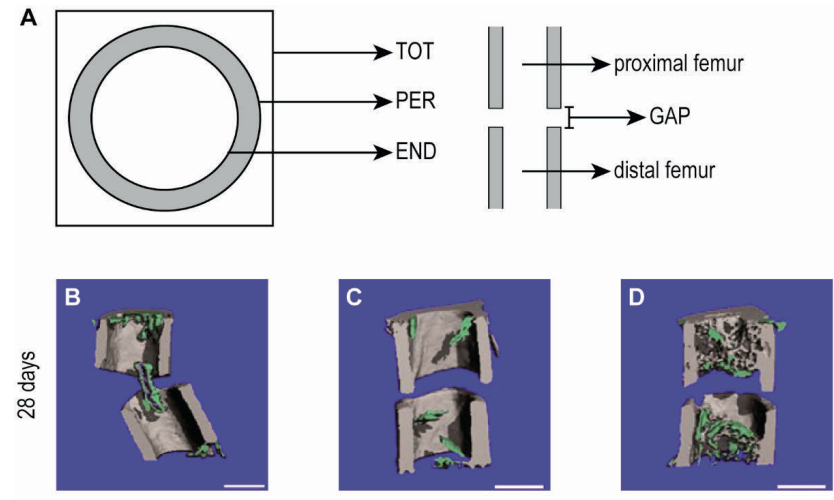

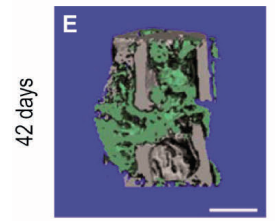

wild type

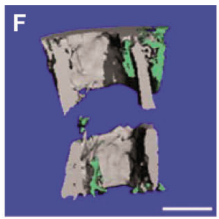

Nos2 KO

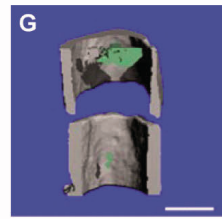

Nos3 KO

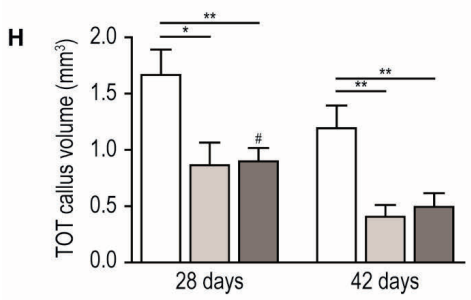

I
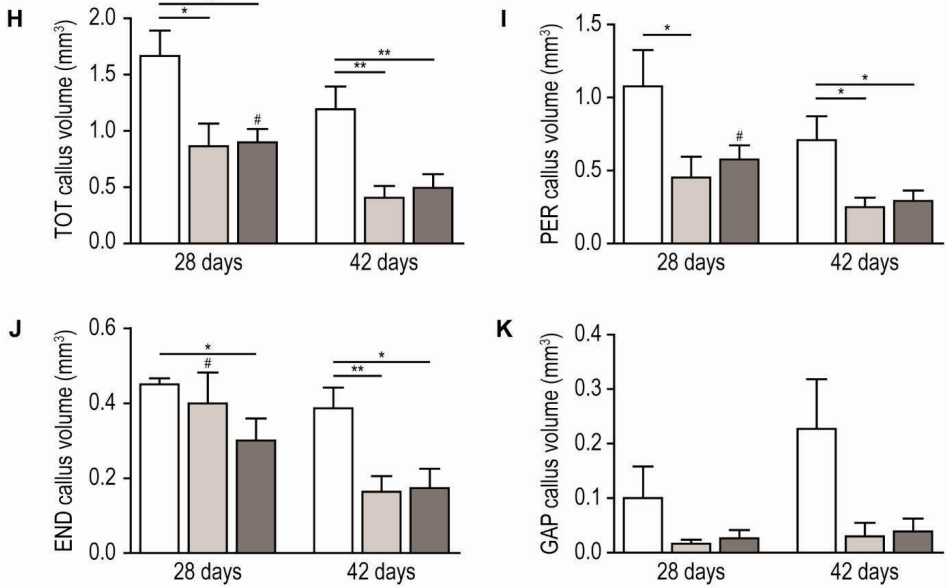

K

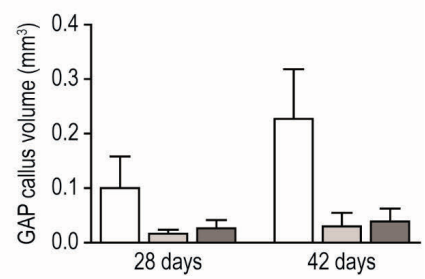

\section{WT $\square$ Nos2 $^{-*} \square$ Nos3 $^{-+}$}

Fig. 5.6. Panel A shows a schematic representation of the four different regions of interest measured with $\mu \mathrm{CT}$ : TOT: Total region between de most proximal and distal screw, PER: periosteal region, END: endosteal region and GAP: the part between the proximal and distal part of the femur where the osteotomy was performed. Panels B to G show qualitative images of the different groups. (B): wild

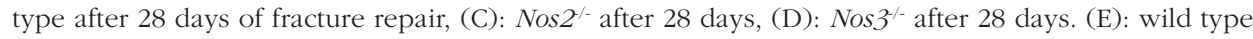


after 42 days, (F): Nos $2^{--}$after 42 days and (G): Nos $3^{-/}$after 42 days. Grey structures indicate bone tissue, green represents developed callus tissue. Scale bars indicate a length of $1 \mathrm{~mm}$.

Callus volume (in $\mathrm{mm}^{3}$ ) after $\mu \mathrm{CT}$ measurement of four different regions of interest: panel H: TOT, panel I: PER, panel J: END and panel K: GAP. White bars represent wild type mice, light grey Nos2 ${ }^{\prime}$ and dark grey Nos3 ${ }^{-1}$. * $: \mathrm{p}<0.05$ (difference between mouse strains), ${ }^{* * *}: \mathrm{p}<0.01 ; \#: \mathrm{p}<0.05$ (difference between time points).

\section{DISCUSSION}

In the present study we show that both the absence of either endothelial or inducible nitric oxide synthase results in an inadequate fracture healing, as demonstrated by the transition of a delayed union into a nonunion in mice after femur osteotomy with periosteal cauterization. Both NOS2- and NOS3-deficient mice also exhibited a prolonged increase of neutrophil influx in the later stages of fracture repair, indicating that a disturbed inflammatory response plays a role in the development of nonunion. Finally, deletion of the Nos 2 and Nos 3 gene induced a disturbed systemic and local arginine-NO metabolism.

During normal conditions, the process of bone healing in mice until there is no interfragmentary motion takes around three weeks ${ }^{22}$. In our study, we cauterized the periosteum to create a clinically relevant situation of compromised fracture healing. This cauterization delayed the fracture healing in wild type animals by approximately one week (data not shown).

As far as our knowledge extends, our study is the first to measure callus volume in both Nos 2 and Nos3 knockout animals in comparison with wild type animals during different time points in the fracture healing process. Micro-CT is an ideal method to not only quantify tissue volumes but also to differentiate it spatially. We were able to show that NOS2 and NOS3-deficient mice had significantly lower quantities of total callus volume, and thus the important influence of these enzymes on fracture healing. Whereas in NOS2-deficient animals periosteal callus formation was hampered after 28 days of fracture healing, Nos 3 knockouts showed significantly lower endosteal callus volume, suggesting different pathways in which fracture healing is disturbed in these animals.

Diwan et al and Zhu et al both showed a temporal ${ }^{13}$ and localized ${ }^{14}$ increased expression of NOS isoforms, compared to the normal unfractured femoral cortex ${ }^{12}$, during the normal healing process of femoral fractures in rodents. In their studies, NOS2 was primarily present during the initial inflammatory stage and mainly found within the intramembranous region, along the edge of the periosteal callus. NOS3 is present during the secondary bone formation in cells in the chondral region and lining of blood vessels. 
In our study, a decrease in NOS2 expression in the callus tissue after 7 days of delayed fracture healing was present in wild type animals compared to normal non-fractured femurs, while the opposite occurred after 28 days. In a study by Corbett et $a l^{23}$ levels of NOS2 protein displayed a similar pattern at 7 and 28 days of fracture healing. Thus, while in normal fracture healing NOS2 activity is upregulated, delayed fracture healing coincides with a decreased expression of NOS2 compared to normal bone. Indeed, with complete deletion of the NOS2 expression we demonstrate that bone healing is further compromised and nonunion occurs. This relationship is further emphasized in studies were suppression of NO synthesis by feeding rats orally with the non-selective NOS inhibitor L-nitroso-arginine methyl ester (L-NAME) resulted in a decrease of callus cross-sectional area and maximum failure load during three-point-bending tests ${ }^{12}$. Fractured femurs of Nos 2 knockout mice are known to have a decreased maximum energy absorption and torsional failure during strength testing ${ }^{24}$. Biomechanical properties of unfractured Nos 2 knockout femurs showed no differences when compared to those of wild type mice. These data emphasize the importance of NOS2 in fracture healing.

Our results demonstrate that in the absence of NOS3, fracture healing is hampered with decreased callus formation and nonunion development. Previously, an increased expression of NOS3 was shown in cortical blood vessels during fracture repair, which mediates an increased blood flow during normal fracture repair ${ }^{23}$. An experimental model of normal fracture healing in rats showed changes in local vascular reactivity at the fracture site after intravenous pharmacological stimulation or inhibition of NO during initial phases of fracture healing ${ }^{8}$. NOS3 is known to play a key role in postnatal regulation of bone mass, as young Nos3 knockout mice show a reduced bone volume and defects in osteoblast differentiation, maturation and activity and reduced rates of growth factors ${ }^{25,26}$. These data suggest the importance of NOS3 in the later bone formation phases.

As previously reported, the inflammatory response plays an important role in normal fracture healing ${ }^{1,27}$. In our study, we showed a higher level of MPO in callus tissue and proximal and distal marrow cavities in the femur of both NOS2 and NOS3-deficient animals at 28 days, while it continued to be increased until 42 days in the NOS2-deficient fracture callus. NOS2 expression is known to be drastically increased on RNA and protein levels in osteoblasts and bone marrow macrophages during inflammatory conditions ${ }^{28}$. In addition, Watanuki et al demonstrated inadequate response of bone and bone marrow cells to reloading of unloaded NOS2 deficient murine tibiae ${ }^{29}$.

In this study, we have not looked into the role of NOS1 during the follow-up period. NOS1 is mainly up-regulated in the later stages of fracture healing ${ }^{13}$ (i.e. bone remodeling), hence after callus formation. The fact that nonunion development 
depends on disturbances during the inflammatory phase and subsequent callus formation ${ }^{30,31}$ led us to focus on NOS2 and NOS3 which is generally expressed during the primary phases of bone healing.

In a previous study, our group already showed that disturbed amino acids concentrations were associated with nonunion development in humans ${ }^{11}$. In atrophic nonunions, concentrations of arginine, citrulline and ornithine were significantly lower in comparison with healthy controls. To determine the role of the substrate availability (arginine and citrulline) on delayed union and nonunion development in this model, these amino acid concentrations were measured. As observed, the arginine availability was significantly decreased after 28 days of fracture healing in Nos 2 and Nos 3 knock-out animals, which indicates the importance of NOS presence and arginine availability on fracture healing. In this study, arginine availability was significantly decreased. Kdolsky et al were the first to report a possible influence of oral L-arginine supplementation to be beneficial on fracture healing in an in vivo study in guinea pigs ${ }^{32}$ with signs of improved fracture healing on radiographic imaging. Based on these findings and this study, we hypothesize that stimulation of the arginine-NO metabolism might be a promising possible therapeutic option in decreasing the percentage of nonunion and delayed union development after fractures.

In conclusion, this study shows that a disturbed arginine-NO metabolism by blocking inducible or endothelial NOS facilitates the development of nonunion in a delayed union mouse model. 


\section{REFERENCES}

1. Schindeler A, McDonald MM, Bokko P, Little DG. Bone remodeling during fracture repair: The cellular picture. Semin Cell Dev Biol 2008; 19(5): 459-66.

2. Mills LA, Simpson AH. The relative incidence of fracture non-union in the Scottish population (5.17 million): a 5-year epidemiological study. BMJ Open 2013; 3(2).

3. Brighton CT, Shaman P, Heppenstall RB, Esterhai JL, Jr., Pollack SR, Friedenberg ZB. Tibial nonunion treated with direct current, capacitive coupling, or bone graft. Clin Orthop Relat Res 1995; (321): 223-34.

4. Frolke JP, Patka P. Definition and classification of fracture non-unions. Injury 2007; 38 Suppl 2: S19-22.

5. Jahagirdar R, Scammell BE. Principles of fracture healing and disorders of bone union. Surgery 2009; 27(2): 63-9.

6. Baldik Y, Talu U, Altinel L, Bilge H, Demiryont M, Aykac-Toker G. Bone healing regulated by nitric oxide: an experimental study in rats. Clin Orthop Relat Res 2002; (404): 343-52.

7. Chae HJ, Park RK, Chung HT, et al. Nitric oxide is a regulator of bone remodelling. J Pharm Pharmacol 1997; 49(9): 897-902.

8. Corbett SA, McCarthy ID, Batten J, Hukkanen M, Polak JM, Hughes SP. Nitric oxide mediated vasoreactivity during fracture repair. Clin Orthop Relat Res 1999; (365): 247-53.

9. Vittur F, Lunazzi G, Moro L, et al. A possible role for polyamines in cartilage in the mechanism of calcification. Biochim Biophys Acta 1986; 881(1): 38-45.

10. Xia W, Szomor Z, Wang Y, Murrell GA. Nitric oxide enhances collagen synthesis in cultured human tendon cells. Journal of orthopaedic research : official publication of the Orthopaedic Research Society 2006; 24(2): 159-72.

11. Wijnands KA, Brink PR, Weijers PH, Dejong $\mathrm{CH}$, Poeze M. Impaired fracture healing associated with amino acid disturbances. Am J Clin Nutr 2012; 95(5): 1270-7.

12. Diwan AD, Wang MX, Jang D, Zhu W, Murrell GA. Nitric oxide modulates fracture healing. J Bone Miner Res 2000; 15(2): 342-51.

13. Zhu W, Diwan AD, Lin JH, Murrell GA. Nitric oxide synthase isoforms during fracture healing. $J$ Bone Miner Res 2001; 16(3): 535-40.

14. Zhu W, Murrell GA, Lin J, Gardiner EM, Diwan AD. Localization of nitric oxide synthases during fracture healing. J Bone Miner Res 2002; 17(8): 1470-7.

15. Laubach VE, Shesely EG, Smithies O, Sherman PA. Mice lacking inducible nitric oxide synthase are not resistant to lipopolysaccharide-induced death. Proceedings of the National Academy of Sciences of the United States of America 1995; 92(23): 10688-92.

16. Shesely EG, Maeda N, Kim HS, et al. Elevated blood pressures in mice lacking endothelial nitric oxide synthase. Proceedings of the National Academy of Sciences of the United States of America 1996; 93(23): 13176-81.

17. Matthys R, Perren SM. Internal fixator for use in the mouse. Injury 2009; 40 Suppl 4: S103-9.

18. Grongroft I, Heil P, Matthys R, et al. Fixation compliance in a mouse osteotomy model induces 
two different processes of bone healing but does not lead to delayed union. J Biomech 2009; 42(13): 2089-96.

19. van Eijk HM, Rooyakkers DR, Deutz NE. Rapid routine determination of amino acids in plasma by high-performance liquid chromatography with a 2-3 microns Spherisorb ODS II column. J Chromatogr 1993; 620(1): 143-8.

20. Morris CR, Poljakovic M, Lavrisha L, Machado L, Kuypers FA, Morris SM, Jr. Decreased arginine bioavailability and increased serum arginase activity in asthma. American journal of respiratory and critical care medicine 2004; 170(2): 148-53

21. Hong SK, Maltz BE, Coburn LA, et al. Increased serum levels of L-arginine in ulcerative colitis and correlation with disease severity. Inflammatory bowel diseases 2010; 16(1): 105-11.

22. Garcia P, Histing T, Holstein JH, et al. Rodent animal models of delayed bone healing and nonunion formation: a comprehensive review. European cells \& materials 2013; 26: 1-12; discussion -4 .

23. Corbett SA, Hukkanen M, Batten J, McCarthy ID, Polak JM, Hughes SP. Nitric oxide in fracture repair. Differential localisation, expression and activity of nitric oxide synthases. J Bone Joint Surg Br 1999; 81(3): 531-7.

24. Baldik Y, Diwan AD, Appleyard RC, Fang ZM, Wang Y, Murrell GA. Deletion of iNOS gene impairs mouse fracture healing. Bone 2005; 37(1): 32-6.

25. Aguirre J, Buttery L, O'Shaughnessy M, et al. Endothelial nitric oxide synthase gene-deficient mice demonstrate marked retardation in postnatal bone formation, reduced bone volume, and defects in osteoblast maturation and activity. Am J Pathol 2001; 158(1): 247-57.

26. Armour KE, Armour KJ, Gallagher ME, et al. Defective bone formation and anabolic response to exogenous estrogen in mice with targeted disruption of endothelial nitric oxide synthase. Endocrinology 2001; 142(2): 760-6.

27. Claes L, Recknagel S, Ignatius A. Fracture healing under healthy and inflammatory conditions. Nat Rev Rheumatol 2012; 8(3): 133-43

28. Helfrich MH, Evans DE, Grabowski PS, Pollock JS, Ohshima H, Ralston SH. Expression of nitric oxide synthase isoforms in bone and bone cell cultures. J Bone Miner Res 1997; 12(7): 1108-15.

29. Watanuki M, Sakai A, Sakata T, et al. Role of inducible nitric oxide synthase in skeletal adaptation to acute increases in mechanical loading. J Bone Miner Res 2002; 17(6): 1015-25.

30. Bastian O, Pillay J, Alblas J, Leenen L, Koenderman L, Blokhuis T. Systemic inflammation and fracture healing. Journal of leukocyte biology 2011; 89(5): 669-73.

31. Reikeras O, Shegarfi H, Wang JE, Utvag SE. Lipopolysaccharide impairs fracture healing: an experimental study in rats. Acta orthopaedica 2005; 76(6): 749-53

32. Kdolsky RK, Mohr W, Savidis-Dacho H, et al. The influence of oral L-arginine on fracture healing: an animal study. Wien Klin Wochenschr 2005; 117(19-20): 693-701. 



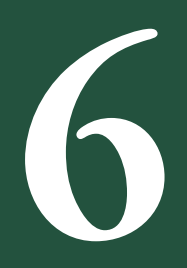
Arginine availability in reamed
intramedullary aspirate as predictor
of outcome in nonumion healing

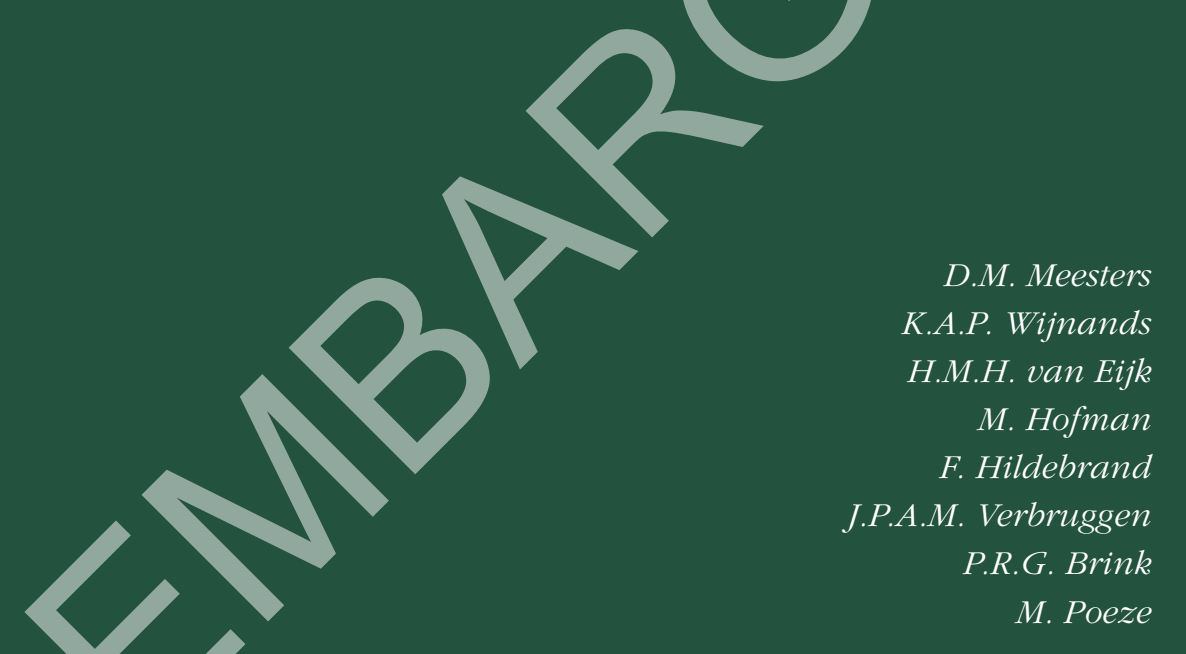

submitted 


\title{
Enhancement of fracture healing after citrulline supplementation in mice
}

\author{
D.M. Meesters \\ P.F.W. Hannemann \\ H.M.H. van Eijk \\ V.T.J. Schriebl \\ P.R.G. Brink \\ M. Poeze \\ K.A.P. Wijnands
}

European Cells \& Materials

2020 


\section{ABSTRACT}

\section{Background}

Around $10 \%$ of long bone fractures show inadequate bone healing resulting in nonunion development. A deregulated arginine-citrulline-nitric oxide metabolism caused by a poor nutritional status of the patients is a risk factor for nonunions. Additionally, previous research in mice with a disrupted arginine to citrulline conversion showed delayed healing. The study hypothesis was that stimulating said metabolism could positively influence the healing process through promotion of collagen synthesis and angiogenesis.

\section{Methods}

Adult wild type mice underwent a femur osteotomy and plate-screw osteosynthesis. Mice were randomly divided into three groups and received daily oral supplementation of arginine, citrulline or $0.9 \%$ saline (control). Body weight and food intake were measured daily. After 14 days, the mice were euthanized and femora collected. Callus formation was assessed by micro-computed tomography and concentrations of amino acids and enzymes in the femora were measured.

\section{Results}

Only citrulline-treated mice showed significantly increased bridging of the fracture gap when compared to control mice. Femur citrulline and ornithine concentrations were increased in citrulline-treated animals. qPCR showed significantly decreased expression of inflammatory markers, whereas increased expression of angiogenic and collagen-producing factors was observed in citrulline-treated mice. Altough food intake did not show any difference between the three groups, animals treated with citrulline showed a weight gain of $0.3 \mathrm{~g}$, compared with a $0.1 \mathrm{~g}$ decline in the control group.

\section{Conclusion}

Daily oral citrulline supplementation stimulated callus formation and improved the inflammatory response, positively contributing to the enhanced healing response. Finally, increased weight gain pointed towards a better post-operative recovery. 


\section{INTRODUCTION}

Every year, one percent of de general population sustains a fracture ${ }^{1,2}$. Generally, fractures heal without any complications, however, in 10\% of all long bone fractures difficulties occur during the healing process resulting in delayed union or nonunion formation ${ }^{3,4}$. Next to the fracture location, the degree of (soft) tissue injury and the type and quality of (surgical) treatment as well as several patient dependent risk factors are known which contribute to nonunion development. Besides the use of certain drugs (e.g. NSAIDs) and a disturbed vascularity (smoking, diabetes), malnutrition is one of the major risk factors for an impaired fracture healing process 5,6 .

One of the main metabolic processes influencing bone healing is the argininecitrulline-nitric oxide (NO)-metabolism ${ }^{7}$. During physiological conditions, the semi-essential amino acid arginine is produced by conversion of citrulline via the cytosolic enzymes arginine succinate lyase and arginine succinate synthethase. Arginine can be converted back into citrulline by one of the nitric oxide synthase enzymes (NOS). During the inflammatory phase of fracture healing the inducible NOS (Nos2) is active and mainly localized in the intramembranous region along the periosteal callus. During the later phases in the healing process, the constitutive and calcium dependent endothelial NOS (Nos3) and neuronal NOS (Nos1) are expressed in the blood vessel lining and fibrous and cartilaginous tissues ${ }^{8-10}$. During these conversions the free radical NO is formed which stimulates bone cells to regulate bone remodelling by influencing osteoblasts and osteoclasts, and on the other hand influences the vascular reactivity ${ }^{11,12}$.

The second process within the arginine-citrulline-nitric oxide-metabolism that plays an important role during fracture healing is the conversion of arginine into ornithine by the arginase- 1 enzyme. Through the formation of polyamines, ornithine is a precursor for collagen synthesis, necessary for osteogenesis ${ }^{13}$. A schematic representation overviewing the arginine-citrulline-nitric oxide metabolism in shown in figure 7.1.

In addition to the previously shown effect, disturbances in the arginine substrate metabolism results in nonunion formation ${ }^{14}$, we hypothesized that stimulation of the arginine-citrulline-NO metabolism by oral supplementation of arginine or citrulline, as a precursor for arginine, will positively influence the fracture healing process in mice. 


\begin{tabular}{|cc|}
\hline VASCULAR REACTIVITY & BONE \& \\
$\&$ & CARTILAGE \\
INFLAMMATION & METABOLISM \\
\hline
\end{tabular}

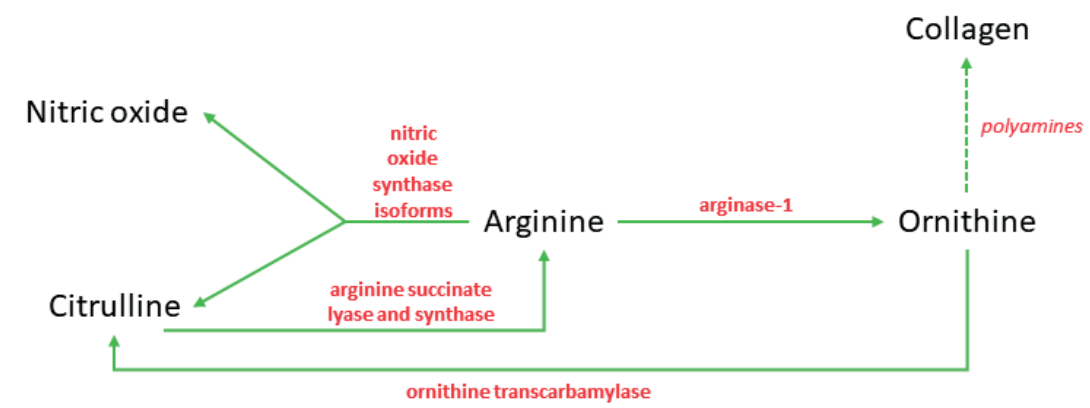

Fig. 7.1. Arginine-citrulline-NO metabolism. Schematic overview of the arginine-citrulline-NO metabolism with key amino acids and enzymes and its relation with inflammation and vascular reactivity versus bone and cartilage metabolism.

\section{MATERIALS AND METHODS}

\section{Animals and surgical procedure}

In this study, skeletally mature 20 to 24 week old female C57B16/J wild type mice were used. Before starting the experiments, all animals were allowed to acclimatize for at least two weeks during which they were socially housed (4-5 animals) in individually ventilated cages on a 12 hour day-night-cycle. During the entire experimental period, mice were fed water and standard chow ad libitum. The experimental protocols and surgical procedures were evaluated and approved by the local animal experimental ethics and well-fare committee (permit number DEC 2014-030).

Before surgery, anaesthesia was induced by placing the animals in a box flooded with isoflurane. Intraoperative analgaesia was administered by subcutaneous injection with $0.1 \mathrm{mg} / \mathrm{kg}$ buprenorphine. Mice were kept on a heating pad connected to a rectal probe to maintain adequate body temperature and prevent hypothermia during the surgical procedure. Inhalation anaesthesia during surgery was maintained on 1.5-2 \% isoflurane. Mice were placed in a prone position in an aseptic surgical field. The procedure was started with a lateral skin incision on the left femur, starting at the tail ranging towards the knee. By blunt dissection between the biceps and quadriceps femoris muscles, the femur was exposed. A 7 x $1.5 \times 0.5 \mathrm{~mm}$ plate was placed on the femur and fixed with four $2.0 \mathrm{~mm}$ angular stable screws after predrilling with a $0.33 \mathrm{~mm}$ drill bit. After fixation, a $0.45 \mathrm{~mm}$ osteotomy was performed using a Gigli wire saw and irrigation with $0.9 \%$ sodium 
chloride $(\mathrm{NaCl})$. All screws were untightened by half a turn to induce secondary fracture healing. The MouseFix ${ }^{\mathrm{TM}}$ screws, plates and instruments and the Gigli saw were obtained from RISystems, Davos, Switzerland. Skin and fascia were closed routinely with 5-0 vicryl (Ethicon, Bridgewater, NJ, USA).

The surgical procedure has previously been described by Gröngröft et $\mathrm{al}^{15}$. In the first two days after surgery, mice received $0.1 \mathrm{mg} / \mathrm{kg}$ s.c. buprenorphine every 10-14 hours as postoperative analgaesia. Animal well-being was monitored daily along with body weight registration. Mice were housed solitarily in individually ventilated cages for the purpose of adequate food intake measurements.

Daily supplementation of citrulline or arginine was performed by oral gavage. Citrulline was given in the concentration of $5 \mathrm{~g} / \mathrm{kg}$ bodyweight per day. The amount of arginine was $4.6 \mathrm{~g} / \mathrm{kg}$ bodyweight per day which in this concentration is isonitrogenous when compared with citrulline. These concentrations have been extrapolated from previous studies. The volume used for oral gavage was according to the general guidelines of $10 \mathrm{ml} / \mathrm{kg}$ body weight. Although citrulline has a slightly higher caloric value when compared to arginine, this difference was only $0.02 \%$ of the average general caloric intake of mice per day. Both amino acids were dissolved in $0.9 \% \mathrm{NaCl}$. Animals in the control group only received $0.9 \% \mathrm{NaCl}$.

All mice were randomly assigned to one of the three study groups. Each group consists of 15 animals, of which 6 were used for micro-computed tomography measurement, 6 for analysis of amino acid and enzyme concentrations and 3 for histologic assessment.

After 14 days, mice were euthanized under isoflurane inhalation anaesthesia using cardiac puncture to collect arterial blood samples. Blood was collected in prechilled heparanized cups (Sarstedt, Nümbrecht, Germany) on ice and centrifuged immediately ( $4^{\circ} \mathrm{C}, 15 \mathrm{~min}, 8,500 \mathrm{~g}$ ) to obtain plasma which was subsequently stored at $-80{ }^{\circ} \mathrm{C}$ for further analysis. Femurs were collected for micro-computed tomography by dislocation of the hip joint and storage at $4{ }^{\circ} \mathrm{C}$ until analysis. Femurs which were used for RNA and amino acid measurements were cleaned from all surrounding soft tissue and snap frozen in liquid nitrogen before storage at $-80^{\circ} \mathrm{C}$. In Figure 7.2 , an overview of the experimental setup is presented.

\section{Amino acid measurements}

To determine amino acid concentrations in femurs, the complete frozen femurs were crushed on liquid nitrogen using a pestle and mortar. Subsequently $30 \mathrm{mg}$ of tissue was placed in $2 \mathrm{ml}$ screw-cap vials with $0.1 \mathrm{~g}$ of glass beads ( $1 \mathrm{~mm}$ in diameter) and $250 \mu \mathrm{l}$ of $5 \%$ ice cold sulfosalicylic acid solution for deproteinization. Samples were then homogenized for 10 seconds thrice using the mini-bead beater (BioSpec Products, Bartlesville, Oklahoma, USA). After centrifugation (15 min, 4 ${ }^{\circ} \mathrm{C}, 50,000 \mathrm{~g}$ ) the obtained supernatant was diluted a 100 -fold in water and placed 
in the high-performance liquid chromatography apparatus. Amino acids were measured after pre-column derivatization using o-phthaldialdehyde (Thermo Scientific, Breda, The Netherlands). The resulting derivatives were separated in the chromatography column using a acetonitrile gradient and detected using fluorescence detector ${ }^{14,16}$.
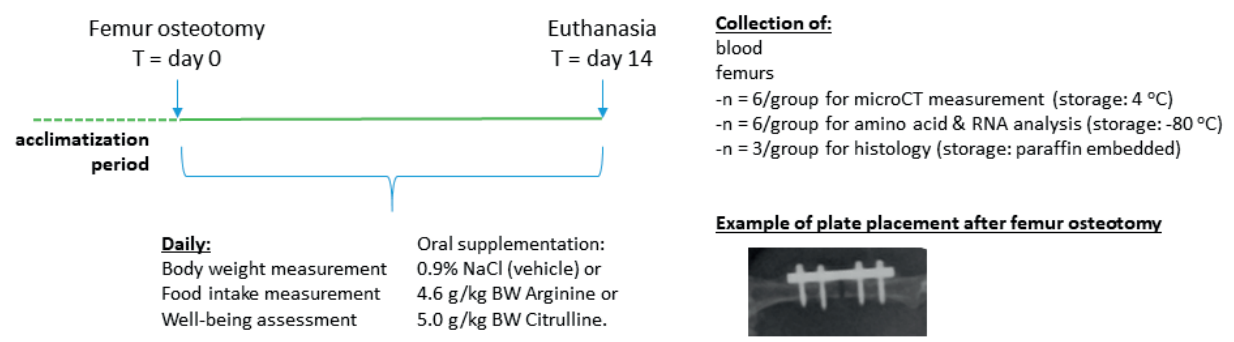

Fig. 7.2. Schematic representation of the experimental setup. After 2-week acclimatisation, a femur osteotomy was performed and, subsequently, mice were treated either as control or with arginine or citrulline supplementation daily for 2 weeks. Afterward, mice were euthanised using cardiac puncture and sample were collected.

\section{Micro-computed tomography}

Before the micro-computed tomography $(\mu \mathrm{CT})$ measurements, plates and screws were carefully removed from the femur with avoidance of any disruption of the newly formed callus.

Femurs were scanned by micro-computed tomography ( $\mu$ CT 40, Scanco Medical, Switzerland) at $70 \mathrm{kVp}$ and $114 \mu \mathrm{A}$ with $200 \mathrm{~ms}$ integration time. A Gaussian filter (sigma 0.8, support 1) was used for a partial suppression of the noise. Based on the described gray values, the degree of mineralization could be quantified. To ensure comparable measurements, bone volumes were measured between the first proximal and distal screw-holes next to the osteotomy. This region resembled 100 cross-sectional slices on the micro-CT-image.

\section{Radiologic assessment}

Post-mortem cross-sectional CT images of each femur were graded based on callus formation, rebridging of the cortex and remodelling of the callus by two blinded (bio)medically trained-investigators using the scoring scale previously described by Garrett et $a l^{17}$ (see table 7.1). 
TABLE 7.1 Radiographic scoring-scale based on callus formation, rebridgement of the cortices and remodelling of the callus.

\begin{tabular}{ll}
\hline score & definition \\
\hline 0 & No bridging, no callus formation \\
\hline 2 & No bridging, initiation of small amount of callus \\
\hline 3 & No bridging, obvious initial callus formation near fracture \\
\hline 5 & $\begin{array}{l}\text { No bridging, marked callus formation near and around fracture site } \\
\text { site }\end{array}$ \\
\hline 6 & $\begin{array}{l}\text { Rebridging of at least one of the cortices, marked and complete callus formation around } \\
\text { fracture site }\end{array}$ \\
\hline 7 & Rebridging of both cortices, and/or some resolution of the callus \\
\hline
\end{tabular}

\section{RNA analysis}

Crushed femur samples were incubated in TriSure (Bioline, London, UK) and homogenized using the mini bead-beater as mentioned before. Following homogenization, RNA was precipitated using isopropanol and centrifugation (30 min, $4{ }^{\circ} \mathrm{C}, 11,000 \mathrm{rpm}$ ). Obtained RNA pellets were washed with $80 \%$ ethanol and air-dried before dissolving in diethylpyrocarbonate-treated water (Sigma-Aldrich, Zwijndrecht, The Netherlands). To obtain adequate results, genomic DNA was removed using a DNase I treatment kit (Promega, Madison, WI, USA). Afterwards RNA was precipitated using $3 \mathrm{M}$ sodium acetate and RNA was dissolved in DEPCtreated water before cDNA synthesis was performed using the iScript cDNA synthesis kit (Biorad Products, Hercules, CA, USA). To perform quantitative polymerase chain reaction analysis, diluted cDNA was added to a SYBR green mix with the appropriate forward and reverse primers (see table 7.2 for genetic primer sequences). Cyclophilin A (Ppia) and $\beta$-actin (Actb) were used as house keeping genes. cDNA was amplified using the LC480 Lightcycler (Roche, Basel, Switzerland), with a 3-step program consisting of 40 cycles of denaturation (95 $\left.{ }^{\circ} \mathrm{C} 10 \mathrm{~s}\right)$, annealing $\left(60^{\circ} \mathrm{C}, 20 \mathrm{~s}\right)$ and elongation $\left(70{ }^{\circ} \mathrm{C}, 20 \mathrm{~s}\right)$. Expression of the different genes was analysed using the LC480 software. The geometric mean of the house keeping gene expressions was used as a normalization factor. Used primers were acquired from Sigma-Aldrich, Zwijndrecht, The Netherlands.

TABLE 7.2 Primer sequences for quantitative PCR

\begin{tabular}{|c|c|c|}
\hline Gene & Name & Primer sequence $\left(5^{\prime} \rightarrow 3^{\prime}\right)$ \\
\hline \multirow[t]{2}{*}{ Ppia } & Cyclophilin A Fw & TTCCTCCTTTCACAGAATTATTCCA \\
\hline & Cyclophilin A Rev & CCGCCAGTGCCATTATGG \\
\hline \multirow[t]{2}{*}{$A c t b$} & $\beta$-actin $\mathrm{FW}_{\mathrm{W}}$ & GACAGGATGCAGAAGGAGATTACTG \\
\hline & $\beta$-actin Rev & CCACCGATCCACACAGAGTACTT \\
\hline
\end{tabular}


TABLE 7.2 Continued

\begin{tabular}{|c|c|c|}
\hline Gene & Name & Primer sequence $\left(5^{\prime} \rightarrow 3^{\prime}\right)$ \\
\hline \multirow[t]{2}{*}{$\operatorname{Arg} 1$} & Arginase- $1 \mathrm{Fw}$ & GGAGAGCCTTCCTGCACTTT \\
\hline & Arginase-1 Rev & GTGCCTTGGTCTACATTGAACATAC \\
\hline \multirow[t]{2}{*}{ Nos 2} & iNOS Fw & TTGCAAGCTGATGGTCAAGATC \\
\hline & iNOS Rev & CAACCCGAGCTCCTGGAA \\
\hline \multirow[t]{2}{*}{ Nos3 } & eNOS Fw & TTAATGTGGCCGTGTTGCA \\
\hline & eNOS Rev & CTCTTGATGGAAGACAGGAGTTAGG \\
\hline \multirow[t]{2}{*}{ Bmp2 } & BMP2 Fw & GCTTCTTAGACGGACTGCGG \\
\hline & BMP2 Rev & GCAACACTAGAAGACAGCGGGT \\
\hline \multirow[t]{2}{*}{ Bmp7 } & BMP7 Fw & CCAAAGAACCAAGAGGCCC \\
\hline & BMP7 Rev & GCTGCTGTTTTCTGCCACACT \\
\hline \multirow[t]{2}{*}{ Vegfa } & VEGF FW & GCTTTACTGCTGTACCTCCACCA \\
\hline & VEGF Rev & GGGACTTCTGCTCTCCTTCTGTC \\
\hline \multirow[t]{2}{*}{ COL2A1 } & Collagen $2 \mathrm{FW}$ & GAGAGGTCTTCCTGGCAAAG \\
\hline & Collagen 2 Rev & AAGTCCCTGGAAGCCAGAT \\
\hline \multirow[t]{2}{*}{ ILG } & Interleukin $6 \mathrm{Fw}$ & GCTACCAAACTGGATATAATCAGGAAA \\
\hline & Interleukin 6 Rev & CTTGTTATCTTTTAAGTTGTTCTTCATGTACTC \\
\hline \multirow[t]{2}{*}{ NFKB1 } & $N F-\kappa B$ Fw & GCTACGGCGGCCTTCTG \\
\hline & NF- $\kappa$ B Rev & CAATCCGGTGGCGATCAT \\
\hline \multirow[t]{2}{*}{ IL1A } & Interleukin $1 \alpha \mathrm{FW}$ & AAAGAATCTATACCTGTCCTGTGTAATGAAA \\
\hline & Interleukin $1 \alpha$ Rev & GGTATTGCTTGGGATCCACACT \\
\hline \multirow[t]{2}{*}{ Cxcl2/Mip2 } & Mip2 FW & GCGCTGTCAATGCCTGAAGA \\
\hline & Mip2 Rev & TTTGACCGCCCTTGAGAGTG \\
\hline
\end{tabular}

FW: forward, Rev: reverse.

\section{Histological assessment of fracture repair}

After euthanasia, plates and screws were carefully removed from the femurs and samples were fixed in 4\% paraformaldehyde solution and decalcified using EDTA. Subsequently, femurs were embedded in paraffin and $4 \mu \mathrm{m}$ sections were prepared. For staining, sections were first deparaffinised in xylene and rehydrated from graded ethanol (100\% - 96\% - 70\%) to distilled water and afterwards stained for hematoxylin and eosin.

\section{Statistical analysis}

Statistical analysis of the obtained results was performed using GraphPad Prism 6 (GraphPad, San Diego, CA, USA). Normality of the results was checked using the Shapiro-Wilks test. All data presented in this paper are represented as means and standard error of the mean (SEM). ANOVA with post hoc Bonferroni correction was used to assess statistical significance. A Kruskal-Wallis test was performed on the discontinuous healing score measurements. In all cases, p-values below 0.05 were considered statistically significant. 


\section{RESULTS}

\section{Food intake and weight}

Food intake during the 14-day period was comparable between the control group and both experimental groups (4,5 g of regular chow per mouse per day, no further data shown). However, significant differences in animal total body weight were observed (Figure 7.3). During the initial post-operative days, animals in all groups lost about $1 \mathrm{~g}$ of weight ( $5 \%$ of total body weight). Over the complete 14day experimental period, control animals lost $0,1 \mathrm{~g}$ of their initial body weight. Arginine treated mice showed body weight which was comparable with their pre-operative weight. Citrulline treated mice showed an increase of $0.2 \mathrm{~g}$ over the 14-day period which was significant when compared to control animals ( $p$ $<0.01$ ). Additionally, both treated groups of mice showed a smaller decrease in body weight during the first two days postoperatively, when compared to control animals $(\mathrm{p}<0.05)$.

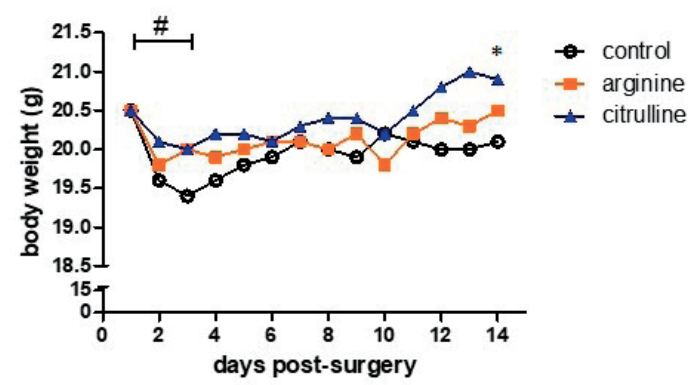

Fig. 7.3. Post-operative recovery. Average body weight per group per day. Mice in the control group are represented with the black line, arginine treated mice with orange and citrulline treated mice in blue. Standard deviations are not shown to increase readability of the figure. \# marks a significant difference, $\mathrm{p}<0.05$ in weight change within the first two days after the surgery between control mice and both treated groups. * marks a significant difference at day 14 between citrullinetreated mice vs. control animals.

\section{Callus formation}

Radiographic analysis showed that femurs in all three groups healed with a relatively low variations in formed callus between the individual mice in each group. Figure 7.4A shows representative three-dimensional rendered images of femurs after micro-CT analysis of the three experimental groups. According to the criteria mentioned in the Garrett scoring scale as presented in table 7.1, after 14 days of healing, control mice showed a marked callus formation near the fracture site without bridging of the cortices. Almost all arginine treated mice 
showed bridging of one of the cortices. In the citrulline treated group all mice showed bridging of the cortices with beginning resolution of the callus indicating a beginning remodelling phase. In addition, differences between citrulline treated mice and the control group reach statistical significance $(\mathrm{p}<0.05$, figure $7.4 \mathrm{~B})$. Figure $7.4 \mathrm{C}$ shows the bone volumes which were measured during the microCT analysis. These results were accordingly to the observed differences which were found using the radiographic scoring according to the criteria in table 7.1, with a statistical difference between samples obtained from the control group and the citrulline-treated animals $(\mathrm{p}=0.05)$. In figure $7.4 \mathrm{D}$, representative microscopic images are presented of histology slides after H\&E staining in femurs of each experimental group. Findings regarding callus formation are in line with observations found in micro-CT analysis.

a

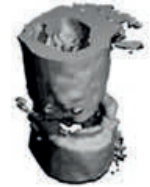

control

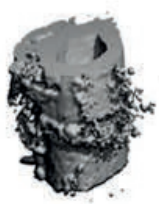

arginine

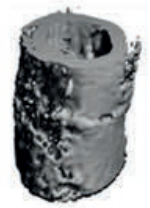

citrulline b

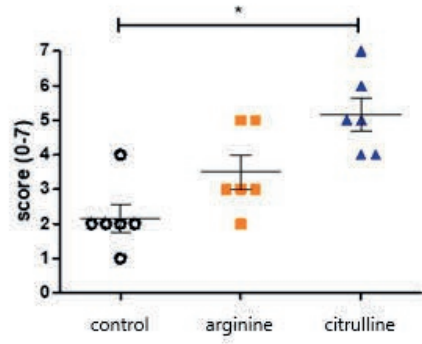

d

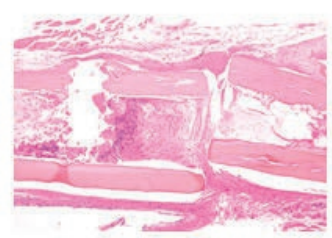

control

c

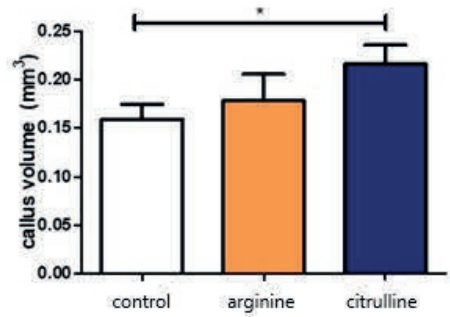

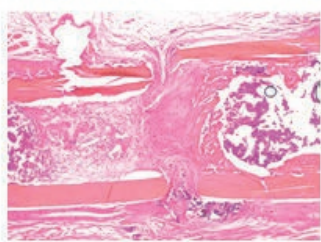

arginine

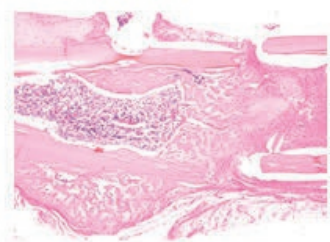

citrulline

Fig. 7.4. Callus formation. Panel A shows representative three-dimensional rendered images of callus formation in the three experimental groups from the osteotomy site towards the first proximal and distal screw-holes. Panel B: quantification of callus formation by assessment of the callus formation using the Garrett scoring scale (0-7). Panel C: quantification of bone volumes within the 
region represented in panel A. Legend: white represents the control group, orange represents arginine treatment, blue represents citrulline treatment. Levels op significance: *: $p<0.05$. Panel D shows representative images of histological staining of each treatment group using haematoxylin and eosin.

\section{Amino acid concentrations}

Arginine concentrations in femoral tissue of control animals was around $300 \mathrm{nmol}$ per gram of wet tissue. Treatment with arginine or citrulline did not result in significant higher arginine concentrations in femur tissue in these groups (Figure 7.5A). However, citrulline concentrations (Figure 7.5B) increased significantly in mice that received 14-day citrulline supplementation when compared to control mice that only received the vehicle $(\mathrm{p}<0.05)$. Between both treatment groups a favourable trend in citrulline concentrations was observed in citrulline treatment $(\mathrm{p}=0.053)$, compared to arginine treatment. The main influence of citrulline supplementation was observed in the obtained result of ornithine concentrations (Figure 7.5C). In citrulline treated animals, femur concentrations were significantly higher when compared to control animals $(\mathrm{p}<0.05)$ and also when compared to arginine treated mice $(\mathrm{p}<0.01)$.

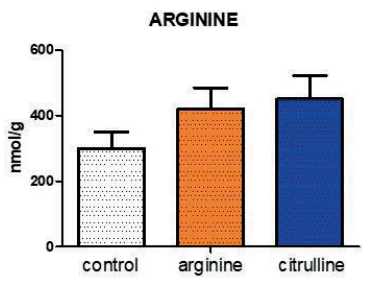

b

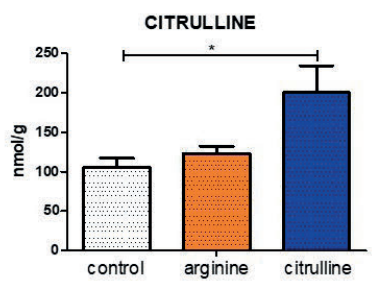

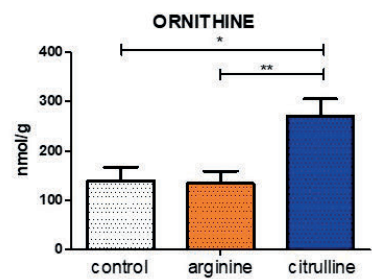

Fig. 7.5. Amino acid concentrations in femora. Arginine (panel A), citrulline (panel B) and ornithine (panel C) concentrations in femoral callus tissue in $\mathrm{nmol} / \mathrm{g}$ measured after euthanasia of the animals. White bars represent control animals which received $0.9 \% \mathrm{NaCl}$ supplementation, orange bars represent arginine treated mice, blue bars represent citrulline treated mice. Levels of significance: $*$ : $<<0.05, * *: p<0.01$

\section{qPCR}

Nos2 mRNA expression (Figure 7.6A) was significantly lower in femurs of mice treated with arginine $(\mathrm{p}<0.05)$ and citrulline $(\mathrm{p}<0.0001)$ when compared with control mice. Additionally, citrulline supplementation seemed to downregulate Nos2 expression stronger when compared with arginine supplementation ( $\mathrm{p}$ $<0.001$ ). Although Nos3 expression showed a similar trend (figure 7.6B), no significant differences were found between the three groups. Also for Arg-1 
expression, no significant differences were found in the three groups $(p=0.21$ between arginine and citrulline treated groups, figure 7.6C).

To assess the influence of bone morphogenetic proteins, mRNA expression of $B m p 2$ and Bmp7 was measured. No significant differences were present in either of the groups in both genes (respectively figure 7.6D and 7.6E).

Angiogenesis was assessed using mRNA expression levels of vascular endothelial growth factor (VEGF, figure 7.6F). Vegfa expression showed a significant 2-fold increase in citrulline treated animals in comparison with control mice $(\mathrm{p}<0.01)$. In addition, expression in citrulline treated animals was also higher when compared with arginine treated animals $(\mathrm{p}<0.05)$.

Where arginase-1 (a precursor for collagen synthesis) showed no differences between the groups, a difference was however observed in collagen type IIexpression. Expression in citrulline treated mice was higher when compared with both the control group and with mice that received daily arginine supplementation (both $\mathrm{p}<0.05$, figure $7.6 \mathrm{G}$ ).

To further investigate the role of inflammation, Il-6, NF-kB, Il-1 and Cxcl2/Mip2 mRNA expression was measured (figures 7.6H, 7.6I, 7.6J and 7.6K respectively). Only $\mathrm{Cxcl} 2 / \mathrm{Mip} 2$ showed significant results with lower expression in arginine treated animals $(\mathrm{p}<0.01)$ and citrulline treated animals $(\mathrm{p}<0.05)$ when compared to the control group. 

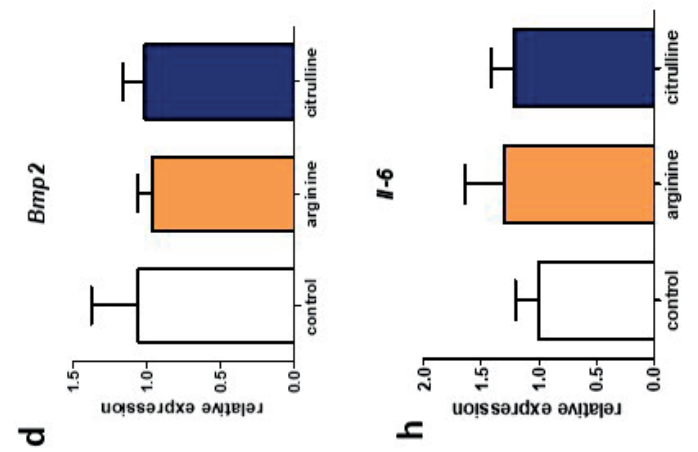

品

oi

$\Xi 0$

记 $v$

एँ

$\frac{0}{-1}$

on

$\exists$ V

产

8

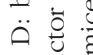

;

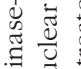
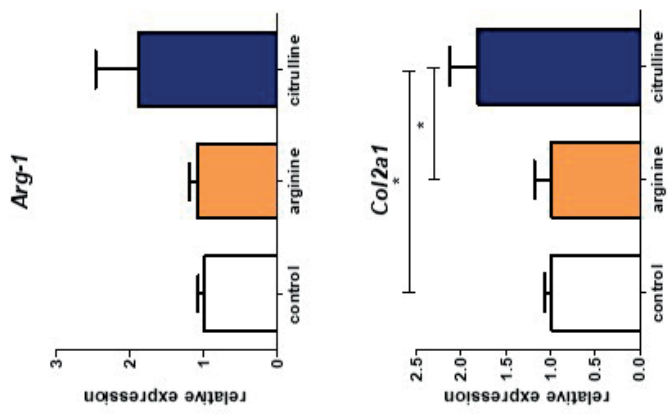

wo
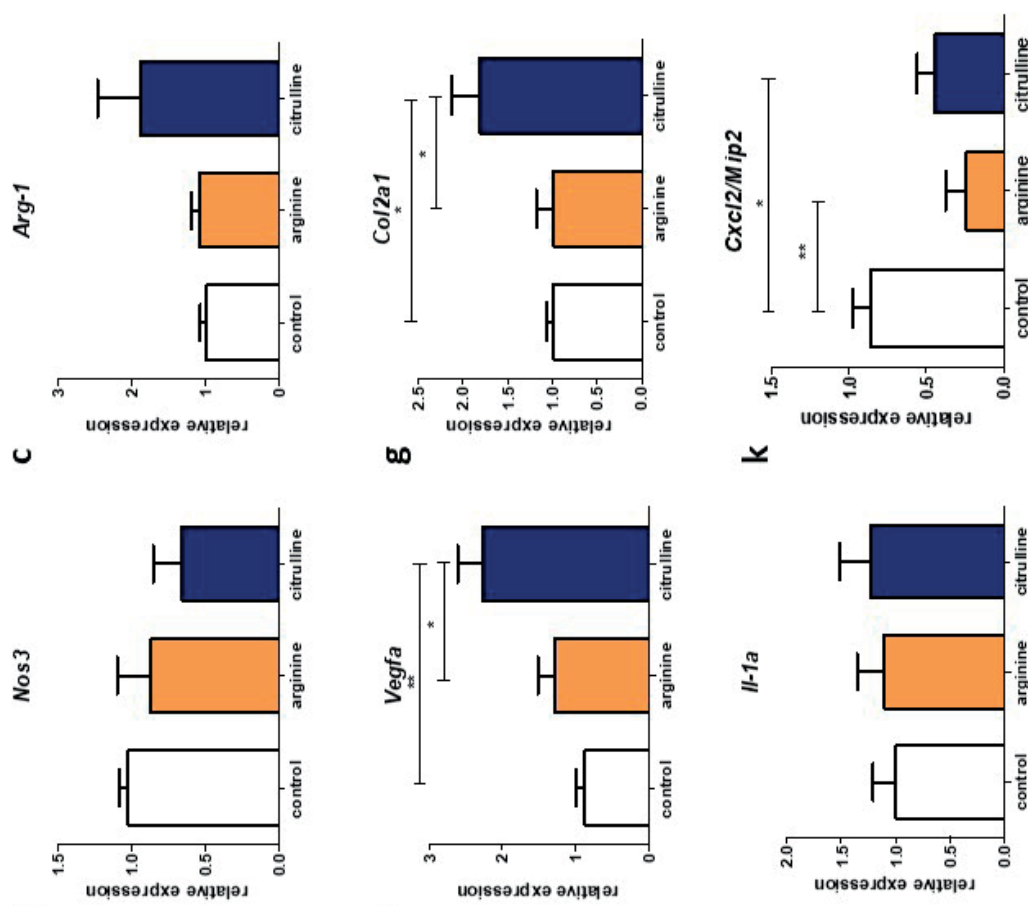

요

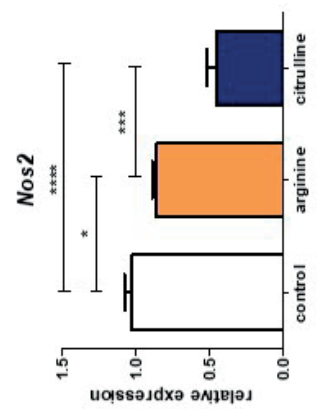

4
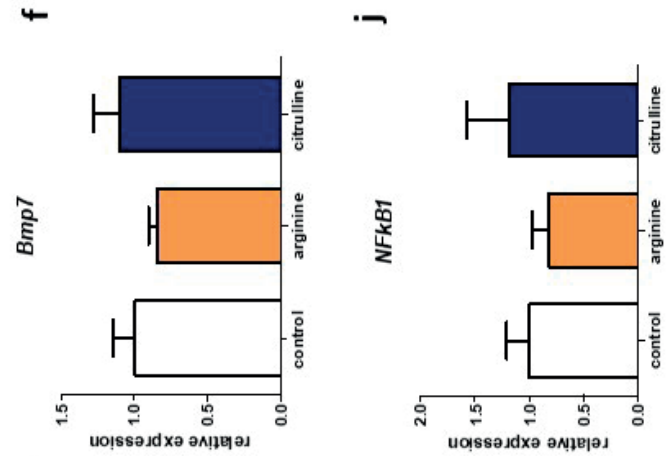

$\boldsymbol{\omega}$

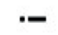

च

항

茟范荡

进

च

동

章 


\section{DISCUSSION}

In the present study we show that daily oral supplementation of citrulline in mice that underwent a controlled femur osteotomy increases new bone formation when compared with untreated mice. The effect supplementation of arginine did not influence bone formation. Next to bone citrulline concentrations, also ornithine concentrations in the femurs from citrulline-treated mice are increased which may indicate the progress towards collagen synthesis through polyamine production. The increased ornithine concentrations are accompanied by enhanced expression of arginase 1 and significant increased collagen II expression in these mice. Nitric oxide synthase enzymes show lower expression profiles on RNA level in citrulline treated mice when compared to control mice. Increased expression of vascular endothelial growth factor provides insight into the significant improved angiogenesis after citrulline supplementation. Furthermore, mice treated with either of the amino acids showed an increased weight gain during the 14-day study period suggesting an improved post-operative recovery.

Generally, absence of interfragmentary motion between two fracture parts in mice occurs after three weeks of bone healing ${ }^{18}$, suggesting adequate callus formation and cortical bridging of the fracture gap. Subsequently, the process of callus resolution and remodelling will continue. In the present study we hypothesize that stimulation of the arginine-NO-citrulline metabolism will enhance fracture healing, thus shortening the period until interfragmentary motion is absent. Therefore, a healing period of 14 days was chosen to assess the outcome of metabolic stimulation in these mice.

Although several studies already investigated the influence of a pharmacologic 8,19 or genetic ${ }^{14}$ disturbance in the arginine-citrulline-nitric oxide metabolism on the bone healing process, we here report for the first time the results of stimulation of bone healing in a reliable, representative and reproducible murine bone healing model.

In patients, it is known that availability of arginine and related amino acids are necessary to achieve adequate fracture healing ${ }^{20}$. Additionally, it is known that during prolonged hospitalisation after hip fracture surgery, mainly elderly people suffer from a substantial loss of skeletal muscle mass ${ }^{21}$. Therefore, nutritional supplementation with amino acids is preferable perioperatively and during rehabilitation ${ }^{22}$. Additionally, mainly citrulline supplementation is known to increase skeletal muscle and total body weight in both in vivo animal experiments ${ }^{23}$ as well as in humans ${ }^{24}$. The significant body weight gain and mainly the reduced weight loss during the first days as observed in mice that were treated with citrulline may point towards this enhanced post-operative recovery and is in line with previous animal research investigating the reversal of detrimental effects 
after protein malnutrition in bone healing ${ }^{25}$.

Amino acid measurements in femoral tissue show both increased citrulline concentrations as well as ornithine concentrations after supplementation with citrulline when compared with tissues obtained from control mice. Conversion of arginine into ornithine is mediated by arginase-1. Our results show no significantly higher expression of arginase-1, however, through formation of polyamines, ornithine is a precursor for collagen synthesis, which was found to induce an increased expression on mRNA level in our femur samples. Under normal healing conditions, collagen II expression peaks after 14 days of bone healing ${ }^{26}$ and gradually declines thereafter. One limitation regarding the current study is the lack of multiple time points at which the influence of stimulation with either of the amino acids is measured. Future studies should therefore focus on one or more time points ranging further in the healing process to assess the possible differences at later stages during the fracture healing process. Therefore, it is expected that collagen II expression would be higher when measured after 7 days of fracture healing in our current experimental setup.

Conversion of arginine into citrulline is mediated by the nitric oxide synthase enzymes. Mainly Nos 2 and Nos3 are of influence during the initial healing responses, the neuronal Nos1 is only expressed during the later remodelling phase of fracture healing 9 , thus having no influences on the processes studied in our current investigation.

Nos2 expression was significantly lower in femurs obtained from mice after citrulline treatment, indicating a beneficial and more advanced systemic inflammatory response after 14 days in these mice, compared with prolonged inflammation in the other experimental groups, which additionally is underlined by the results found in $\mathrm{Cxcl} 2 / \mathrm{Mip} 2 \mathrm{mRNA}$ expression.

An adequate inflammatory response and fracture hematoma formation is necessary during the fracture healing process with recruitment and activation of macrophages and subsequent neutrophils and production of pro-inflammatory cytokines, chemokines and growth factors, as it is known that disturbances is these inflammatory signalling processes often lead to development of delayed union and nonunion later in the healing process. From our previous research it is known that deletion of the Nos 2 gene results in prolonged inflammation and higher degrees of neutrophil influx in and around the fracture callus ${ }^{14}$ and subsequently resulting in delayed union.

Nos3, mainly expressed during in the endothelium during the vascular ingrowth of capillaries in and around the callus ${ }^{10}$, showed no significant differences between the studied groups. However, vascular endothelial growth factor ( Vegfa) was significantly increased in citrulline treated mice that showed increased bone formation, indicating a activation of angiogenesis while new vessels have not 
been formed yet, thus Nos3 is not yet expressed.

In the current model, a controlled mid shaft femur osteotomy is induced. However, patients who sustain a fracture usually show different fracture patters due to the mechanics related to the trauma. Another future perspective is to investigate if stimulation of fracture healing by arginine-citrulline-nitric oxide metabolism in a model which resembles these trauma mechanics and fracture patterns more closely. Different small animal models with closed fracture induction methods are available ${ }^{27,28}$. Additionally, a translation into the human clinical setting is obviously the ultimate goal of the performed research. Although bone healing metabolism is comparable between mice and humans, small differences remain present next to differences in biomechanical loading, i.e. lack of a Haversian system in rodents and different kinetics in metabolic processes.

In conclusion, we showed that daily oral citrulline supplementation beneficially affects the bone healing response after a controlled femur osteotomy in mice. 


\section{REFERENCES}

1. van Staa TP, Dennison EM, Leufkens HG, Cooper C. Epidemiology of fractures in England and Wales. Bone 2001; 29(6): 517-22.

2. Mills LA, Aitken SA, Simpson A. The risk of non-union per fracture: current myths and revised figures from a population of over 4 million adults. Acta orthopaedica 2017: 1-6.

3. Bastian OW, Kuijer A, Koenderman L, et al. Impaired bone healing in multitrauma patients is associated with altered leukocyte kinetics after major trauma. J Inflamm Res 2016; 9: 69-78.

4. Court-Brown CM, McQueen MM. Nonunions of the proximal humerus: their prevalence and functional outcome. The Journal of trauma 2008; 64(6): 1517-21.

5. Bishop JA, Palanca AA, Bellino MJ, Lowenberg DW. Assessment of compromised fracture healing. J Am Acad Orthop Surg 2012; 20(5): 273-82.

6. Calori GM, Albisetti W, Agus A, Iori S, Tagliabue L. Risk factors contributing to fracture nonunions. Injury 2007; 38 Suppl 2: S11-8.

7. Meesters DM, Wijnands KAP, Brink PRG, Poeze M. Malnutrition and Fracture Healing: Are Specific Deficiencies in Amino Acids Important in Nonunion Development? Nutrients 2018; 10(11).

8. Diwan AD, Wang MX, Jang D, Zhu W, Murrell GA. Nitric oxide modulates fracture healing. J Bone Miner Res 2000; 15(2): 342-51.

9. Zhu W, Diwan AD, Lin JH, Murrell GA. Nitric oxide synthase isoforms during fracture healing. $J$ Bone Miner Res 2001; 16(3): 535-40.

10. Zhu W, Murrell GA, Lin J, Gardiner EM, Diwan AD. Localization of nitric oxide synthases during fracture healing. J Bone Miner Res 2002; 17(8): 1470-7.

11. Chae HJ, Park RK, Chung HT, et al. Nitric oxide is a regulator of bone remodelling. J Pharm Pharmacol 1997; 49(9): 897-902.

12. Corbett SA, McCarthy ID, Batten J, Hukkanen M, Polak JM, Hughes SP. Nitric oxide mediated vasoreactivity during fracture repair. Clin Orthop Relat Res 1999; (365): 247-53.

13. Vittur F, Lunazzi G, Moro L, et al. A possible role for polyamines in cartilage in the mechanism of calcification. Biochim Biophys Acta 1986; 881(1): 38-45.

14. Meesters DM, Neubert S, Wijnands KA, et al. Deficiency of inducible and endothelial nitric oxide synthase results in diminished bone formation and delayed union and nonunion development. Bone 2016; 83: 111-8.

15. Grongroft I, Wissing S, Meesters DM, et al. Development of a novel murine delayed secondary fracture healing in vivo model using periosteal cauterization. Arch Orthop Trauma Surg 2019.

16. van Eijk HM, Rooyakkers DR, Deutz NE. Rapid routine determination of amino acids in plasma by high-performance liquid chromatography with a 2-3 microns Spherisorb ODS II column. $J$ Chromatogr 1993; 620(1): 143-8.

17. Garrett IR, Gutierrez GE, Rossini G, et al. Locally delivered lovastatin nanoparticles enhance fracture healing in rats. Journal of orthopaedic research : official publication of the Orthopaedic Research Society 2007; 25(10): 1351-7.

18. Garcia P, Histing T, Holstein JH, et al. Rodent animal models of delayed bone healing and non- 
union formation: a comprehensive review. European cells $\&$ materials 2013; 26: 1-12; discussion -4 .

19. Turner CH, Takano Y, Owan I, Murrell GA. Nitric oxide inhibitor L-NAME suppresses mechanically induced bone formation in rats. Am J Physiol 1996; 270(4 Pt 1): E634-9.

20. Wijnands KA, Brink PR, Weijers PH, Dejong CH, Poeze M. Impaired fracture healing associated with amino acid disturbances. Am J Clin Nutr 2012; 95(5): 1270-7.

21. Kouw IWK, Groen BBL, Smeets JSJ, et al. One Week of Hospitalization Following Elective Hip Surgery Induces Substantial Muscle Atrophy in Older Patients. J Am Med Dir Assoc 2019; 20(1): $35-42$.

22. Kramer IF, Blokhuis TJ, Verdijk LB, van Loon LJC, Poeze M. Perioperative nutritional supplementation and skeletal muscle mass in older hip-fracture patients. Nutr Rev 2019; 77(4): 254-66.

23. Villareal MO, Matsukawa T, Isoda H. L-Citrulline Supplementation-Increased Skeletal Muscle PGC-1alpha Expression is Associated With Exercise Performance and Increased Skeletal Muscle Weight. Mol Nutr Food Res 2018: e1701043.

24. Martone AM, Lattanzio F, Abbatecola AM, et al. Treating sarcopenia in older and oldest old. Curr Pharm Des 2015; 21(13): 1715-22.

25. Day SM, DeHeer DH. Reversal of the detrimental effects of chronic protein malnutrition on long bone fracture healing. J Orthop Trauma 2001; 15(1): 47-53.

26. Grongroft I, Heil P, Matthys R, et al. Fixation compliance in a mouse osteotomy model induces two different processes of bone healing but does not lead to delayed union. J Biomech 2009; 42(13): 2089-96.

27. Histing T, Garcia P, Holstein JH, et al. Small animal bone healing models: standards, tips, and pitfalls results of a consensus meeting. Bone 2011; 49(4): 591-9.

28. Holstein JH, Matthys R, Histing T, et al. Development of a stable closed femoral fracture model in mice. The Journal of surgical research 2009; 153(1): 71-5. 



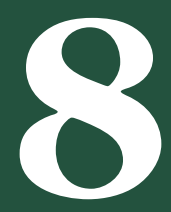

General discussion 


\section{General discussion}

The research presented in this thesis investigated the influence of the argininecitrulline-nitric oxide-metabolism during the healing of fractured bones and in occurrence with complications during the healing process, such as nonunion development.

Currently, approximately 1 in 100 persons within the general population per year will sustain a fracture ${ }^{1}$, with increasing additional risks of osteoporotic fractures ranging between $13-50 \%{ }^{2,3}$. The observed lifestyle changes in mainly the older population, with people being more active until a higher age result in a higher number of domestic-, traffic- and sports-related accidents and fractures ${ }^{4}$.

Aspects influencing the fracture healing process can be divided into fracturedependent and patient-dependent factors ${ }^{5,6}$. As fracture-dependent factors, characteristics as the fractured bone, fracture type (open or closed fracture, with/ without soft tissue loss) ${ }^{7}$ and associated infectious complications, fracture pattern (with/without bone loss possibly resulting in critically sized defects) ${ }^{8}$ and fracture localization? can be identified. The patient-dependent factors can be subdivided into two groups. The first group of patient-dependent factors are mainly influenced by comorbidities, such as diabetes mellitus ${ }^{10,11}$, metabolic or genetic disorders or cancer $^{12}$ resulting in a poor vascular state and disturbed metabolism or by treatments for these comorbidities (e.g. use of non-steroidal anti-inflammatory drugs, radiotherapy). The second group of patient-dependent factors are life-style factors, such as alcohol ${ }^{13}$, nicotine use ${ }^{14,15}$, and the patient's nutritional status ${ }^{16-18}$. This thesis mainly focusses on aspects of malnutrition as factor in development of nonunion.

Malnutrition is defined as "a state resulting from lack of intake or uptake of nutrition that leads to altered body composition and body cell mass leading to diminished physical and mental function and impaired clinical outcome from disease" $^{19}$. For proteins specifically, the recommended daily allowance for adults is $0.8 \mathrm{~g} / \mathrm{kg}$ body weight per day ${ }^{20}$, with advises for elderly active adults $(>65$ year), with possible comorbidities up to $>1.5 \mathrm{~g} / \mathrm{kg}$ body weight per day to be able to maintain lean body mass and function ${ }^{21}$. The tendency for malnourishment within the elderly age group makes them at risk for complicated fracture healing due to disturbances within normal physiologic bio-molecular processes ${ }^{22}$ and influences fracture healing via three ways. First, (mainly protein and amino acid) malnourishment directly leads to diminished bone formation resulting in increased postoperative complications such as delayed union and nonunion development in these patients ${ }^{16,18,23}$. Secondly, malnourished patients have a higher fall-risk due to associated muscle weakness both at home and in hospital ${ }^{20,24}$, contributing to the absolute number of fractures and subsequent prevalence of fracture healing complications. Finally, independent of the underlying illness, malnourishment 
leads to prolonged hospital recovery due to muscle wasting and reduced muscle function, even after uncomplicated surgery ${ }^{25}$, with increased mortality ${ }^{26,27}$, more post-operative complications (infections) and an increased number of re$\operatorname{admissions}^{28}$.

Supplementation with (non/semi)-essential amino acids are widely used and investigated to improve malnourishment in (hospitalized) patients with different diseases such as cardiac disease, sepsis, cancer and liver ${ }^{29-31}$. Along with an improved nutritional status contributing to recovery, amino acids are also known to have beneficial effects on the development of post-surgical infections and wound healing ${ }^{32}$, as discussed in detail below.

\section{Arginine-citrulline-nitric oxide metabolism}

A cellular signalling molecule of particular interest and importance during fracture healing is nitric oxide (NO). NO is a free radical which is solely produced during the conversion of the semi-essential amino acid arginine into citrulline by one of the isoforms of the nitric oxide synthase enzyme. Nitric oxide synthase isoform 1 (Nos1) in mainly found in neuronal cells and functions as a neurotransmitter. Both Nos1 and Nos3 (which acts in the endothelium) are calcium-dependent and expressed constitutively. Nos2 is involved during the immune response and is induced during inflammatory conditions. Via conversion of arginine into ornithine by the arginase enzyme, arginine also acts as a precursor for subsequent formation of polyamines and proline, necessary for collagen synthesis during bone formation. Nos3 mainly plays a role during angiogenesis and the capillary in-growth in the fracture callus.

Through the above mentioned metabolism, nitric oxide is able to regulate bone remodelling ${ }^{33,34}$, stimulate bone cells ${ }^{35}$ and influence vascular reactivity ${ }^{36}$, which all three are important processes during the fracture healing cascade of inflammation, callus formation and remodelling ${ }^{37,38}$.

\section{Animal model}

To adequately assess the involvement of the arginine-citrulline-NO metabolism during fracture healing, it is necessary to use a reliable and reproducible animal model to investigate the ongoing metabolic and (patho)physiologic processes and responses following treatment interventions. Multiple reviews ${ }^{39-41}$ have been published summarizing and comparing the different in vivo models which can be used to study normal fracture repair, delayed union development and nonunion development. Most models have been created to assess the biomechanical and biomolecular influences during bone healing. The different fracture repair models can either be classified as closed models ${ }^{42,43}$, with a better representation of the clinical situation together with containment of the closed fracture hematoma, 
or as open models in which a fracture or osteotomy is induced surgically and fixed with plate-screw osteosynthesis, intramedullary pins or external fixators and which are more reproducible and standardized ${ }^{42,44-50}$.

The development of our in vivo murine femur osteotomy model with open reduction and internal plate-screw fixation provides an optimal continuum and intermediary between delayed union and either normal bone healing or nonunion development by additional usage of periosteal cauterization. Additionally, it can be used to investigate improvement or disturbance of bone healing processes resulting from pharmacologic, metabolic or genetic interventions ${ }^{51}$.

A key factor in this model is the addition of periosteal injury by cauterization after a femoral osteotomy with plate-screw osteosynthesis, which prolongs the healing period by 7 to 14 days and shows decreased volumes of woven bone formation in and around the osteotomy site. Damage to the periosteum leads to an impaired blood supply $y^{52-55}$ and results in lower release and proliferation of osteoprogenitors and to a reduced capacity to form cartilage and bone without migration of chondrocytes or osteoblastic cells towards the osteotomy site ${ }^{56,57}$. The observed delay is considered to be clinically relevant since normalized by averaged healing time in mice ( 4 weeks) ${ }^{58}$ and humans (16 to 20 weeks), it can be extrapolated that the observed delay of about 1-2 weeks in our mice model would correspond to delayed healing in humans by around 4-6 weeks.

Mice lack an exact copy of the human system of Haversian canals which might be considered as a limitation although a comparable mechanism using resorption cavities is present ${ }^{59,60}$. A main advantage of mice is the benefit of being easily genetically modifiable which enables deletion of selected genes coding for specific enzymes that might influence the metabolism of interest ${ }^{51}$.

\section{Deletion of nitric oxide synthase enzymes}

Our first study describes the influence of absence of the endothelial and inducible nitric oxide synthases on bone healing in this model ${ }^{51}$ of delayed union development.

Deletion of either of these genes in mice resulted in transition of delayed union into nonunion of the femur osteotomy ${ }^{61}$. Additionally, prolonged inflammation was observed in these femurs as shown by increased neutrophil influx in and around the callus area during the later stages of fracture healing, thus indicating the important role of NO to regulate the balance of hypo- and hyperinflammation during the healing process. The volumes of newly formed bone in the callus region were lower in both knockout groups when compared with regular wild type animals, however, Nos 2 deficient animals showed hampered periosteal callus formation, whereas in Nos 3 deficient mice the endosteal callus volume was lower, suggesting different pathways or mechanisms in which the fracture healing is disturbed in these animals. These results are in line with previous studies in which 
the different nitric oxide synthase isoforms were shown to have a temporal ${ }^{62}$ and spatial $^{63}$ expression during bone healing when compared to unfractured femoral cortices $^{64}$. Here, Nos 2 was present at the edge of the periosteal callus and mainly during the initial inflammatory stage of fracture healing, which corresponds with findings after inflammatory stimulation of bone marrow macrophages and osteoblast during in vitro culturing ${ }^{65}$. Nos3 was expressed mainly in the lining of blood vessels of in-grown capillaries in the callus during the secondary bone formation. Similar trends were also found when assessing NOS expression on protein levels ${ }^{35,36}$. Additionally, Nos 2 knockouts exhibit lower torsional strength when compared to wild type mice ${ }^{66}$ during strength and stiffness testing. Further emphasis on the importance of bone healing is placed by studies which investigated suppression of NO syntheses by feeding rodents with L-nitroso-arginine methyl ester (L-NAME), a non-selective NOS inhibitor, further emphasized the importance of NOS, as this resulted in smaller callus cross-section areas and lower maximum failure load during biomechanical testing of rat femurs ${ }^{64}$. Finally, as observed, the arginine availability in Nos 2 and Nos 3 knockout mice was significantly decreased at 28 days of fracture healing indicating the importance of NOS presence and arginine availability on fracture healing to achieve an adequate repair by both improving the inflammatory as well as the metabolic response.

\section{Human studies}

Essential and non-essential amino acid concentrations are both known to be decreased in plasma of geriatric trauma patients when compared to healthy controls, indicating the aforementioned influence of malnutrition during fracture healing. Diminished ornithine plasma concentrations in patients hospitalized after a major trauma indicate the necessity of amino acids in active anabolic reactions ${ }^{17}$. Previous research in our group already found significant changes in amino acid concentrations in patients with long bone nonunions ${ }^{67}$. Atrophic nonunions had lower concentrations of all amino acids related to the arginine-citrulline-NO metabolism, where in hypertrophic nonunion samples, elevated concentrations of arginine were present, while concentrations of ornithine were also lowered.

Autologous bone and bone marrow grafting using the reamer-irrigator-aspirator (RIA) is one of the regular treatment options for long bone nonunions and which adheres to the Pentagon concept ${ }^{68}$ in bone healing. Effectiveness of autologous bone grafting in restoration of a nonunion can vary considerably between patients from 80 to $90 \%{ }^{68,69}$. A combination of clinical and radiological factors might be used to predict outcome after treatment ${ }^{70-72}$, however, knowledge is limited.

One might think that the high incidence of fractures might result in high numbers of possible patients which can be included into fracture healing and nonunion development studies. However, the large heterogeneity of the 
different characteristics in patients which might have additional influence on the healing process (i.e. fracture type and localization ${ }^{71,73}$ ) and influences on the arginine-citrulline-nitric oxide metabolism (wide age-ranges and according comorbidities ${ }^{7-77}$ ) will result in a relative low number of patients which can be directly compared in an ideal situation.

When outcome of RIA treatment success was assessed, patients were classified either into successful (consolidation of the nonunion) or unsuccessful (persisting nonunion) treatment. In bone tissue which was obtained from healthy bone distant from the nonunion site, significantly increased Nos2 expression was found in tissue obtained from patients with persisting nonunions after RIA treatment. This suggests the presence of a whole-body prolonged inflammatory response resulting in production of several proinflammatory cytokines such as interleukin-1 (IL-1), tumor necrosis factor- $\alpha$ (TNF- $\alpha$ ) and interferon- $\gamma$ (IFN- $\gamma$ ). This coincides with lower arginine concentrations in reamed-intramedullary-aspirate in these patients which indicates an increased catabolic response in this patient group $^{78}$. Additionally, the lower expression of arginase-1 and lower ornithine concentrations in patients with persisting nonunions reflects an disturbed anabolic response in the bones of these patients with decreased formation of collagens via polyamines ${ }^{79}$. Both previous observations combined may be the cause of the underlying inadequate response to the RIA treatment ${ }^{38,80}$, and serve as evidence that the whole-body metabolic response (distant from the nonunion site) is important during bone healing treatment of nonunions.

In our patient cohort, the nonunion scoring scale (NUSS) was found to be a predictor of the success rate of the RIA treatment for the nonunion. Captivatingly, the activity of the inflammatory response as measured by Nos 2 expression in the grafted material was an even better predictor of RIA therapy success, and may serve as biomarker of this prolonged inflammatory response.

\section{Improvement of bone healing}

Although it has already been established that disturbance of the arginine-citrullinenitric oxide metabolism, genetically or pharmacologically induced, during bone healing will result in inadequate fracture repair in animal model ${ }^{61,64,66}$ and with abnormal amino acid availability in patients with nonunions ${ }^{17,67}$, evidence of reversal of inadequate bone healing or stimulation of fracture repair is scarce and lacking fundamental mechanistic background ${ }^{81}$.

Our results indicate that mainly citrulline supplementation stimulates callus formation via the arginine-NO pathway in our mouse model, as seen by increased expression of collagen which is produced via ornithine, proline and polyamine precursors ${ }^{79}$. Additionally, the inflammatory response after the trauma was improved by lower Nos 2 and Cxcl2/Mip2 expression at 14 days of healing 
in citrulline-treated mice when compared with control animals. In comparison, mice in which a nonunion is present ${ }^{61}$, a hyperinflammatory state is present with prolonged inflammation and expression (MPO) after six weeks of fracture repair. Comparable hyperinflammatory processes are also found in patients who present symptoms of delayed/absent bone repair. Lastly, mice that received amino acid treatment showed a better post-operative recovery indicated by significantly increased body weight after 14 days and a lower initial decrease in body weight during the first two post-operative days although food-intake between the groups was comparable and the additional caloric intake was negligible.

Where Nos2 expression improved after citrulline stimulation, Nos3 expression did not change. However, vascular endothelial growth factor-a (Vegfa) was drastically increased, indicating a activation of angiogenesis, while new vessels have not been formed yet, thus Nos3 could not yet be expressed. Interestingly, where during bone healing stimulation Nos2 and Nos3 showed a comparable trend, Vegfa expression showed an opposite effect. During nonunion formation however, Nos 2 and Vegfa were comparable with decreased expression, while Nos3 showed a significant increase. Citrulline stimulation was shown to reverse the opposing effect of Nos 2 and $\mathrm{Veg} f a$

The consolidation of the osteotomy in mice treated with citrulline supplementation after 14 days indicates a shortened healing period of approximately 1 week. Translated into the clinical situation, on extrapolation this might lead to a drastically shortened healing period of at least on month. To be able to adequately investigate the influence of citrulline supplementation in the clinical setting, a pilot study in patients with bone healing difficulties resulting in segmental defects should be conducted and compared to a control group.

\section{Future perspectives}

The results obtained in our animal studies as well as those that were found in human samples strongly suggest a significant impact of the arginine-citrullinenitric oxide metabolism during fracture repair and nonunion development.

Up until now, a femur osteotomy has been used to link the metabolic changes which occur to the formation of newly formed bone tissue. Inducing an osteotomy is the most ideal model in terms of reliability and reproducibility to investigate the physiologic and metabolic responses of bone healing. However, in the clinical setting, fractures have an almost unlimited range of different patterns due to the different trauma mechanisms. Furthermore, where the osteotomy model is an open surgical procedure, most fractures are closed. Different small animal closed fracture models that resemble the clinical situation are available and use dropping weights, three point bending and other mechanistics to induce a fracture in a relatively controlled setting ${ }^{40,48}$, although a closed fracture has a low 
risk for nonunion development in the clinical setting. Moreover, where in our supplementation study the degree of callus formation was investigated at one time point, the introduction of titanium covered PEEK (polyether-ether-ketone) plates $^{82}$ in murine and rodent research enables one to follow the callus formation in animals at several time points over a longer period, adding to refinement and reduction with the three R's adagio in animal research.

Another interesting point to study is the importance of periosteal injury during fracture healing as it was shown to be of importance in femur osteotomies either healing normally or progressing towards delayed union or nonunion ${ }^{51,61}$. The periosteum contains fibroblasts and progenitor cells which develop into osteoblasts and chondroblasts which are essential during bone healing after sustaining a fracture. In cases with a high degree of periosteal layer damage or loss, increased fracture healing complications are observed. All underlying mechanisms are not clear yet, however, our samples showed correlations between the inducible nitric oxide synthase and Nos3 and Vegfa and which showed different results during disrupted bone healing on one hand and normal physiological repair process otherwise. An interrupted (periosteal) blood flow to the fracture site is already known to decrease callus formation ${ }^{57}$, and the stability of the fracture drastically influences the angiogenic response during the bone healing period ${ }^{83}$. Additionally, results of a pilot experiment show both an increased Vegfa and Nos 2 upregulation when chondrocytes are stimulated in vitro using both bone morphogenetic protein 2 as well as a selective Nos2 inhibitor (1400W) as supplement for 10 days. Strikingly, when fracture healing was inhibited in our Nos deficient mice, Nos2 and Vegfa showed similar trends in genetic expression, however, when fracture healing was stimulated using amino acid supplementation in regular wild type mice, Nos 2 and Vegfa showed opposing expression results. The coupling between both markers is a worthwhile item for future investigations during the bone healing response.

With citrulline's known beneficial effects on the immune response during sepsis or infection ${ }^{31,84}$, it would also be of interest to investigate it's influence on bone healing in a fracture healing model with fracture-related infections (FRI) such as osteomyelitis ${ }^{82}$, as might be present during healing of open fractures or pin tract infections which can be present using external fixators ${ }^{85}$. One of the most abundant micro-organisms which is associated with (chronic) osteomyelitis and a high affinity of biofilm formation is Staphylococcus aureus ${ }^{86,87}$ and is furthermore associated with high risks for nonunion development ${ }^{88}$. Relatively recent research observed a reduced in vivo and in vitro $S$. aureus contamination after local delivery of a combination of D-enantiomeric amino acids (D-AA) ${ }^{89}$. In contrast, animal studies and in vitro cell culture studies showed opposing results on stimulation of bone healing using D-AA's ${ }^{90}$, indicating the need for further clarification. 
Adding to the abovementioned future perspectives in animal research (with a possibility for large animal models which resemble the human situation more closely), ideally a large cohort patient study should be conducted supplying enough power to compare consolidating fractures with persisting nonunions within several fracture types. Preferably, a bio-banking system should be set-up to collect blood and bone tissues which can be related to differences in treatmentoutcome of these patients. Next to investigations of the callus and bone tissue in these samples obtained from these patients, the fracture hematoma should not be forgotten. Although already studied since the 1940's, only in recent years it's influence on the fracture healing process ${ }^{91,92}$ - mainly the initial inflammatory phase - is better understood. During systemic inflammatory conditions after trauma, the fracture hematoma might induce chemotaxis of neutrophils with influx into the hematoma hampering the bone healing response ${ }^{93}$. Of interest would be to see if there will be differences visible between the degree of neutrophil influx in the fracture hematoma and the risk of developing a persisting nonunion, which could be underlined by myeloperoxidase expression results found in our nitric oxide deficient mice study and which was linked to decreased callus formation ${ }^{61}$.

\section{Novel findings in this thesis}

- Large amounts of preclinical data exist underlining the influence of the arginine-citrulline-nitric oxide metabolism on fracture healing and nonunion development (chapter 2).

- A femur osteotomy with plate-screw osteosynthesis and additional periosteal injury using electro cauterization leads to delayed union development in mice (chapter 4).

- Periosteal injury induces a delay in healing time of 1 - 2 weeks, which can be extrapolated to $4-6$ weeks in humans (chapter 4 ).

- The new mouse model can provide a continuum between normal bone healing and the development of nonunions (chapter 4).

- Evident nonunion development is observed in nitric oxide synthase deficient mice (chapter 5).

- NOS deficiency additionally leads to deregulated arginine-citrulline-nitric oxide metabolism and prolonged neutrophil influx during fracture healing (chapter 5).

- Distinct differences are observed in the arginine-citrulline-nitric oxide metabolism between samples obtained from patients with successful and unsuccessful nonunion treatment (chapter 6).

- Determination of arginine concentrations and Nos2 expression could be used as predictor for successful treatment of autologous bone grafting in nonunion treatment (Chapter 6). 
- Citrulline treatment results in better post-operative recovery in mice after a femur osteotomy (Chapter 7).

- Moreover, daily oral citrulline treatment improves callus formation and the inflammatory response during bone healing (Chapter 7). 


\section{REFERENCES}

1. van Staa TP, Dennison EM, Leufkens HG, Cooper C. Epidemiology of fractures in England and Wales. Bone 2001; 29(6): 517-22.

2. Alves CJ, Neto E, Sousa DM, et al. Fracture pain-Traveling unknown pathways. Bone 2016; 85: $107-14$.

3. Johnell O, Kanis J. Epidemiology of osteoporotic fractures. Osteoporosis international : a journal established as result of cooperation between the European Foundation for Osteoporosis and the National Osteoporosis Foundation of the USA 2005; 16 Suppl 2: S3-7.

4. Knuth AG, Hallal PC. Temporal trends in physical activity: a systematic review. Journal of physical activity \& health 2009; 6(5): 548-59.

5. Bishop JA, Palanca AA, Bellino MJ, Lowenberg DW. Assessment of compromised fracture healing. J Am Acad Orthop Surg 2012; 20(5): 273-82.

6. Calori GM, Albisetti W, Agus A, Iori S, Tagliabue L. Risk factors contributing to fracture nonunions. Injury 2007; 38 Suppl 2: S11-8.

7. Robinson CM, Court-Brown CM, McQueen MM, Wakefield AE. Estimating the risk of nonunion following nonoperative treatment of a clavicular fracture. J Bone Joint Surg Am 2004; 86-A(7): $1359-65$.

8. Schmidmaier G, Capanna R, Wildemann B, Beque T, Lowenberg D. Bone morphogenetic proteins in critical-size bone defects: what are the options? Injury 2009; 40 Suppl 3: S39-43.

9. Kozin SH. Incidence, mechanism, and natural history of scaphoid fractures. Hand Clin 2001; 17(4): 515-24.

10. Oikawa A, Siragusa M, Quaini F, et al. Diabetes mellitus induces bone marrow microangiopathy. Arteriosclerosis, thrombosis, and vascular biology 2010; 30(3): 498-508.

11. Topping RE, Bolander ME, Balian G. Type X collagen in fracture callus and the effects of experimental diabetes. Clinical orthopaedics and related research 1994; (308): 220-8.

12. Brinker MR, O'Connor DP, Monla YT, Earthman TP. Metabolic and endocrine abnormalities in patients with nonunions. J Orthop Trauma 2007; 21(8): 557-70.

13. Sears BW, Volkmer D, Yong S, et al. Binge alcohol exposure modulates rodent expression of biomarkers of the immunoinflammatory response to orthopaedic trauma. The Journal of bone and joint surgery American volume 2011; 93(8): 739-49.

14. Hastrup SG, Chen X, Bechtold JE, et al. Effect of nicotine and tobacco administration method on the mechanical properties of healing bone following closed fracture. J Orthop Res 2010; 28(9): $1235-9$.

15. Rothem DE, Rothem L, Soudry M, Dahan A, Eliakim R. Nicotine modulates bone metabolismassociated gene expression in osteoblast cells. J Bone Miner Metab 2009; 27(5): 555-61.

16. Day SM, DeHeer DH. Reversal of the detrimental effects of chronic protein malnutrition on long bone fracture healing. J Orthop Trauma 2001; 15(1): 47-53.

17. Long CL, Geiger JW, Richards EW, Akin JM, Blakemore WS. Plasma amino acid concentrations in geriatric control and hip-fracture patients. Am J Clin Nutr 1992; 55(6): 1135-41. 
18. Pollak D, Floman Y, Simkin A, Avinezer A, Freund HR. The effect of protein malnutrition and nutritional support on the mechanical properties of fracture healing in the injured rat. JPEN Journal of parenteral and enteral nutrition 1986; 10(6): 564-7.

19. Cederholm T, Barazzoni R, Austin P, et al. ESPEN guidelines on definitions and terminology of clinical nutrition. Clin Nutr 2017; 36(1): 49-64.

20. Kramer IF, Blokhuis TJ, Verdijk LB, van Loon LJC, Poeze M. Perioperative nutritional supplementation and skeletal muscle mass in older hip-fracture patients. Nutr Rev 2019; 77(4): 254-66.

21. Nowson C, O'Connell S. Protein Requirements and Recommendations for Older People: A Review. Nutrients 2015; 7(8): 6874-99

22. Deutz NE, Matheson EM, Matarese LE, et al. Readmission and mortality in malnourished, older, hospitalized adults treated with a specialized oral nutritional supplement: A randomized clinical trial. Clinical nutrition 2016; 35(1): 18-26.

23. Meesters DM, Wijnands KAP, Brink PRG, Poeze M. Malnutrition and Fracture Healing: Are Specific Deficiencies in Amino Acids Important in Nonunion Development? Nutrients 2018; 10(11).

24. Lackoff AS, Hickling D, Collins PF, Stevenson KJ, Nowicki TA, Bell JJ. The association of malnutrition with falls and harm from falls in hospital inpatients: Findings from a 5-year observational study. J Clin Nurs 2020; 29(3-4): 429-36.

25. Keys A. The residues of malnutrition and starvation. Science 1950; 112(2909): 371-3.

26. Eneroth M, Olsson UB, Thorngren KG. Nutritional supplementation decreases hip fracture-related complications. Clin Orthop Relat Res 2006; 451: 212-7.

27. Patterson BM, Cornell CN, Carbone B, Levine B, Chapman D. Protein depletion and metabolic stress in elderly patients who have a fracture of the hip. J Bone Joint Surg Am 1992; 74(2): 251-60.

28. Fabian E, Gerstorfer I, Thaler HW, Stundner H, Biswas P, Elmadfa I. Nutritional supplementation affects postoperative oxidative stress and duration of hospitalization in patients with hip fracture. Wien Klin Wochenschr 2011; 123(3-4): 88-93.

29. Angele MK, Fitzal F, Smail N, et al. L-arginine attenuates trauma-hemorrhage-induced liver injury. Crit Care Med 2000; 28(9): 3242-8.

30. Poeze M, Bruins MJ, Kessels F, Luiking YC, Lamers WH, Deutz NE. Effects of L-arginine pretreatment on nitric oxide metabolism and hepatosplanchnic perfusion during porcine endotoxemia. $A m J$ Clin Nutr 2011; 93(6): 1237-47.

31. Wijnands KA, Vink H, Briede JJ, et al. Citrulline a More Suitable Substrate than Arginine to Restore NO Production and the Microcirculation during Endotoxemia. PLoS One 2012; 7(5): e37439.

32. Daly JM, Reynolds J, Thom A, et al. Immune and metabolic effects of arginine in the surgical patient. Annals of surgery 1988; 208(4): 512-23.

33. Baldik Y, Talu U, Altinel L, Bilge H, Demiryont M, Aykac-Toker G. Bone healing regulated by nitric oxide: an experimental study in rats. Clin Orthop Relat Res 2002; (404): 343-52.

34. Chae HJ, Park RK, Chung HT, et al. Nitric oxide is a regulator of bone remodelling. J Pharm Pharmacol 1997; 49(9): 897-902.

35. Corbett SA, Hukkanen M, Batten J, McCarthy ID, Polak JM, Hughes SP. Nitric oxide in fracture 
repair. Differential localisation, expression and activity of nitric oxide synthases. J Bone Joint Surg Br 1999; 81(3): 531-7.

36. Corbett SA, McCarthy ID, Batten J, Hukkanen M, Polak JM, Hughes SP. Nitric oxide mediated vasoreactivity during fracture repair. Clin Orthop Relat Res 1999; (365): 247-53.

37. Gerstenfeld LC, Cullinane DM, Barnes GL, Graves DT, Einhorn TA. Fracture healing as a postnatal developmental process: molecular, spatial, and temporal aspects of its regulation. $J$ Cell Biochem 2003; 88(5): 873-84.

38. Schindeler A, McDonald MM, Bokko P, Little DG. Bone remodeling during fracture repair: The cellular picture. Semin Cell Dev Biol 2008; 19(5): 459-66.

39. Garcia P, Histing T, Holstein JH, et al. Rodent animal models of delayed bone healing and nonunion formation: a comprehensive review. European cells \& materials 2013; 26: 1-12; discussion -4 .

40. Histing T, Garcia P, Holstein JH, et al. Small animal bone healing models: standards, tips, and pitfalls results of a consensus meeting. Bone 2011; 49(4): 591-9.

41. Mills LA, Simpson AH. In vivo models of bone repair. J Bone Joint Surg Br 2012; 94(7): 865-74.

42. Holstein JH, Menger MD, Culemann U, Meier C, Pohlemann T. Development of a locking femur nail for mice. Journal of biomechanics 2007; 40(1): 215-9.

43. Bonnarens F, Einhorn TA. Production of a standard closed fracture in laboratory animal bone. Journal of orthopaedic research : official publication of the Orthopaedic Research Society 1984; 2(1): 97-101.

44. Histing T, Holstein JH, Garcia P, et al. Ex vivo analysis of rotational stiffness of different osteosynthesis techniques in mouse femur fracture. Journal of orthopaedic research : official publication of the Orthopaedic Research Society 2009; 27(9): 1152-6.

45. Garcia P, Holstein JH, Histing T, et al. A new technique for internal fixation of femoral fractures in mice: impact of stability on fracture healing. Journal of biomechanics 2008; 41(8): 1689-96.

46. Garcia P, Holstein JH, Maier S, et al. Development of a reliable non-union model in mice. J Surg Res 2008; 147(1): 84-91.

47. Garcia P, Herwerth S, Matthys R, et al. The LockingMouseNail--a new implant for standardized stable osteosynthesis in mice. J Surg Res 2011; 169(2): 220-6.

48. Holstein JH, Matthys R, Histing T, et al. Development of a stable closed femoral fracture model in mice. The Journal of surgical research 2009; 153(1): 71-5.

49. Aro H, Eerola E, Aho AJ. Development of nonunions in the rat fibula after removal of periosteal neural mechanoreceptors. Clinical orthopaedics and related research 1985; (199): 292-9.

50. Matthys R, Perren SM. Internal fixator for use in the mouse. Injury 2009; 40 Suppl 4: S103-9.

51. Grongroft I, Wissing S, Meesters DM, et al. Development of a novel murine delayed secondary fracture healing in vivo model using periosteal cauterization. Arch Orthop Trauma Surg 2019.

52. Dickson KF, Katzman S, Paiement G. The importance of the blood supply in the healing of tibial fractures. Contemporary orthopaedics 1995; 30(6): 489-93.

53. Allen MR, Hock JM, Burr DB. Periosteum: biology, regulation, and response to osteoporosis therapies. Bone 2004; 35(5): 1003-12. 
54. Oetgen ME, Merrell GA, Troiano NW, Horowitz MC, Kacena MA. Development of a femoral nonunion model in the mouse. Injury 2008; 39(10): 1119-26.

55. Raggatt LJ, Wullschleger ME, Alexander KA, et al. Fracture Healing via Periosteal Callus Formation Requires Macrophages for Both Initiation and Progression of Early Endochondral Ossification. The American journal of pathology 2014; 184(12): 3192-204.

56. Claes L, Eckert-Hubner K, Augat P. The effect of mechanical stability on local vascularization and tissue differentiation in callus healing. Journal of orthopaedic research : official publication of the Orthopaedic Research Society 2002; 20(5): 1099-105.

57. Lu C, Miclau T, Hu D, Marcucio RS. Ischemia leads to delayed union during fracture healing: a mouse model. Journal of orthopaedic research : official publication of the Orthopaedic Research Society 2007; 25(1): 51-61

58. Hiltunen A, Vuorio E, Aro HT. A standardized experimental fracture in the mouse tibia. Journal of orthopaedic research : official publication of the Orthopaedic Research Society 1993; 11(2): 305-12.

59. Holstein JH, Garcia P, Histing T, et al. Advances in the establishment of defined mouse models for the study of fracture healing and bone regeneration. J Orthop Trauma 2009; 23(5 Suppl): S31-8.

60. Nunamaker DM. Experimental models of fracture repair. Clinical orthopaedics and related research 1998; (355 Suppl): S56-65

61. Meesters DM, Neubert S, Wijnands KA, et al. Deficiency of inducible and endothelial nitric oxide synthase results in diminished bone formation and delayed union and nonunion development. Bone 2016; 83: 111-8

62. Zhu W, Diwan AD, Lin JH, Murrell GA. Nitric oxide synthase isoforms during fracture healing. $J$ Bone Miner Res 2001; 16(3): 535-40

63. Zhu W, Murrell GA, Lin J, Gardiner EM, Diwan AD. Localization of nitric oxide synthases during fracture healing. J Bone Miner Res 2002; 17(8): 1470-7.

64. Diwan AD, Wang MX, Jang D, Zhu W, Murrell GA. Nitric oxide modulates fracture healing. J Bone Miner Res 2000; 15(2): 342-51.

65. Helfrich MH, Evans DE, Grabowski PS, Pollock JS, Ohshima H, Ralston SH. Expression of nitric oxide synthase isoforms in bone and bone cell cultures. J Bone Miner Res 1997; 12(7): 1108-15.

66. Baldik Y, Diwan AD, Appleyard RC, Fang ZM, Wang Y, Murrell GA. Deletion of iNOS gene impairs mouse fracture healing. Bone 2005; 37(1): 32-6

67. Wijnands KA, Brink PR, Weijers PH, Dejong CH, Poeze M. Impaired fracture healing associated with amino acid disturbances. Am J Clin Nutr 2012; 95(5): 1270-7.

68. Giannoudis PV, Einhorn TA, Schmidmaier G, Marsh D. The diamond concept--open questions. Injury 2008; 39 Suppl 2: S5-8.

69. Giannoudis PV, Gudipati S, Harwood P, Kanakaris NK. Long bone non-unions treated with the diamond concept: a case series of 64 patients. Injury 2015; 46 Suppl 8: S48-54.

70. Ramamurthy C, Cutler L, Nuttall D, Simison AJ, Trail IA, Stanley JK. The factors affecting outcome after non-vascular bone grafting and internal fixation for nonunion of the scaphoid. J Bone Joint Surg Br 2007; 89(5): 627-32. 
71. Calori GM, Phillips M, Jeetle S, Tagliabue L, Giannoudis PV. Classification of non-union: need for a new scoring system? Injury 2008; 39 Suppl 2: S59-63.

72. Zura R, Della Rocca GJ, Mehta S, et al. Treatment of chronic (>1 year) fracture nonunion: heal rate in a cohort of 767 patients treated with low-intensity pulsed ultrasound (LIPUS). Injury 2015; 46(10): 2036-41.

73. Calori GM, Colombo M, Mazza EL, et al. Validation of the Non-Union Scoring System in 300 long bone non-unions. Injury 2014; 45 Suppl 6: S93-7.

74. Flynn NE, Meininger CJ, Haynes TE, Wu G. The metabolic basis of arginine nutrition and pharmacotherapy. Biomed Pharmacother 2002; 56(9): 427-38.

75. Windmueller HG, Spaeth AE. Source and fate of circulating citrulline. Am J Physiol 1981; 241(6): E473-80.

76. Wu G, Morris SM, Jr. Arginine metabolism: nitric oxide and beyond. Biochem J 1998; 336 ( Pt 1): $1-17$.

77. Moinard C, Cynober L. Citrulline: a new player in the control of nitrogen homeostasis. J Nutr 2007; 137(6 Suppl 2): 1621S-5S.

78. Wijnands KA, Hoeksema MA, Meesters DM, et al. Arginase-1 deficiency regulates arginine concentrations and NOS2-mediated NO production during endotoxemia. PLoS One 2014; 9(1): e86135.

79. Vittur F, Lunazzi G, Moro L, et al. A possible role for polyamines in cartilage in the mechanism of calcification. Biochim Biophys Acta 1986; 881(1): 38-45.

80. Claes L, Recknagel S, Ignatius A. Fracture healing under healthy and inflammatory conditions. Nat Rev Rheumatol 2012; 8(3): 133-43.

81. Kdolsky RK, Mohr W, Savidis-Dacho H, et al. The influence of oral L-arginine on fracture healing: an animal study. Wien Klin Wochenschr 2005; 117(19-20): 693-701.

82. Inzana JA, Schwarz EM, Kates SL, Awad HA. A novel murine model of established Staphylococcal bone infection in the presence of a fracture fixation plate to study therapies utilizing antibioticladen spacers after revision surgery. Bone 2015; 72: 128-36.

83. Lu C, Saless N, Hu D, et al. Mechanical stability affects angiogenesis during early fracture healing. J Orthop Trauma 2011; 25(8): 494-9.

84. Wijnands KA, Castermans TM, Hommen MP, Meesters DM, Poeze M. Arginine and Citrulline and the Immune Response in Sepsis. Nutrients 2015; 7(3): 1426-63.

85. Darouiche RO. Treatment of infections associated with surgical implants. The New England journal of medicine 2004; 350(14): 1422-9.

86. Brady RA, Leid JG, Calhoun JH, Costerton JW, Shirtliff ME. Osteomyelitis and the role of biofilms in chronic infection. FEMS immunology and medical microbiology 2008; 52(1): 13-22.

87. Costerton JW, Montanaro L, Arciola CR. Biofilm in implant infections: its production and regulation. Int J Artif Organs 2005; 28(11): 1062-8.

88. Palmer M, Costerton W, Sewecke J, Altman D. Molecular techniques to detect biofilm bacteria in long bone nonunion: a case report. Clinical orthopaedics and related research 2011; 469(11): 3037-42. 
89. Sanchez CJ, Jr., Prieto EM, Krueger CA, et al. Effects of local delivery of D-amino acids from biofilm-dispersive scaffolds on infection in contaminated rat segmental defects. Biomaterials 2013; 34(30): 7533-43.

90. Harmata AJ, Ma Y, Sanchez CJ, et al. D-amino acid inhibits biofilm but not new bone formation in an ovine model. Clinical orthopaedics and related research 2015; 473(12): 3951-61.

91. Grundnes O, Reikeras O. The importance of the hematoma for fracture healing in rats. Acta Orthop Scand 1993; 64(3): 340-2.

92. Park SH, Silva M, Bahk WJ, McKellop H, Lieberman JR. Effect of repeated irrigation and debridement on fracture healing in an animal model. J Orthop Res 2002; 20(6): 1197-204.

93. Bastian OW, Mrozek MH, Raaben M, Leenen LPH, Koenderman L, Blokhuis TJ. Serum from the Human Fracture Hematoma Contains a Potent Inducer of Neutrophil Chemotaxis. Inflammation 2018; 41(3): 1084-92. 



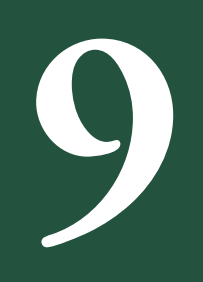

Summary

Nederlandse samenvatting

Impact paragraph

List of abbreviations 


\section{SUMMARY}


Globally, traumatic injuries are one of the major public health burdens and two thirds of all patient visits to an emergency department within the Netherlands are due to accidents, violence or other trauma and result in fractures of one or more bones.

In this thesis, the influence of amino acid metabolism, specifically the argininecitrulline-nitric oxide metabolism is investigated during bone healing and during bone healing difficulties such as delayed union and nonunion development. In the Netherlands, about 250,000 people yearly attend the ermergency department with one or more fractures. Approximately 5-10\% of all these fractures will develop complications during the healing process resulting in delayed union or nonunion. The main hypothesis of this thesis was that the arginine-citrullinenitric oxide metabolism has a crucial influence on an adequate fracture healing process and disturbances in the arginine substrate metabolism play an essential role in development of fracture nonunion, which subsequently can be improved by stimulating the substrate metabolism.

In chapter 1, a background on fracture and nonunion epidemiology is provided, and the (disturbed) healing process of fractures is described from a clinical and biological perspective.

Chapter 2 consists of a literature review starting with a description of the arginine-citrulline-nitric oxide metabolism during normal physiological conditions. Subsequently, the influence of the arginine substrate metabolism is discussed during the complex bone healing process in the clinical setting as well as both the in vivo and in vitro research setting. A disturbed arginine-citrulline-nitric oxide metabolism resulting in altered concentrations of amino acids or expression of important enzymes was found to negatively influence the healing process.

Chapter 3 provides the reader with an overview of the outline of this thesis and the different research objectives which were investigated in this thesis.

In order to be able to investigate the arginine-citrulline-nitric oxide metabolism during bone healing, a reproducible animal model was necessary. In chapter 4, results of the development of a novel mouse model of delayed union without a critically sized defect and with standardized biomechanical conditions were shown. The mice were followed for a period of 42 days, and it was observed that the non-critically sized induced mid-shaft femoral osteotomy which was induced in combination with cauterization of the periosteum resulted in diminished secondary fracture healing 
with significant lower volumes of callus formation. This model enables reliable investigation into the biochemical and molecular biological processes which are ongoing during the bone healing period and additionally can provide an optimal continuum between normal fracture healing and the development of delayed unions and nonunions as a result of the periosteal cauterization.

In chapter $\mathbf{5}$ the results of the first experimental animal study are described. Here it is shown that deletion of the essential nitric oxide synthase enzymes results in an almost complete lack of visible bone healing after 42 days. However, not only the decreased bone volumes on $\mu \mathrm{CT}$ imaging were found. Next to this, the arginine-citrulline-nitric oxide metabolism was found to be deregulated with significant differences in amino acid concentrations both in plasma as well as in femoral tissue. Finally, a disturbed inflammatory reaction was observed in animals with hampered bone healing, with increased and prolonged influx of neutrophils after $28-42$ days of bone healing.

A study into the arginine substrate availability during bone healing complications in humans was presented in chapter 6. In 17 patients which underwent a RIA procedure for autologous bone grafting for atrophic long bone nonunion, reamed bone marrow aspirate was collected and investigated. The patients were subsequently divided regarding the success of the outcome of the RIA procedure. In patients with a successful outcome, arginine and ornithine concentrations were found to be higher when compared to patients with persisting nonunions after the treatment. Additionally, Nos2 expression was higher in all patients that underwent treatment for nonunion when compared to control samples from normally healed fractures. Statistical analysis showed that the determination of arginine concentrations and Nos2 expression levels was subsequently found to be useful as a predictor for successful treatment outcome of autologous bone grafting in nonunion treatment.

Chapter 7 shows the results of our second animal experiment. Here, mice underwent the femur osteotomy which was described before and afterwards received amino acid supplementation for 14 days. Already after two weeks, a significantly increased callus volume was found in treated mice compared to the control group with placebo treated animals. Additionally, gene expression analysis observed decreased expression of inflammatory markers and increased expression of angiogenic and collagen-producing factors in the treated mice, further emphasizing the improved healing process. Although food intake in these mice did not show any difference between the groups, 
citrulline-treated mice showed an increased weight gain over the 14 day period, suggesting a better post-operative recovery.

In the final chapter 8 the obtained results in all studies presented in this thesis are combined and discussed with regards to all available recent literature. Also, it summarized on the main findings and it elaborates on the possibilities for the direction future research in the field of the arginine-citrulline-nitric oxide metabolism during bone healing and nonunion development.

In summary, this dissertation shows that the arginine substrate metabolism is of importance during fracture repair as genetic or pharmacologic inhibition results in inadequate healing responses with disturbed inflammation and decreased callus formation, whereas stimulation of the metabolism increases bone formation and results in a faster bone healing with beneficial biomolecular and biochemical responses. 
NEDERLANDSE SAMENVATTING 
Traumatische verwondingen zijn wereldwijd een van de grootste gezondheidsproblemen. Ongeveer twee derde van alle patiëntbezoeken aan de spoedeisende hulp van de Nederlandse ziekenhuizen vindt plaats vanwege ongevallen, geweld of andere fysieke trauma's, waarbij een groot deel resulteert in een of meerdere botfracturen.

In deze dissertatie is de invloed van het aminozuurmetabolisme, specifiek het arginine-citrulline-stikstofmonoxide (NO) metabolisme onderzocht zowel tijdens normale botgenezing als tijdens gecompliceerde botgenezing zoals vertraagde genezing en de vorming van non-union (gestopte, onvolledige botgenezing van een fractuur, ook wel eens pseudoarthrose genoemd). De belangrijkste in deze thesis onderzochte hypothese was dat het arginine-citrulline-NO-metabolisme van cruciale invloed is op een adequaat botgenezingsproces en dat verstoringen van het arginine substraatmetabolisme een essentiële rol spelen in de ontwikkeling van non-unions. Verbetering van het substraatmetabolisme zou vervolgens de fractuurgenezing bevorderen.

In hoofdstuk 1 wordt een achtergrond geschetst over het voorkomen van botfracturen en non-unions met de hierbij horende risicofactoren, en wordt tevens het (verstoorde) genezingsproces en beschreven vanuit zowel een klinisch and een biochemisch en biomedisch perspectief.

Hoofdstuk 2 bevat een overzicht van de bekende literatuur omtrent eiwitten en aminozuren in relatie met botgenezing, en begint met een beschrijving van het arginine-citrulline-NO-metabolisme tijdens normale fysiologische omstandigheden. Vervolgens wordt het arginine substraatmetabolisme besproken tijdens het botgenezingsproces vanuit zowel de humane (klinische) context als vanuit de experimentele in vitro en in vivo invalshoek. Een verstoring van het arginine-citrulline-NO-metabolisme resulterend in veranderde concentraties van aminozuren en veranderde genetische expressie van enzymen is gerelateerd aan een verslechterde botgenezing.

Hoofdstuk 3 toont de lezer een overzicht van de structuur van deze dissertatie en bespreekt de verschillende onderzoeksdoelen welke bestudeerd zijn.

Om het arginine-citrulline-NO-metabolisme te onderzoeken tijdens botgenezing is een betrouwbaar en reproduceerbaar diermodel gewenst. In hoofdstuk $\mathbf{4}$ zijn de resultaten van de ontwikkeling van een nieuw muismodel met vertraagde botgenezing getoond, zonder dat gebruik wordt gemaakt van een groot segmentaal defect maar waarbij het periosteum wordt beschadigd 
middels electrocauterizatie. De studie laat gestandaardiseerde biomechanische resultaten liet zien, onder andere in de resultaten van de onderzochte buigstijfheid van de femurs. De muizen werden gedurende een periode van 42 dagen opgevolgd en er werd gezien dat een midschacht femurosteotomie in combinatie met elektrocauterizatie van het periosteum resulteerde in verminderde secundaire botgenezing met significant verlaagde volumes van callus (botnieuwvorming) en een vertraging van de genezing van 14 tot 28 dagen. Het ontwikkelde model zorgt voor een betrouwbare onderzoeksmogelijkheid naar de biochemische en moleculaire aspecten tijdens de botgenezing en kan tevens een optimaal continuüm verzorgen tussen normale fractuurgenezing en de non-union-ontwikkeling als gevolg van het induceren van periosteale schade bij een onderbreking in de botstructuur zoals ook tijdens fracturen optreedt.

In hoofdstuk 5 zijn de resultaten beschreven van de eerste dierstudie waarin het eerder beschreven diermodel is gebruikt. Het blokkeren van de essentiële stikstofmonoxide-synthase enzymen resulteert in een bijna volledige afwezigheid van botgenezing na 42 dagen. Naast de, via micro-CT, gevonden sterk verlaagde callusvolumes in deze muizen, werd er ook een ontregeld argininecitrulline-stikstofmonoxide metabolisme ontdekt met significante afwijkende aminozuurconcentraties in zowel bloed als botweefsel. Tenslotte was er ook een verstoorde inflammatoire reactie te zien in de dieren met verstoorde botgenezing, waarbij een verhoogde en, in tijd verlengde, influx van neutrofielen nog zichtbaar was in de periode na 28 tot 42 dagen botgenezing.

Gerelateerd aan dit experimentele werk is de arginine substraatbeschikbaarheid tijdens verstoorde botgenezing bij patiënten beschreven in hoofdstuk 6. Van 17 patiënten die een RIA-procedure ondergingen voor autologe beenmergtransplantatie voor atrofische non-union van de lange pijpbeenderen werd reamed beenmergaspiraat verzameld en onderzocht. De verkregen resultaten werden vervolgens verdeeld in twee groepen afhankelijk van het uiteindelijke succes van de behandeling die zij hebben ondergaan. In patiënten waarbij de RIA behandeling zorgde voor een succesvolle botgenezing, waren arginine en ornithine concentraties hoger wanneer dit vergeleken werd met patiënten met persisterende non-unions na behandeling. Tevens was Nos2 expressie hoger in alle patiënten die deze behandeling voor non-unions ondergingen wanneer dit werd vergeleken met controle weefsels die waren verzameld bij patiënten met een normale fractuurgenezing. Statistisch onderzoek toonde aan dat de determinatie van arginine concentraties en het expressieniveau van Nos2 gebruikt kan worden als voorspelling voor een succesvolle behandeling van autologe beenmergtransplantatie voor pseudoarthroses. 
In hoofdstuk 7 worden de resultaten gepresenteerd van dierexperimenteel onderzoek naar de bevordering van fractuurgenezing. Muizen ondergingen wederom een femurosteotomie en kregen vervolgens gedurende 14 dagen extra suppletie van aminozuren. Na deze twee weken werden significant verhoogde callusvolumes gevonden in vergelijking met de groep die een placebo behandeling onderging. Tevens liet genexpressie-analyse verlaagde niveaus van inflammatoire markers zien waarbij juist de niveaus van angiogene (vaatnieuwvorming) en collageen (bindweefselvorming) factoren verhoogd waren. Hoewel de voedselinname in deze groepen vergelijkbaar was, lieten muizen die suppletie van citrulline kregen een verhoogde gewichtstoename zien, mogelijk wijzend richting een betere postoperatief herstel.

In het laatste hoofdstuk 8 zijn alle verkregen resultaten uit de eerder getoonde studies gecombineerd en bediscussieerd aan de hand met alle recente beschikbare literatuur. Tenslotte worden hier de belangrijkste conclusies van ons onderzoek samengevat en worden de mogelijkheden voor toekomstig onderzoek in het onderwerp verder verdiept.

Samenvattend laat deze dissertatie zien dat het arginine substraatmetabolisme van belang is tijdens fractuurgenezing omdat genetische of farmacologische remming resulteert in een inadequaat genezingsproces met verstoorde inflammatie en verminderde callusvorming, en waar stimulatie van het metabolisme resulteert in verhoogde botvorming en versnelde genezing met voordelige biomoleculaire en biochemische reacties. 


\section{IMPACT PARAGRAPH}




\section{Main objectives and results}

The main goal of the research described in this dissertation is to investigate the role of amino acid metabolism, specifically the arginine-citrulline-nitric oxide metabolism, on the fracture healing process and the development of complications during bone healing, such as delayed healing and nonunion development. This metabolism can influence the fracture healing process via three distinct ways. Firstly, through stimulation of the metabolism, amino acids are formed from proteins to act as precursors for collagen, one of the main constituents of bone tissue. Secondly, one of the most relevant enzymes in this metabolism is the inducible nitric oxide synthase. This enzyme influences the regulation of inflammation, which is especially important during the first phase of the bone healing cascade. Disturbances during this phase often lead to complications later on during the healing process. Finally, the angiogenic reactivity is stimulated via several enzymes, and is of importance for optimal vascularization of the newly formed bone.

The most important findings described in the thesis are firstly that the combination of a femur osteotomy with periosteal injury induced by electrocauterization result in a highly reproducible and reliable mouse model for investigating bone healing and bone healing difficulties. Subsequently, this model was used to investigate a disrupted amino acid metabolism which was shown to lead to an almost complete absence of bone healing, and which is concurrent with an adverse effect on the inflammatory reaction during bone healing. Contrariwise, when stimulating the amino acid metabolism by additional oral supplementation, the normal physiologic bone healing process shortened by approximately 30\% and a stimulated collagen formation and a beneficial inflammatory response were observed.

Finally, in patients with long bone nonunions who underwent autologous bone grafting treatment, the levels of several amino acids and genes related to the arginine-citrulline-nitric oxide metabolism obtained during the surgical procedure, were found to be able to act as a predictor for defining the success of treatment outcome.

\section{Scientific, ethical and societal impact}

In the last decades, a wide range of different murine fracture healing and delayed union and nonunion models were developed. The model described earlier in this thesis resulted in better controlled biomedical condition as compared to other fixation techniques and the obtained tissues could be used for biomechanical, biochemical, genetic, radiographic and histological analysis. Recently, a new generation of comparable plates is developed in which polyether-ether-ketone is coated in titanium. Using these plates, every animal can then be monitored multiple times and without the need of euthanasia for data collection. This way, 
research can be even more in compliance with the $3 \mathrm{R}$ principle of reduction, replacement and refinement in animal testing and can drastically lower the amount of animals needed for experimentation.

Mainly elderly patients with (hip) fractures suffer from a substantial loss of skeletal muscle mass (and subsequent function). Citrulline supplementation stimulates skeletal muscle and total body weight in both experimental and clinical studies. The increase in body weight in our amino acid supplementation study, after the reduced weight loss during the first postoperative days indicates an advantageous recovery and rehabilitation period. This taken together with the $30 \%$ shortened healing period until the bone parts are united may indicate that citrulline can improve postoperative recovery in the frail elderly population.

In literature, the treatment for fracture nonunions is structured in a pentagonal concept describing the five key stones for adequate treatment: local mechanical stability, active bone cells (osteoblast and osteoclasts), the presence of an adequate scaffold, sufficient vascularity at the fracture site and lastly the presence of growth factors necessary for callus formation. The findings in our study strongly suggest that if proven in the clinical setting, the nutritional status of the patients should also be taken into account, thus transforming the treatment into a hexagonal concept. To be able to finalize this concept, studies into the amino acid metabolism should thus be conducted in patients that are prone to developing an impaired fracture healing such as multitrauma patients or in the frail elderly with an already marginal nutritional status.

The results described in this thesis can have a huge clinical potential and are of great interest for clinicians, dieticians, industrial partners and mainly the patients, but also for fellow reseachers working in the field of bone healing metabolism. Therefore the research in this thesis is already (partially) published in peer-reviewed scientific journals such as Bone, the Archives of Orthopaedic and Trauma Surgery and European Cells and Materials, and have also been presented at several distinguished national and international conferences such as the European Society of Tissue Regeneration in Orthopaedics and Traumatology and the International Society for Fracture Repair.

Within the Maastricht University Medical Center, the importance of an adequate non-union treatment is recognized. Recently, a multidisciplinary outpatient clinic has started in which the findings from several clinical and translational studies is put to practice by trauma-, orthopaedic and plastic surgeons in the treatment of patients with nonunions. 


\section{LIST OF ABBREVIATIONS}


AEC

ANOVA

Arg1

Asl

Ass

BMI

BMP

CDNA

$\mathrm{CO}_{2}$

$\mathrm{Col}$

$\mathrm{Cxcl} 2$

DEPC

Dkk1

DM

EDTA

FDA

FRI

$H \& E$

HPLC

HRP

IFN- $\gamma$

IGF-1

IL-1, 6, 8

IVC

L-NAME

$\mu-\mathrm{CT}$

$\mathrm{MPO}$

mRNA

$\mathrm{NaAc}$

$\mathrm{NaCl}$

$\mathrm{NaOH}$

$\mathrm{NF}-\kappa \mathrm{B}$

NO

Nos1

Nos2

Nos3

NSAIDs

NUSS

OP-1

OTC 3-amino-9-ethylcarbazole

analysis of variance

arginase 1

argininosuccinate lyase

argininosuccinate synthase

body mass index

bone morphogenetic protein

copy desoxyribonucleic acid

carbon dioxide

collagen

chemokine (C-X-C motif) ligand 2

diethylpyrocarbonate

Dickkopf-related protein 1

diabetes mellitus

ethylenediaminetetraacetic acid

United States Food and Drug Administration

fracture-related infection

haematoxylin \& eosin

high performance liquid chromatography

horseradish peroxidase

interferon gamma

insulin-like growth factor 1

interleukin-1, -6 or -8

individually ventilated cages

$\mathrm{N}(\omega)$-nitro-L-arginine methyl ester

micro computed tomography

myeloperoxidase

messenger ribonucleic acid

sodium acetate

sodiumchloride

sodium hydroxide

nuclear factor kappa-light-chain-enhancer of activated B cells

nitric oxide

neuronal nitric oxide synthase (also nNOS)

inducible nitric oxide synthase (also iNOS)

endothelial nitric oxide synthase (also eNOS)

nonsteroidal anti-inflammatory drugs

nonunion scoring scale

osteogenic protein 1 (BMP7)

ornithine transcarbamylase 
PCR polymerase chain reaction

PEEK poly-ether-ether ketone

RANKL receptor activator of nuclear factor kappa-B ligand

RIA reamer-irrigator-aspirator

ROI region of interest

Scl sclerostin

SEM standard error of the mean

TGF- $\beta$ transforming growth factor beta

TNF- $\alpha \quad$ tumor necrosis factor alpha

VEGF vascular endothelial growth factor 



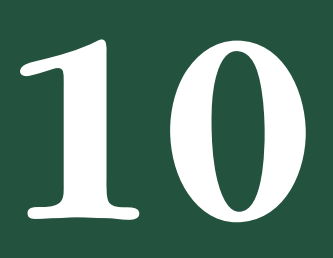

\section{Dankwoord \\ List of publications \\ Curriculum vitae}




\section{DANKWOORD}


In april 2009 begon ik op de afdeling Algemene Heelkunde aan mijn afstudeerstage voor het HBO. Op dat moment kon ik nog niet bevoelen dat dit jaren later zou kunnen resulteren in een proefschrift. Veel personen hebben in meer of mindere mate een belangrijke rol gespeeld in de totstandkoming van dit boekje.

Allereerst mijn promotor, dagelijkse supervisor en begeleider, prof. dr. Poeze. Beste Martijn, dank voor de mogelijkheid die je mij geboden hebt om dit onderzoek binnen de traumachirurgie te beginnen en tevens voor alle uitleg over de klinische achtergronden die mij als niet-medicus in den beginne allemaal vreemd waren. Zo nu en dan betrapte ik mezelf erop dat ik het klinische deel veel interessanter vond dan het biologische deel in het lab. Dank ook voor de talloze koffiemomenten en alle verdere ondersteuning bij de interpretatie van onderzoeksresultaten, je statistische kennis en het corrigeren van mijn manuscripten binnen korte (of soms langere) tijd. Uiteraard werd dit zo nu en dan afgewisseld met relax momenten, zoals de caipirinha's in een obscure Brazil Bar in München, tapas en ritjes in geblindeerde taxibusjes in Malaga en jaarlijkse teppanyaki-avonden in Amsterdam. Alles bij elkaar heeft ervoor gezorgd dat ik met veel plezier terugdenk aan de aangename tijd waarin de onderzoeken zijn uitgevoerd!

Dan mijn copromotor, dr. Wijnands, beste Nina, je was letterlijk mijn kleinste begeleider tijdens mijn promotie, maar zeker niet de minste! Er is denk ik niemand van wie ik in de afgelopen jaren meer geleerd heb over het uitvoeren van experimenteel onderzoek, het onderhouden van allerlei samenwerkingen en de begeleiding van studenten (vaak met het nodige gezucht). Jouw mate van orde houden in alle digitale bestanden is ongeëvenaard en vaak goed van pas gekomen als ik weer eens een document nodig had. Dank ook voor alle gezellige middagen en avonden bij jou en Ramon! Het was altijd weer een verrassing of er een huisvarken langswandelde, een terror-kip of een Slayer-hond!

Beste prof. dr. Brink, officieel heeft u geen rol in mijn promotieteam, maar zeker in het begin van mijn periode was u erg betrokken bij het onderzoek en heeft $\mathrm{u}$ de basis gelegd voor de samenwerking die geresulteerd heeft in het gebruikte diermodel in deze thesis. Dank ook voor alle nuttige feedback op abstracts, posters, manuscripten en presentaties. De kwaliteit werd er altijd - vaak door maar een aanpassing van een of twee woorden - veel beter door.

Dear members of the thesis assessment committee, prof. dr. T.J.M. Welting, prof. dr. G. Schmidmaier, prof. dr. M.J. Stoddart, prof. dr. M. van Griensven and dr. T.J. Blokhuis, thank you for assessing the quality of this thesis and your willingness to participate (online or on-site) in the PhD ceremony. 
Twee personen hebben een grote invloed gehad op mij gehad tijdens het uitvoeren van het onderzoek, vandaar dat zij op de dag van de verdediging achter mij zullen staan als paranimfen.

Als eerste dr. Dello, beste Simon, de combinatie van jouw relaxedheid maar tevens ook gedrevenheid is een ideale eigenschap voor een onderzoeker! Dank voor je inspiratie die je mij hebt gegeven om een "eigen projectje" te starten, maar daarnaast natuurlijk ook voor alle ontspanning tijdens onder andere het Maastrichtse ambtenarencarnaval, de diverse labuitjes en de daarop aansluitende late avonden (en dus indirect ook voor de katers de dag erna).

Dr. Hannemann, beste Pascal, zonder jouw steun had dit boekje er heel anders uitgezien. Als een ware Yoda had je tijdens elke kop koffie wel een advies paraat over onderzoek en onderwijs en de betere Bourgogne-wijnen. Na allerlei referenties naar cultfilms en culthelden uit de wielersport eindigden de gesprekken meestal in flauwe grappen die wij alleen zelf begrepen. Dank ook voor alle gezellige culinaire genotsmomenten van Maastricht tot aan het Lago Maggiore. Hopelijk kunnen we dit nog een lange tijd voortzetten!

Ook alle andere huidige en in het verleden in Maastricht werkzame traumachirurgen, Jan Verbruggen, Paul Breedveld, Taco Blokhuis, Guido Stollenwerck, Hester Graeler en Jan ten Bosch, dank voor de mogelijkheden om de verschillende klinische besprekingen bij te wonen en de leerzame momenten die ik altijd heb ervaren tijdens het verzamelen van mijn weefsels in de operatiekamer. Manuela Heinrichs, door jouw aanwezigheid verlopen alle administratieve en organisatorische zaken binnen deze groep een stuk vloeiender! Dank voor alle ondersteuning, ook als ik een afspraak met Martijn weer eens drie keer moest verplaatsen.

Dear dr. Stephan Zeiter, thank you for the collaboration which resulted in two of the chapters presented in this thesis, and for your patience when teaching us the animal model in Maastricht. Hopefully I will be able to visit Davos in the future!

De micro-CT metingen werden verricht bij de afdeling Biomedical Engineering van de Technische Universiteit Eindhoven. Dank aan Bert van Rietbergen voor uitleg omtrent de metingen en de kwantificatie van onze resultaten.

De medewerkers van het $\mathbf{C P V}$ ben ik dank verschuldigd voor hun assistentie bij het verzorgen van onze proefdieren en het voorbereiden van de operatieruimten. 
Voor het onderzoek beschreven in deze dissertatie werd gebruikt gemaakt van de laboratoria van de afdeling algemene heelkunde, eerst onder leiding van prof. dr. Wim Buurman en vervolgens prof. dr. Steven Olde Damink, beiden dank ik voor hun (soms kritische) commentaren en uitdagende vragen tijdens de wekelijkse presentaties en werkbesprekingen. Hetzelfde geldt ook voor de vaste stafleden van het lab: Kaatje Lenaerts, Sander Rensen en Frank Schaap.

De belangrijkste personen in een laboratorium zijn de analisten. Toen ik in 2009 op de afdeling begon was Tim Wolfs mijn stage- en afstudeerbegeleider. In deze periode heb jij mij de fijne kneepjes van het vak geleerd, PCR's, blotten, histologie, ELISA's, bacteriekweek en zelfs het maken van BSA. Dank hiervoor, dit heeft mij een gedegen technische achtergrond opgeleverd en veel van de methodes (en nog meer de flauwe practical jokes) gebruik ik nog steeds tot op de dag van vandaag. Veel dank ook dat je plaats neemt in de corona om mij ook tijdens deze verdediging ongetwijfeld stevig te zullen ondervragen over mijn kennis.

Beste Hans van Eijk, toen ik na mijn stage als medewerker begon was jij mijn eerste kamergenoot en later hebben we nog 2 jaar intensief samengewerkt op het metabool lab. Jouw kennis over massaspectrometrie en chromatografie (en veel andere onderwerpen) is in mijn ogen haast oneindig. De twee belangrijkste zaken die ik altijd zal onthouden: "chromatografie is een kunst" en "met een wattenstaafje en wat geduld is alles schoon te krijgen". Mo Hadfoune, de vele overdenkingen tijdens de lunch, koffiepauzes en maandagochtenden eindigden vaak met de conclusie dan de Berbers de grondleggers zijn van een schier oneindig aantal culinaire lekkernijen en wetenschappelijke ontdekkingen. Jammer dat je jezelf nog niet tot de ontdekker van een goed coach-van-het-jaar team of tourpoule team kunt bekronen. Voor het verbouwen van kantoormuren kan men altijd terecht bij Bas Boonen! Dank voor de allereerste introductie op het lab met de legendarische HEL-ELISA, maar meer nog voor alle gezelligheid - om 15.00 uur op het terras zitten kan best! Annemarie van Bijnen, het rustpunt tussen de mannen in het lab en een ware ELISA queen. Als ik ooit problemen ondervind bij deze techniek dan weet ik je te vinden!

Collegae promovendi die uit dezelfde periode in het lab en kamergenoten: Dirk Schellekens, Brabantse maïs-specialist, hard werken als kamergenoten, maar ook genoeg tijd voor allerlei randzaken die in meer of mindere mate significant bijdragen aan onderzoek doen. German Friday zou verplicht moeten worden! Kostan Reisinger, een korte omschrijving: Kostanios, Hans Teeuwen imitator, AFC specialist, the raging bull, schrijftalent, dank voor alle gezelligheid op onze kamer tijdens de laatste maanden van je promotie! Ruben Vogels (zouden mensen last van ons hebben gehad op de kamer in de middenlob?) en Inca Hundscheid 
(carnavalsvierster bij uitstek!), regelmatig hebben wij elkaar na onze avonturen op het lab ook nog gezien en gesproken. Laten we hopen dat we dit ook nog kunnen voortzetten nu we niet meer binnen 20 minuten rijden van elkaar wonen. Claire Leenarts, ik kan hier heel veel zaken benoemen, maar ik houd het erbij dat je jouw ezels geen rotte appels moet voeren!

Met veel (oud)collegae op het heelkunde lab heb ik nooit direct samengewerkt, maar veel hebben wel gezorgd voor nieuwe kennis en leermomentjes tijdens de jaren dat ik op het lab heb rondgelopen: Romy Aarnoutse, Martijn Arts, JoyceManyi Bakia, Kevin van Barneveld, Kirsten van der Beek, Rianne Beckers, Johanne Bloemen, Jacqueline van den Bos, Anne-Claire Bosmans, Maartje van den Broek, Joep Derikx, David van Dijk, Rob van Gassel, Irma Geenen, Freek Gillissen, Briete Goorts, Joep Grootjans, Jacco de Haan, Luc Heijnen, Cathelijne Heymans, Leontine van den Hil, Cathy van Himbeeck, Caroline Hodin, Pieter Hoogland, Kirsten Huntjens, Renske Janssen, Dennis Japink, Mechteld de Jong, Charlotte de Jonge, Audrey Jongen (pindakaas?), Mirjam Kip, Kiran Koelfat, Jasper Kox, Irene Fleur Kramer, Ralph Kurstjens, Toine Lodewick, Tiara Lopez Penha, Tim Lubbers, Milou Martens, Kim van Mierlo, Elwin Mommers, Liliane Mpabanzi, Martine Moossdorff (is het nog steeds Mr. Big?), Evelien Neis (blondie), Thiemo van Nijnatten, Loes Nijssen, Givan Paulus, Selwyn van Rijn, Yvonne Roebroek, Lori van Roozendaal, RobertJan Schipper, Rutger Schols, Marc Schreinemacher, Filip Segers, Tim van Smaalen, Livia Smits (goed gereedschap hangt inderdaad onder een afdakje!), Maarten Snoeijs, Zita Soons, Rob Strijkers, Geertje Thuijls, Rianne Vaes, Froukje Verdam, Iris Vermeulen Windsant, Ruben Visschers, Luuk de Wert (jammer dat je PSV supporter bent), Kim van Wijck, Victor van Woerden, Mark de Wolf, Edgar Wong-Lun-Hing, Sofia Xanthoulea en Junfang Zhao.

Het jarenlang Engelse artikels schrijven zorgt ervoor dat de Nederlandse grammatica in de Nederlandse samenvatting van dit proefschrift soms nogal verengelst was. Dank aan Thom Laming om dit kritisch te bekijken en waar nodig te corrigeren.

Ook veel stagiaires hebben meegewerkt aan de experimenten en onderzoeken in dit proefschrift. Zonder de inzet van Lauren Kusters, Marc van den Beemt, Frans Heyer, Amber Geomini, Levi Smeets, Vera Schriebl en Jeroen Smit zouden diverse taken veel langer geduurd hebben. Ook dank aan de studentassistenten Merle Geerds, Clint Boymans, Alexandra Leenders, Michael Houben, Oscar van Katwijk, Romy Verkaik en Julia Bels die volkomen vrijwillig hebben bijgedragen aan het soms saaie database-onderzoek. Deze data wordt vaak niet genoemd maar leidt vaak wel tot nieuwe inzichten in de eerder 
verkregen resultaten en nieuwe ideeën voor vervolgonderzoek.

Naast het werk is ontspanning met vrienden natuurlijk nog veel belangrijker. Tim en Christian, we kennen elkaar al sinds de middelbare school, besloten alle drie naar het $\mathrm{HBO}$ te gaan en werden vervolgens ook carpool-buddies die met gevaar voor eigen leven elke dag naar Maastricht reden. De hilarische belevenissen onderweg zal ik hier maar niet beschrijven. Dank voor alle gezelligheid, uiteraard ook tijdens alle feestjes, barbecues en andere samenkomsten. Onze dames hebben ons regelmatig met enige verwondering bekeken, maar zulke vreemde dingen hebben we toch nooit gedaan? Daarnaast hebben we samen met Bart, Karel en Chris al jaren ons maandelijkse kaartavondje dat voor de nodige ontspanning zorgt. Hopelijk kunnen we dit nog lange tijd doorzetten!

Mijn ouders wil ik danken voor hun onvoorwaardelijke steun die zij mij altijd hebben gegeven voor alles wat ik ooit heb gedaan en bereikt op privé en professioneel gebied. Hopelijk gaat het lekenpraatje tijdens mijn presentatie enige verduidelijking geven over alle 'moeilijke' dingen die ik in het laboratorium heb uitgevoerd.

Een bekend adagium binnen de traumachirurgie is dat er niet te veel koosnaampjes in een dankwoord verschijnen en dat het opdragen van een proefschrift aan je levenspartner eigenlijk een veel te omslachtige manier is om je genegenheid te tonen. Als biomedisch onderzoeker binnen dit vakgebied wil ik me hier dan ook graag bij aansluiten. Lieve Anne (knoesj), dank voor alle steun in de afgelopen jaren, mijn boekje is nu ook eindelijk klaar! Samen met onze vrolijke spartel Julia kunnen we nu nog meer gaan genieten van alle mooie momenten die we in de rest van ons leven met zijn 3en nog zullen meemaken. Ik hou van jullie! 


\section{LIST OF PUBLICATIONS}




\section{Publications}

1. Wijnands KA, Hoeksema MA, Meesters DM, van den Akker NMS, Molin DMG, Briedé JJ, Ghosh M, Köhler SE, van Zandvoort MAMJ, de Winther MPJ, Buurman WA, Lamers WH, Poeze M. Arginase-1 deficiency regulates arginine concentrations and Nos2-mediated NO production during endotoxemia. PLoS One. 2014 Jan 21;9(1):e86135.

2. van Wijck K, Wijnands KA, Meesters DM, Boonen B, van Loon LJ, Buurman WA, Dejong $\mathrm{CH}$, Lenaerts K, Poeze M. L-citrulline improves splanchnic perfusion and reduces gut injury during exercise. Med Sci Sports Exerc. 2014 Nov;46(11):2039-46.

3. Wijnands KA, Castermans TM, Hommen MP, Meesters DM, Poeze M. Arginine and citrulline and the immune response in sepsis. Nutrients. 2015 Feb 18;7(3):1426-63.

4. Wijnands KA, Meesters DM, van Barneveld KW, Visschers RG, Briedé JJ, Vandendriessche B, van Eijk HM, Bessems BA, van den Hoven N, von Wintersdorff CJ, Brouckaert P, Bouvy ND, Lamers WH, Cauwels A, Poeze M. Citrulline supplementation improves organ perfusion and arginine availability under conditions with enhanced arginase activity. Nutrients. 2015 Jun 29;7(7):5217-38.

5. Meesters DM, Neubert S, Wijnands KAP, Heyer FL, Zeiter S, Ito K, Brink PRG, Poeze M. Deficiency of inducible and endothelial nitric oxide synthase results in diminished bone formation and delayed union and nonunion development. Bone. 2016 Feb;83:111-118.

6. Meesters DM, Wijnands KAP, Brink PRG, Poeze M. Malnutrition and fracture bealing: are specific deficiencies in amino acids important in nonunion development? Nutrients. 2018 Oct 31;10(11):1597.

7. Wong J, Lenaerts K, Meesters DM, Olde Damink SWM, van Eijk HMH, Vilar E, Farrington K. Acute haemodynamic changes during haemodialysis do not exacerbate gut hyperpermeability. Biosci Rep. 2019 Apr 12;39(4):BSR20181704.

8. Gröngröft I, Wissing S, Meesters DM, Poeze M, Matthys-Mark R, Ito K, Zeiter S. Development of a novel murine delayed secondary fracture bealing in vivo model using periosteal cauterization. Arch Orthop Trauma Surg. 2019 Dec;139(12):1743-1753.

9. Meesters DM, Hannemann PF, van Eijk HM, Schriebl VT, Brink PR, Poeze M, Wijnands KA. Enhancement of fracture bealing after citrulline supplementation in mice. Eur Cell Mater. 2020 Mar 20;39:183-192.

10. Shiri-Sverdlov R, dos Reis IM, Oligschlaeger Y, Hendrikx T, Meesters DM, Vanclooster A, Vanhoutvin N, Koek GH, Westerterp M, Binder CJ, Cassiman $\mathrm{D}$, Houben $\mathrm{T}$. The influence of a conjugated pneumococcal vaccination on plasma antibody levels against oxidized low-density lipoprotein in metabolic 
disease patients: a single-arm pilot clinical trial. Antioxidants (Basel). 2021 Jan 18;10(1):129.

11. Meesters DM, Wijnands KAP, van Eijk HMH, Hofman M, Hildebrand F, Verbruggen JPAM, Brink PRG, Poeze M. Arginine availability in reamed intramedullary aspirate as predictor of outcome in nonunion healing. Submitted.

12. Reintam Blaser A, Padar M, Mändul M, Elke G, Engel C, Fischer K, Giabicani M, Gold T, Hess B, Hiesmayr M, Jakob SM, Loudet CI, Meesters DM, Mongkolpun W, Paugam-Burtz C, Poeze M, Preiser JC, Renberg M, Rooijackers O, Tamme K, Wernerman J, Starkopf J. Development of the gastrointestinal dysfunction score (GIDS) for critically ill patients - a prospective multicenter observational study (iSOFA study). Submitted.

13. Wijnands KAP, Meesters DM, Vandendriessche B, Briedé J, van Eijk HMH, Brouckaert P, Cauwels A, Poeze M. Microcirculatory function during endotoxemia: a functional citrulline-arginine-NO-patbway and Nos3 complex is essential to maintain the microcirculation. In preparation.

\section{Oral presentations}

1. Wijnands KAP, Briedé JJ, Vink H, Meesters DM, Buurman WA, Lamers WH, Poeze M. Citrulline suppletie in sepsis verbeterd de microcirculatie via de endotheliale NOS geïnduceerde NO produktie. XXIII SEOHS 2010, Rotterdam, The Netherlands.

2. Wijnands KAP, Vink H, Meesters DM, Köhler ES, Buurman WA, Lamers WH, Poeze M. Detrimental effects of arginase-1 deficiency on the microcirculation and NO production during sepsis. European Society of Intensive Care Medicine (ESICM) 2011, Berlin, Germany.

3. Wijnands KAP, van den Akker NMS, Ghosh M, Meesters DM, Briedé JJ, Köhler ES, van Zandvoort MAMJ, Lamers WH, Molin DMG, Poeze M. Endothelialspecific argininosuccinate-synthase knock-out mice have impaired arginine de novo synthesis, NO production and microcirculation during endotoxemia. European Society of Intensive Care Medicine (ESICM) 2012, Lisbon, Portugal.

4. van den Hoven N, Wijnands KAP, Meesters DM, Poeze M. Citrulline improves the microcirculation in an arginine deficiency state. Mosa Conference 2013, Maastricht, The Netherlands.

5. Wijnands KAP, Hoeksema MA, Meesters DM, van den Akker NMS, Molin DGM, Briedé JJ, Ghosh M, Köhler ES, van Zandvoort MAMJ, de Winther MPJ, Buurman WA, Lamers WH, Poeze M. Arginase-1 activity regulates arginine concentrations and macrophage NOS2-mediated NO production during endotoxemia. XXVI SEOHS 2013, Maastricht, The Netherlands.

6. Wijnands KAP, Meesters DM, Vandendriessche B, Briedé JJ, Bessems 
BAFM, van Eijk HMH, Brouckaert P, Buurman WA, Cauwels A, Poeze M. A functional citrulline-arginine-NO-pathway and NOS3 complex is essential to maintain microcirculatory function during endotoxemia. XXVI SEOHS 2013, Maastricht, The Netherlands.

7. Meesters DM, Wijnands KAP, Brink PRG, Poeze M. Aminozuurmetabolisme tijdens fractuurgenezing en nonunion. Wetenschapsavond Frailty Fractures en Botkwaliteit, 2015, Maastricht, The Netherlands.

8. Meesters DM, Wijnands KAP, Brink PRG, Poeze M, Zeiter S. Ontwikkeling van een nieuw delayed union en nonunion muismodel. XXVIII SEOHS 2015, Leiden, The Netherlands.

9. Meesters DM, Wijnands KAP, van Eijk HMH, Verbruggen JPAM, Brink PRG, Poeze M. Arginine availability in reamed intramedullary aspirate-influences on fracture bealing and nonunion development. $15^{\text {th }}$ Biennial Conference of the International Society for Fracture Repair 2016, Munich, Germany.

10. Meesters DM, Hannemann PFW, van Eijk HMH, Schriebl VTJ, Brink PRG, Poeze M. Bevordering van fractuurgenezing middels citrulline suppletie in muizen. XXIX SEOHS 2016, Utrecht, The Netherlands. (best abstract nominee)

11. Meesters DM, Wijnands KAP, van Eijk HMH, Boymans C, Brink PRG, Poeze M. Arginine beschikbaarbeid in beenmerg als indicatie voor succesvolle reamer-irrigator-aspirator behandeling van nonunions. NVT Traumadagen 2017, Amsterdam, The Netherlands.

12. Ding L, Goossens GH, Oligschläger Y, Houben T, Meesters DM, Blaak EE, Shiri-Sverdlov R. Plasma cathepsin D activity is negatively associated with bepatic insulin sensitivity in overweight and obese patients. Dutch Liver Retreat 2019, Spier, The Netherlands.

13. Meesters DM, Hannemann PFW, van Eijk HMH, Schriebl VTJ, Brink PRG, Poeze M, Wijnands KAP. Stimulation of fracture bealing using citrulline supplementation in mice. $5^{\text {th }}$ European Society of Tissue Regeneration in Orthopaedics and Traumatology (ESTROT) Congress 2019, Malaga, Spain.

\section{(Invited faculty member)}

14. Padar M, Starkopf J, Forbes A, Wernerman J, Rooijackers O, Jakob SM, Hiesmayr M, Gold T, Poeze M, Meesters DM, Reintam Blaser A. Plasma citrulline dynamics in intensive care medicine. European Society of Intensive Care Medicine (ESICM) Lives 2019, Berlin, Germany.

\section{Poster presentations}

1. Wijnands KAP, Briedé JJ, Vink H, Meesters DM, Köhler SE, Buurman WA, Lamers WH, Poeze M. Detrimental effects of arginase-1 deficiency on the microcirculation and NO production during sepsis. NUTRIM Research 
Symposium 2011, Maastricht, The Netherlands.

2. Meesters DM, Wijnands KAP, Brink PRG, Poeze M. Arginine and NO metabolism during fracture repair and nonunion development. VLAG PhD week 2012, Baarlo, The Netherlands.

3. Heyer FL, Meesters DM, Wijnands KAP, Zeiter S, Ito K, Brink PRG, Poeze M. Arginase-1 and nitric oxide synthase isoforms in fracture bealing and nonunion. Mosa Conference 2013, Maastricht, The Netherlands.

4. Meesters DM, Wijnands KAP, Heyer FL, Boymans C, Zeiter S, Ito K, Brink PRG, Poeze M. NOS-depletion inhibits fracture bealing and enhances nonunion development in mice. XXVI SEOHS 2013, Maastricht, The Netherlands.

5. Meesters DM, Wijnands KAP, Heyer FL, Zeiter S, Ito K, Brink PRG, Poeze M. Alterations in arginine metabolism influence fracture bealing and nonunion development. NUTRIM Research Symposium 2013, Maastricht, The Netherlands.

6. Wijnands KAP, Meesters DM, Vandendriessche B, Briedé JJ, Bessems BAFM, van Eijk HMH, Brouckaert P, Buurman WA, Cauwels A, Poeze M. A functional citrulline-arginine-NO pathway and NOS3 complex is essential to maintain microcirculatory function during endotoxemia. European Society of Intensive Care Medicine (ESICM) 2014, Barcelona, Spain.

7. Meesters DM, Wijnands KAP, Zeiter S, Brink PRG, Poeze M. Disturbed arginine-NO metabolism inbibits callus formation resulting in delayed-and nonunion. NUTRIM Research Symposium 2014, Maastricht, The Netherlands.

8. Meesters DM, Neubert S, Wijnands KAP, Zeiter S, Ito K, Brink PRG, Poeze M. Nitric oxide synthase deficiency inbibits callus formation resulting in nonunion development. 4th ECTS-IBMS 2015, Rotterdam, The Netherlands.

\section{(travel grant award)}

9. Meesters DM, Wijnands KAP, Brink PRG, Zeiter S, Poeze M. Development of a novel murine delayed union and nonunion model of fracture bealing. NUTRIM Research Symposium 2015, Maastricht, The Netherlands.

10. Meesters DM, Smit J, Wijnands KAP, Verbruggen JPAM, Brink PRG, Poeze M. Evaluatie van de reamer-irrigator-aspirator (RIA) procedure tijdens de bebandeling van nonunions. NVT Traumadagen 2016, Amsterdam, The Netherlands.

11. Wong J, Lenaerts K, Meesters DM, van Eijk HMH, Vilar E, Farrington K. Measurement of gut permeability in haemodialysis patients. British Renal Society Conference 2017, Nottingham, UK.

12. Bitorina AV, Jeurissen MLJ, Houben $\mathrm{T}$, Meesters DM, Walenbergh SMA, Oligschläger Y, Plat J, Lütjohann D, Romano A, Shiri-Sverdlov R. Cellular gender identity - the inflammatory effects of 27-bydroxycholesterol are sex dependent. NUTRIM Research Symposium 2017, Maastricht, The Netherlands. 
13. Blokhuis TJ, Meesters DM, Ma KF, Poeze M. Fractuurhematoom bevat RNA voor BMP-7 en NF-кB. NVT Traumadagen 2017, Amsterdam, The Netherlands.

14. Schriebl VTJ, Meesters DM, Hannemann PFW, van Eijk HMH, Brink PRG, Wijnands KAP, Poeze M. Arginine-NO metabolism during fracture healing. Mosa Conference 2018, Maastricht, The Netherlands.

15. Ding L, Oligschläger Y, Houben T, Verwer B, Meesters DM, Tushuizen ME, Shiri-Sverdlov R. Cathepsin D activity, rather than levels, correlated with the development of type 2 diabetes mellitus and metabolic syndrome. NUTRIM Research Symposium 2018, Maastricht, The Netherlands.

16. Padar M, Starkopf J, Forbes A, Wernerman J, Rooijackers O, Jakob SM, Hiesmayr M, Gold T, Poeze M, Meesters DM, Reintam Blaser A. Plasma I-FABP dynamics in intensive care medicine. European Society of Intensive Care Medicine (ESICM) Lives 2019, Berlin, Germany. 


\section{CURRICULUM VITAE}


Dennis Meesters was born in Heinsberg, Germany, on December 28 $8^{\text {th }}$ 1987. After high school (VWO, Nature and Health, Rombouts College, Brunssum) he studied Biology and Medical Laboratory Research at Zuyd University of applied sciences in Heerlen. His first research internship was performed at the Department of Internal Medicine, division of Clinical and Experimental Immunology of Maastricht University under supervision of Dr. J.G.M.C. Damoiseaux. He obtained his Bachelor of Applied Science degree for

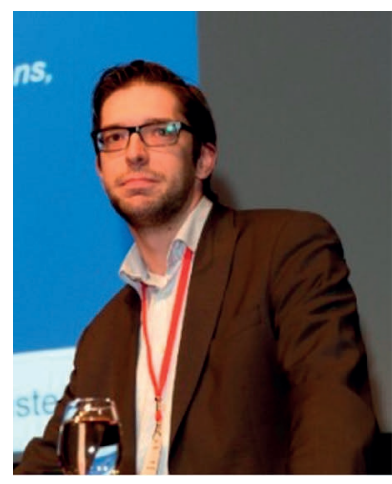
the thesis describing his work at the Department of General Surgery (Maastricht University), in which he investigated the production and purification of Histagged I-FABP antibodies (supervision: Dr. T.G.A.M. Wolfs and Prof. Dr. W.A. Buurman). Afterwards he started working as a research technician at this lab in 2009, focussing on the sepsis research project together with Dr. K.A.P. Wijnands and Prof. Dr. M. Poeze. In February 2012, he started his PhD project into the influence of amino acids on fracture healing and nonunion development at the department of Trauma Surgery under supervision of Prof. Dr. M. Poeze and Dr. K.A.P. Wijnands, resulting in the current thesis. The different studies were presented at several national and international conferences, including the $4^{\text {th }}$ joint meeting of the European Calcified Tissue Society and the International Bone \& Mineral Society meeting in Rotterdam (2015), the 15th biennial conference of the International Society for Fracture Repair (Munich, 2016), the Dutch Trauma Days (Amsterdam, 2017) and the $5^{\text {th }}$ European Society for Tissue Regeneration in Orthopaedics and Traumatology congress (Malaga, 2019). During the last part of this project, he also worked as a research assistant at the metabolic ward of the laboratory of General Surgery (Dr. H.M.H. van Eijk and Prof. Dr. S.W.M. Olde Damink). Since July 2017, he works as a senior research assistant and lab manager at the Department of Genetics and Cell Biology (Prof. Dr. R. Shiri-Sverdlov).

Dennis happily lives together with Anne Dirks in Maastricht. In October 2019 their daughter Julia was born. 


